\title{
Exploiting Ligand Conformation in the Selective Inhibition of Non-Ribosomal Peptide Synthetase Amino Acid Adenylation with Designed Macrocyclic Small Molecules
}

\author{
Justin S. Cisar, ${ }^{\dagger, \star}$ Julian A. Ferreras, ${ }^{\S}$ Rajesh K. Soni,${ }^{\S}$ Luis E. N. Quadri, ${ }^{* \dagger,}{ }^{\S}$ Derek S. Tan*, ${ }^{*, *}$ \\ ${ }^{\dagger}$ Tri-Institutional Training Program in Chemical Biology, \\ ${ }^{\ddagger}$ Tri-Institutional Research Program and Molecular Pharmacology \& Chemistry Program, \\ Memorial Sloan-Kettering Cancer Center, 1275 York Avenue, Box 422, New York, New York 10021 and \\ ${ }^{\S}$ Department of Microbiology \& Immunology and Molecular Biology Program \\ Weill Medical College of Cornell University, 1300 York Avenue, Box 62, New York, New York 10021
}

\section{Supporting Information}
A. Structural analysis of adenylation enzymes and
S1 aminoacyl-tRNA synthetases with bound ligands
B. Conserved binding interactions of NRP synthetase
S3 amino acid adenylation domains with ligands
C. Materials and methods
S6
D. Synthesis of linear aminoacyl-AMS compounds (1a-e)
S7
E. Synthesis of cyclo $^{8 \mathrm{C}_{2} \beta}$-Ala-AMS macrocycles $(\mathbf{2 a}, \mathbf{2 c})$
S10
F. Synthesis of cyclo $^{8 \mathrm{C}_{3} \beta}$-Ala-AMS macrocycles $(\mathbf{2 b}, \mathbf{2 d})$
S19
G. Synthesis of cyclo $^{8 \mathrm{C}_{3} \beta}$-propionyl-AMS macrocycle (2e)
S28
H. HMWP2 inhibition assay
S31
I. In vitro translation assay
S33
J. NMR spectra for macrocycles $\mathbf{2 a - e}$
S34

\section{A. STRUCTURAL ANALYSIS OF ADENYLATION ENZYMES AND AMINOACYL-tRNA SYNTHETASES WITH BOUND LIGANDS}

As described briefly in the manuscript, examination of all available protein crystal structures of adenylation enzymes (and closely related enzymes) and aminoacyl-tRNA synthetases in complex with ligands revealed distinct protein folds and distinct binding conformations. In the structures of a phenylalanine adenylation domain (PheA), a 2,3-dihydroxybenzoate adenylation enzyme (DhbE), luciferase, and a long chain fatty acyl-CoA synthetase (LC-FACS), the ligands are all bound in overall cisoid conformations (Figure S1a-d). In all 17 available structures of ligandbound aminoacyl-tRNA synthetases (not shown), the ligands are bound in overall extended or transoid conformations. ${ }^{1}$ This general conformation is observed for both Class I and Class II aminoacyl-tRNA synthetases (Figure S1e-f).

\footnotetext{
${ }^{1}$ For a review, see: Ibba, M.; Soll, D. Annu. Rev. Biochem. 2000, 69, 617-650.
} 
A)

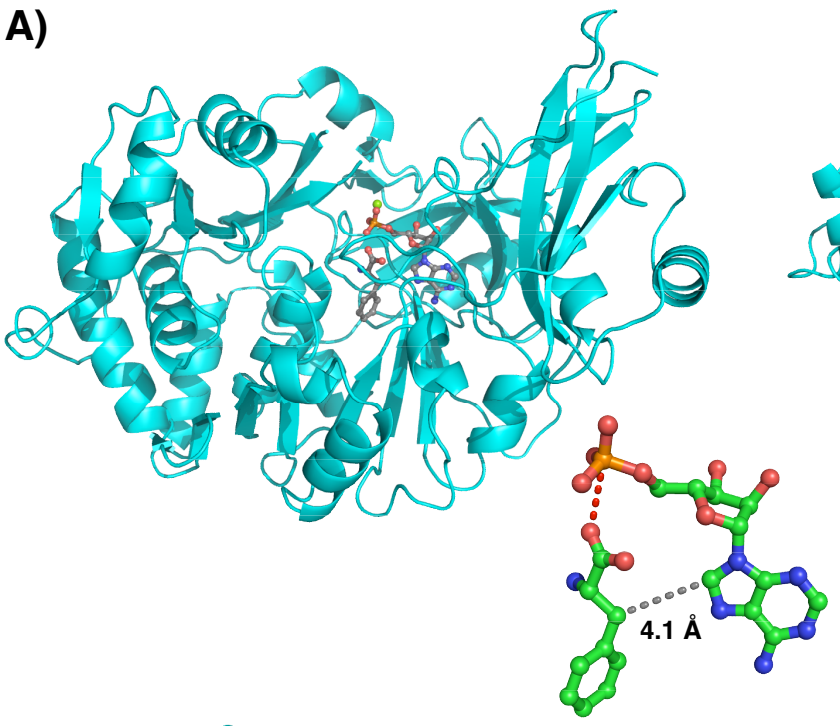

B)

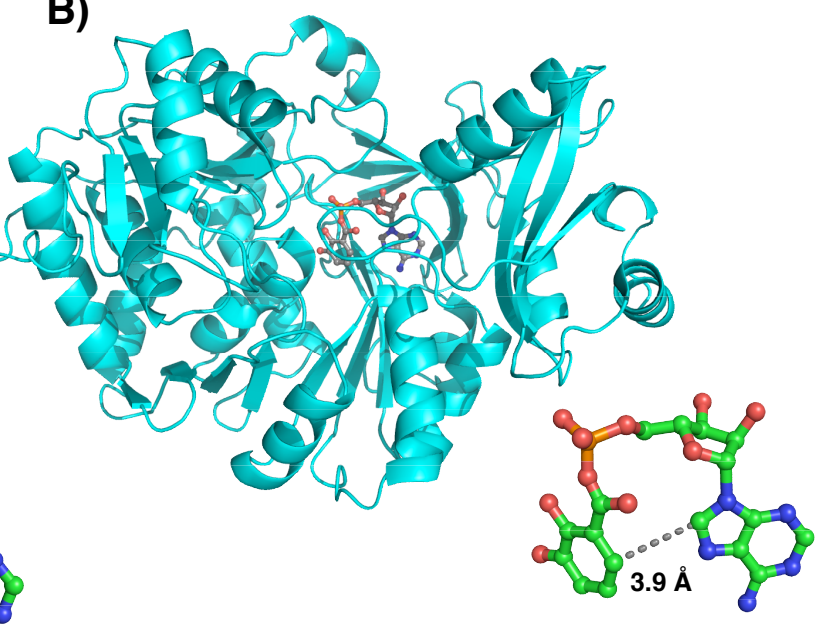

C)
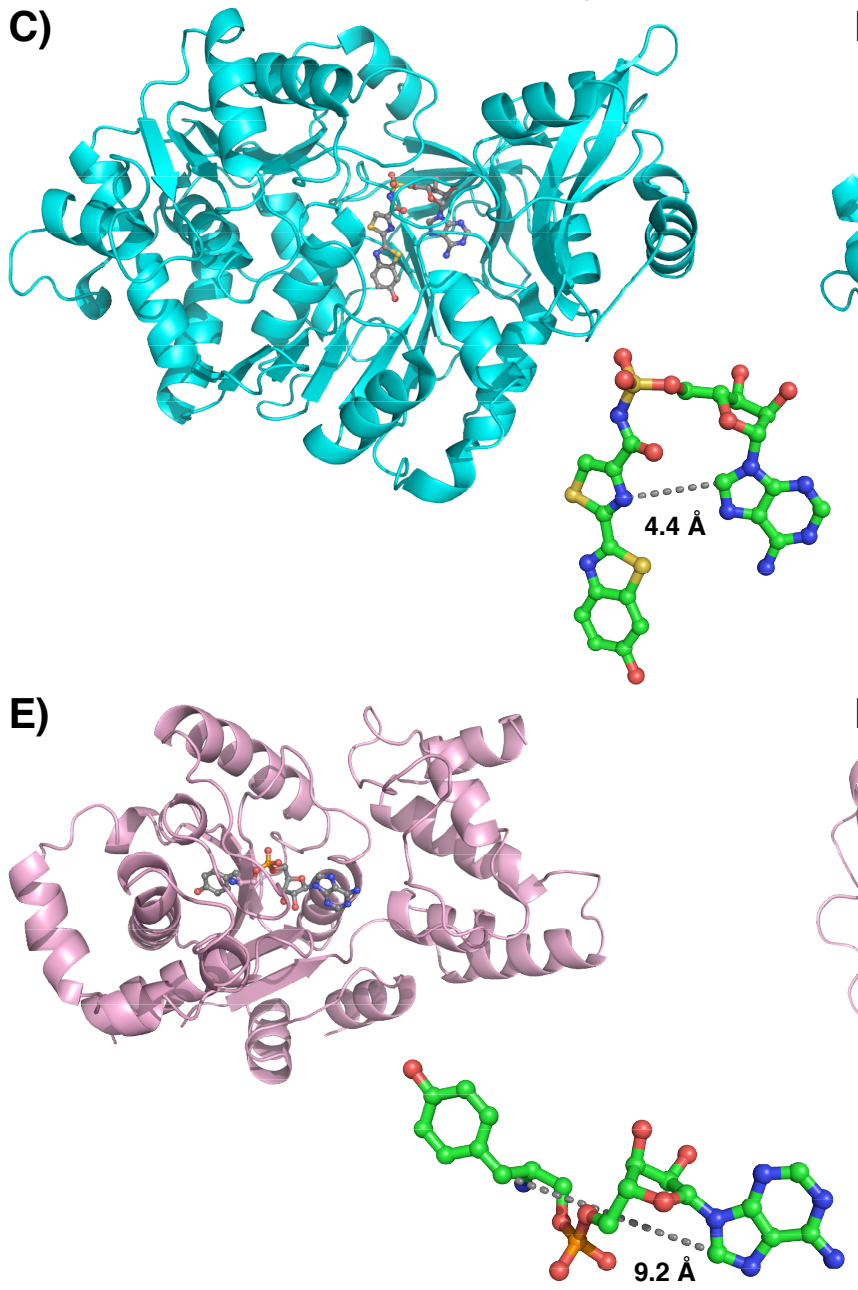

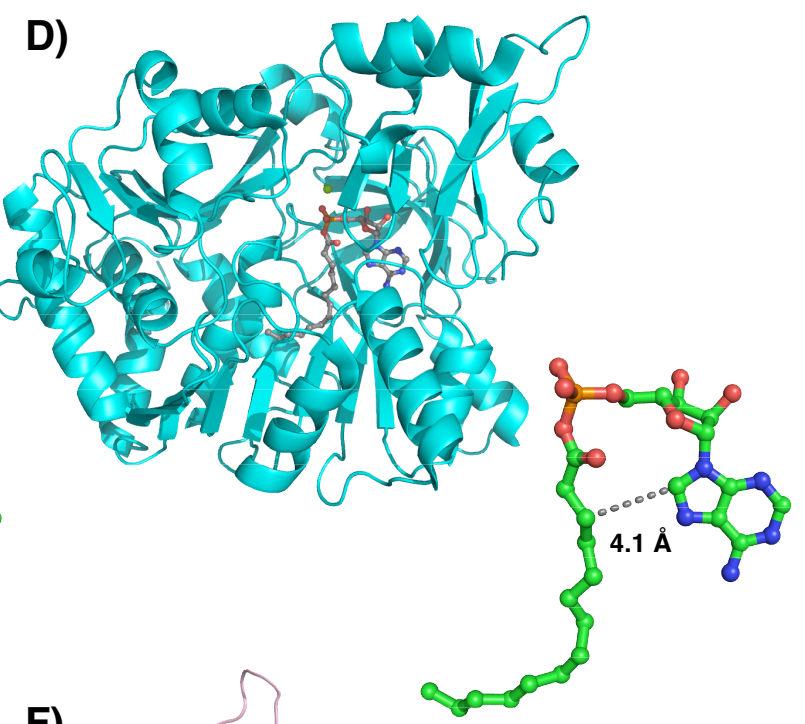

Figure S1. Protein crystal structures of various related adenylation enzymes (cyan: A-D) and two aminoacyl-tRNA synthetases representing both Class I and Class II enzymes (pink: E,F). Bound ligand conformations are shown (insets) with measured distances between adenine $C 8$ and the $\beta$-position of the acyl group indicated. (A) Phenylalanine adenylation domain (PheA) from Bacillis brevis gramicidin 
S synthetase 1 (GrsA) with bound phenylalanine and AMP (PDB 1AMU). ${ }^{2}$ (B) 2,3-Dihydroxybenzoate adenylation enzyme (DhbE) from Bacillus subtilis bacillibactin synthetase with bound 2,3-dihydroxybenzoyl-AMP (PDB 1MDB). ${ }^{3}$ (C) Luciferase from Luciola cruciata with bound luciferyl-AMS, a luciferylAMP sulfamate analog (PDB 2D1S). ${ }^{4}$ (D) Long chain fatty acyl-CoA synthetase (LC-FACS) from Thermus thermophilus HB8 with bound myristoyl-AMP (PDB 1V26). ${ }^{5}$ (E) Tyrosyl-tRNA synthetase (TyrRS) from Bacillus stearothermophilus, a Class I enzyme, with bound tyrosinyl-AMP, a carbonylreduced analog of Tyr-AMP (PDB 3TS1). ${ }^{6}$ (F) Phenylalanyl-tRNA synthetase (PheRS) from Thermus thermophilus, a Class II enzyme, with bound phenylalaninyl-AMP, a carbonyl-reduced analog of Phe-AMP (PDB 1B7Y). ${ }^{7}$

\section{B. CONSERVED BINDING INTERACTIONS OF NRP SYNTHETASE AMINO ACID ADENYLATION DOMAINS WITH LIGANDS}

The single available crystal structure of an amino acid adenylation domain, the phenylalanine adenylation domain (PheA) of gramicidin S synthetase 1 (GrsA), was analyzed in greater detail for comparison to the cysteine adenylation domain of yersiniabactin synthetase HMWP2 that was used in the studies described in this manuscript. PheA binding residues were identified based on the original report of Conti et $a l^{2}$ and examination of the corresponding crystal structure (PDB 1AMU) (Figure S2a). Sequence alignments were then carried out for 39 amino acid adenylation domains selected primarily from siderophore synthetases (Table S1). Amino acid adenylation domain sequences were designated using annotated AMP-binding regions with the addition of $70 C$-terminal residues to include the $C$-terminal lobe of the proteins, based on the crystal structure of PheA. Sequence alignments were then performed using LaserGene MegAlign with the ClustalW method and default parameters. Sequences were manually trimmed or extended and realigned reiteratively to match the $N$ - and $C$-termini of PheA. Overall sequence identity in the final alignment averaged 35.0\% (range 18.5-99.8\%). Putative key binding residues were then identified for the cysteine adenylation domain of HMWP2 and other related cysteine, serine, and threonine adenylation domains based on the PheA structural analysis (Figure S2b). As expected from the previous report of Stachelhaus et al., ${ }^{8}$ key binding residues were highly conserved, with the exception of several residues that likely dictate amino acid specificity. Interestingly, Lys517 (PheA numbering) was not completely conserved despite its important role in general binding to acyl-AMP ligands. Notably, however, a conformational change observed in the structure of LC-FACS (Figure S1d) may account for this, with an alternative, completely conserved lysine (Lys434 in PheA) possibly fulfilling this role in some proteins.

\footnotetext{
${ }^{2}$ Conti, E.; Stachelhaus, T.; Marahiel, M. A.; Brick, P. EMBO J. 1997, 16, 4174-4183.

${ }^{3}$ May, J. J.; Kessler, N.; Marahiel, M. A.; Stubbs, M. T. Proc. Natl. Acad. Sci. U.S.A. 2002, 99, $12120-12125$.

${ }^{4}$ Nakatsu, T.; Ichiyama, S.; Hiratake, J.; Saldanha, A.; Kobashi, N.; Sakata, K.; Kato, H. Nature 2006, 440, 372376.

${ }^{5}$ Hisanaga, Y. et al. J. Biol. Chem. 2004, 279, 31717-31726.

${ }^{6}$ Brick, P.; Bhat, T. N.; Blow, D. M. J. Mol. Biol. 1989, 208, 83-98.

${ }^{7}$ Reshetnikova, L.; Moor, N.; Lavrik, O.; Vassylyev, D. G. J. Mol. Biol. 1999, 287, 555-568.

${ }^{8}$ Stachelhaus, T.; Mootz, H. D.; Marahiel, M. A. Chem. Biol. 1999, 6, 493-505.
} 


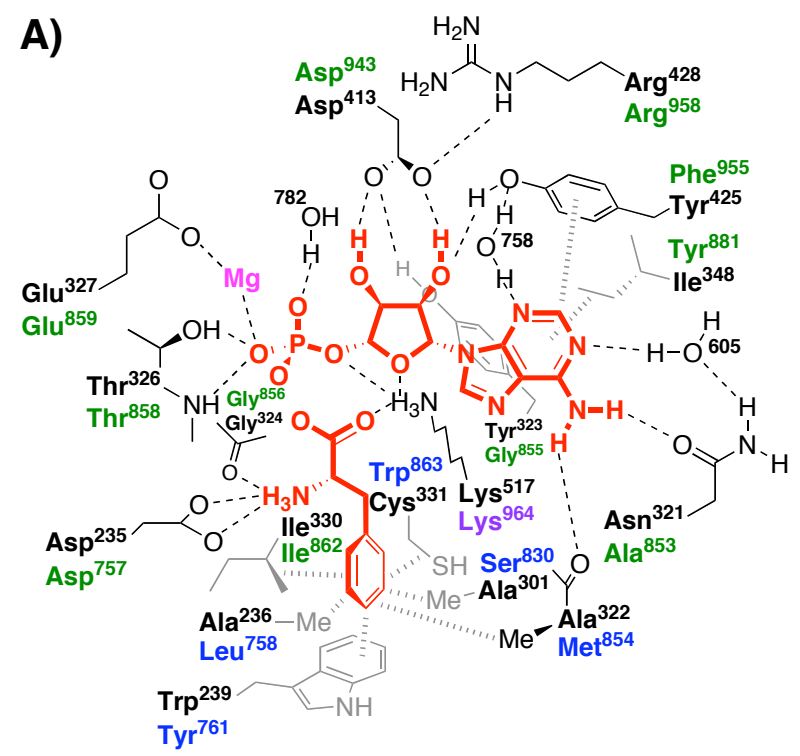

B)

\begin{tabular}{|c|c|c|}
\hline \multicolumn{2}{|c|}{ GrsA residue: } & \multirow{2}{*}{ 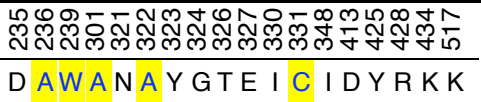 } \\
\hline GrsA & Phe & \\
\hline PvdL & Tyr & D A E GNA Y GTE VM I D Y R KK \\
\hline HMWP2 & Cys & D LY SAMGGTE I WYDF R K L* \\
\hline PchE & Cys & D LF S V LGGTE I WYDFR KG* \\
\hline PchF & Cys & D L Y S S LGGTE I WYDFRKA* \\
\hline AngR & Cys & DLYSAMGGTE I WYDFRKK \\
\hline \multirow[t]{2}{*}{ Pvdl } & $\operatorname{Ser}^{4}$ & DVWS NLYGTE I D I D Y R KK \\
\hline & $\operatorname{Ser}^{6}$ & D VWSNLYGTE I D I D Y R KK \\
\hline EntF & Ser & D VWSNLYGTEVD I D Y R KK \\
\hline MbtB & Ser & DMLGGLGGTEVHYDFR KK \\
\hline \multirow[t]{2}{*}{ PvdD } & $\operatorname{Thr}^{-10}$ & D FWGNMYGTE VH I DYR KK \\
\hline & $\operatorname{Thr}^{11}$ & D FWGNMYGTEVH I DYR KK \\
\hline DhbF & Thr & D FWGNMYGTE V H I D Y R K K \\
\hline BasA & Thr & D MF GS LGGTE I WYDFR KK \\
\hline VibF & Thr & D MF GS LGGTE I WYDFR KK \\
\hline
\end{tabular}

Figure S2. Amino acid adenylation domain ligand binding interactions. (A) Schematic representation of binding interactions between the phenylalanine adenylation domain (PheA) of gramicidin S synthetase 1 (GrsA) and the ligands phenylalanine and AMP (PDB 1AMU). ${ }^{2}$ Residues within $4.0 \AA$ of the ligand are displayed, along with putative hydrogen bonding (---) and hydrophobic ( $\left.\ldots . \cdot)^{\prime}\right)$ interactions. GrsA residues (black) and corresponding yersiniabactin synthetase HMWP2 cysteine adenylation domain residues (green, blue, purple) are indicated, with putative amino acid specificity determining residues highlighted (blue). PheA Lys517 is proposed to be replaced by a distinct conserved lysine residue in HMWP2 (purple, Lys964; corresponding to Lys434 in PheA). (B) Putative key binding residues of several cysteine, serine, and threonine adenylation domains, including the cysteine adenylation domain from HMWP2, based on sequence alignments. 
Table S1. Amino acid adenylation domains used in sequence alignments.

\begin{tabular}{|c|c|c|c|c|c|}
\hline Organism & Synthetase & Protein & GenBank & Domain & Residues \\
\hline Yersinia pestis & yersiniabactin & HMWP2 & NP_669706 & Cys & $593-1067$ \\
\hline Bacillis brevis & gramicidin S & $\begin{array}{l}\text { GrsA } \\
\text { GrsB }\end{array}$ & $\begin{array}{l}\text { P0C062 } \\
\text { P0C064 }\end{array}$ & $\begin{array}{l}\text { Phe } \\
\text { Pro } \\
\text { Val } \\
\text { Orn } \\
\text { Leu }\end{array}$ & $\begin{array}{l}66-530 \\
495-963 \\
1533-1998 \\
2569-3043 \\
3615-4081\end{array}$ \\
\hline \multirow[t]{5}{*}{ Pseudomonas aeruginosa PAO1 } & pyochelin & $\begin{array}{l}\text { PchE } \\
\text { PchF }\end{array}$ & $\begin{array}{l}\text { NP_252916 } \\
\text { NP_252915 }\end{array}$ & $\begin{array}{l}\text { Cys }^{1} \\
\text { Cys }^{2}\end{array}$ & $\begin{array}{l}581-1049 \\
541-1014\end{array}$ \\
\hline & pyoverdine $\mathrm{A}$ & PvdL & NP_251114 & $\begin{array}{l}\text { Glu } \\
\text { Tyr } \\
\text { Dab }\end{array}$ & $\begin{array}{l}1155-1629 \\
2213-2696 \\
3740-4237\end{array}$ \\
\hline & & Pvdl & AAX16297 & $\begin{array}{l}\text { Ser }^{1} \\
\text { Arg } \\
\text { Ser }^{2} \\
\text { hfOrn }\end{array}$ & $\begin{array}{l}544-1011 \\
2021-2492 \\
3071-3538 \\
4570-5053\end{array}$ \\
\hline & & PvdJ & NP_251090 & $\begin{array}{l}\text { Lys } \\
\text { hfOrn }\end{array}$ & \\
\hline & & PvdD & NP_251089 & $\begin{array}{l}\text { Thr }^{1} \\
\text { Thr }^{2}\end{array}$ & $\begin{array}{l}529-1012 \\
1590-2073\end{array}$ \\
\hline Mycobacterium tuberculosis H37Rv & mycobactin $\mathrm{T}$ & $\begin{array}{l}\text { MbtB } \\
\text { MbtE } \\
\text { MbtF }\end{array}$ & $\begin{array}{l}\text { NP_216899 } \\
\text { NP_216896 } \\
\text { NP_216895 }\end{array}$ & $\begin{array}{l}\text { Ser } \\
\text { Lys }^{1} \\
\text { Lys }^{2}\end{array}$ & $\begin{array}{l}579-1044 \\
466-930 \\
498-956\end{array}$ \\
\hline Lystonella/Vibrio anguillarum & anguibactin & AngR & NP_943553 & Cys & $480-955$ \\
\hline Acinetobacter baumannii & acinetobactin & BasA & BAC87897 & Thr & $48-518$ \\
\hline Vibrio cholerae & vibriobactin & VibF & AAG00566 & Thr & $922-1397$ \\
\hline Bacillis subtilis & bacillibactin & DhbF & AAD56240 & $\begin{array}{l}\text { Gly } \\
\text { Thr }\end{array}$ & $\begin{array}{l}485-955 \\
1541-2030\end{array}$ \\
\hline Escherichia coli K12 & enterobactin & EntF & $N P \_415118$ & Ser & $482-965$ \\
\hline Tolypocladium niveum & cyclosporin & CssA & CAA82227 & $\begin{array}{l}\text { Ala-1 } \\
\text { Leu-2 } \\
\text { Leu-3 } \\
\text { Val-4 } \\
\text { Bmt-5 } \\
\text { Abu-6 } \\
\text { Gly-7 } \\
\text { Leu-8 } \\
\text { Val-9 } \\
\text { Leu-10 } \\
\text { Ala-11 }\end{array}$ & $\begin{array}{l}513-994 \\
1599-2076 \\
3096-3568 \\
4582-5059 \\
6075-6553 \\
7572-8049 \\
8633-9109 \\
10127-10601 \\
11616-12092 \\
12696-13166 \\
14194-14667\end{array}$ \\
\hline
\end{tabular}




\section{Materials and Methods}

Reagents were obtained from Aldrich Chemical (www.sigma-aldrich.com) or Acros Organics (www.fishersci.com) and used without further purification. Optima grade solvents were obtained from Fisher Scientific (www.fishersci.com), degassed with Ar, and purified on a solvent drying system as described ${ }^{9}$ unless otherwise indicated. Triethylamine $\left(\mathrm{Et}_{3} \mathrm{~N}\right)$, diisopropylethylamine (DIPEA), and diisopropylamine $\left(i-\mathrm{Pr}_{2} \mathrm{NH}\right)$ were distilled from $\mathrm{CaH}$ under $\mathrm{N}_{2}$. Reactions were performed in flame-dried glassware under positive Ar pressure with magnetic stirring. Cold baths were generated as follows: $0{ }^{\circ} \mathrm{C}$, wet ice/water; $-10{ }^{\circ} \mathrm{C}$, dry ice/chloroform; $-78^{\circ} \mathrm{C}$, dry ice/acetone.

TLC was performed on $0.25 \mathrm{~mm}$ E. Merck silica gel 60 F254 plates and visualized under UV light $(254 \mathrm{~nm})$ or by staining with potassium permanganate $\left(\mathrm{KMnO}_{4}\right)$. Silica flash chromatography was performed on E. Merck 230-400 mesh silica gel 60. Preparative scale HPLC purification was carried out on a Waters 2545 HPLC using a Sunfire column $(150 \times 10 \mathrm{~mm})$ with a flowrate of $5 \mathrm{~mL} / \mathrm{min}$ and a 996 diode array detector or on a Varian PrepStar HPLC using a Varian Dynamax column $(250 \times 21.4 \mathrm{~mm})$ with a flow rate of $16.0 \mathrm{~mL} / \mathrm{min}$ and $\mathrm{UV}$ detection at $259 \mathrm{~nm}$. Lyophilization was performed using a Labconco Freezone 2.5 instrument.

IR spectra were recorded on a Perkin-Elmer model 1600 or Bruker Optics Tensor 27 FTIR spectrometer with peaks reported in $\mathrm{cm}^{-1}$. NMR spectra were recorded on Bruker Avance II 500, Avance II 600, DRX500, or AMX400 instruments at $24^{\circ} \mathrm{C}$. Chemical shifts are expressed in ppm relative to TMS $\left({ }^{1} \mathrm{H}, 0 \mathrm{ppm}\right)$ or solvent signals: $\mathrm{CDCl}_{3}\left({ }^{13} \mathrm{C}, 77.0 \mathrm{ppm}\right), \mathrm{C}_{6} \mathrm{D}_{6}\left({ }^{1} \mathrm{H}, 7.16 \mathrm{ppm}\right.$; $\left.{ }^{13} \mathrm{C}, 128.0 \mathrm{ppm}\right), \mathrm{CD}_{3} \mathrm{OD}\left({ }^{1} \mathrm{H}, 3.31 \mathrm{ppm} ;{ }^{13} \mathrm{C}, 49.0 \mathrm{ppm}\right)$ or acetone- $d_{6}\left({ }^{13} \mathrm{C}, 206.2 \mathrm{ppm}\right)$; coupling constants are expressed in Hz. Mass spectra were obtained at the MSKCC Analytical Core Facility on a PE SCIEX API 100 or Waters Acquity SQ mass spectrometer by electrospray (ESI) ionization or atmospheric pressure chemical ionization (AP-CI).

In vitro translation reactions were carried out using a $\mathrm{T} 7$ coupled rabbit reticulocyte system (Promega Cat. No. L4610). Luciferase T7 DNA was purchased from Promega (cat. no. L482A). Luminescence was detected using an LmaxII luminometer (Molecular Devices) using $50 \mu \mathrm{L}$ Luciferase reagent (Promega, Cat. No. E1500) with a $5 \mathrm{sec}$ delay and $10 \mathrm{sec}$ integration time.

Protein structural visualization, analysis, and graphic rendering was carried out using MacPyMOL v0.99 (http://pymol.sourceforge.net/) with a paid maintenance subscription.

Atom numbers in chemical structures and compound names herein correspond to standard nucleoside numbering system. Compounds not cited in the manuscript are numbered herein as S1-S27.

\footnotetext{
${ }^{9}$ Pangborn, A. B.; Giardello, M. A.; Grubbs, R. H.; Rosen, R. K.; Timmers, F. J. Organometallics 1996, 15, 1518 1520.
} 


\section{SYNTHESIS OF LINEAR AMINOACYL-AMS COMPOUNDS (1a-e)}

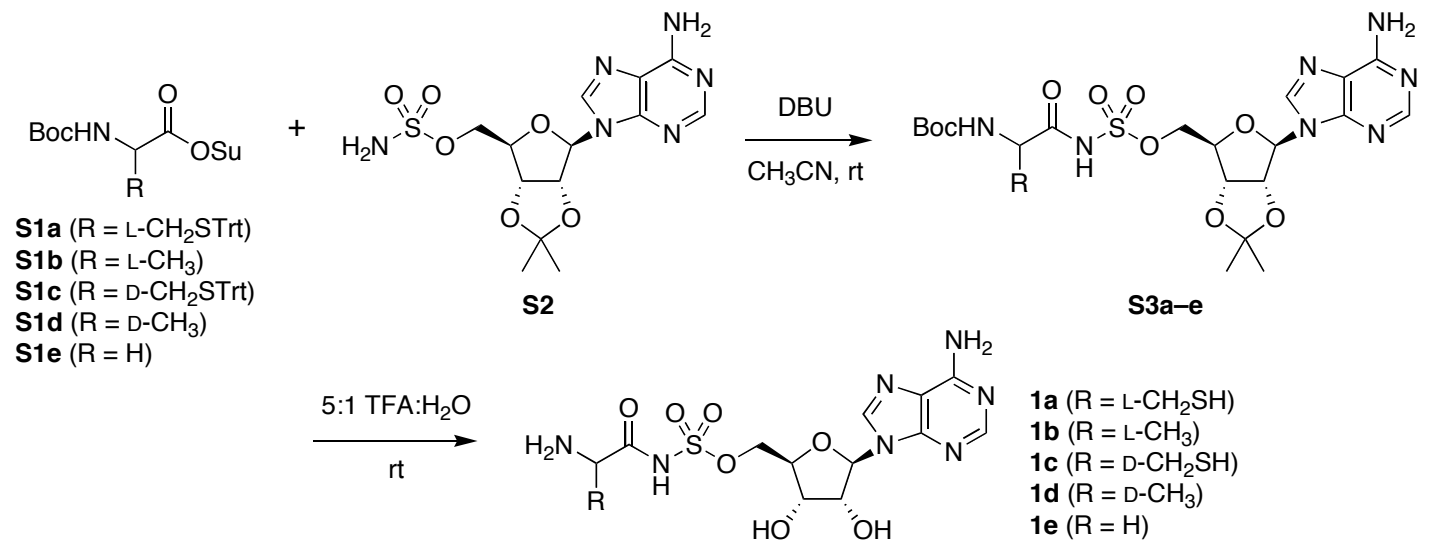

Figure S3. Synthesis of linear aminoacyl sulfamoyl adenosine analogs 1a-e. (Boc $=t$-butoxycarbonyl; DBU = 1,8-Diazabicyclo[5.4.0]undec-7-ene; Su = succinimidyl; TFA = 2,2,2-trifluoroacetic acid; Trt $=$ triphenylmethyl).

\section{GENERAL PROCEDURE FOR COUPLING $N$-HYDROXYSUCCINIMIDE ESTERS S1a-e WITH $5^{\prime}$-O $O$-SULFAMOYLADENOSINE $\mathrm{S} 2$.}

In a $20 \mathrm{~mL}$ vial, 2',3'-O-Isopropylidine-5'-O-sulfamoyl adenosine ${ }^{10} \mathbf{S 2}(20 \mathrm{mg}, 52 \mu \mathrm{mol}$, 1.0 equiv) and the $N$-hydroxysuccinimidyl ester ${ }^{11}$ (100 $\mu$ mol, 1.9 equiv) were disolved in $\mathrm{CH}_{3} \mathrm{CN}$ $(500 \mu \mathrm{L})$. DBU $(15.5 \mu \mathrm{L}, 100 \mu \mathrm{mol}, 1.9$ equiv) was added and the yellow solution was stirred at $\mathrm{rt}$ for $1 \mathrm{~h}$. The reaction was diluted in $9 \mathrm{~mL}$ EtOAc, washed with satd aq $\mathrm{NH}_{4} \mathrm{Cl}(3 \times 3 \mathrm{~mL})$ and satd aq $\mathrm{NaHCO}_{3}(3 \times 3 \mathrm{~mL})$, dried $\left(\mathrm{MgSO}_{4}\right)$, filtered, and concentrated by rotary evaporation. Purification with silica flash chromatography $\left(20: 1 \rightarrow 10: 1 \quad \mathrm{CHCl}_{3} / \mathrm{MeOH}\right)$ yielded the $\mathrm{N}$-aminoacyl sulfamoyl adenosines S3a-e. Analytical data for S3b, S3d, and S3e were in agreement with those reported previously. ${ }^{10}$

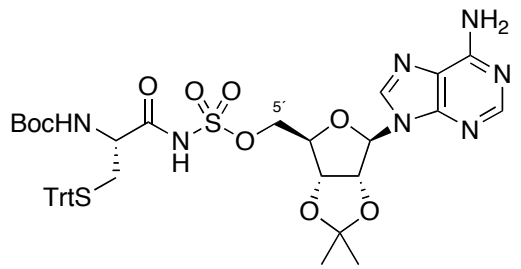

$\mathbf{2}^{\prime}, 3^{\prime}$ - $\boldsymbol{O}$-Isopropylidene-5' $\boldsymbol{O}-[\boldsymbol{N}$-( $\boldsymbol{N}$-Boc- $\boldsymbol{S}$-trityl-L-cysteyl)sulfamoyl $]$ adenosine (S3a). White solid (26 mg, 60\%). TLC: $R_{f} 0.08$ (9:1 $\left.\mathrm{CH}_{2} \mathrm{Cl}_{2} / \mathrm{MeOH}\right)$. IR (ZnSe, film): 3417 (N-H st), 3194 (N-H st), 2934, 1686 (C=O st), 1620, 1488, 1368 ( $\mathrm{SO}_{2}$ asym st), 1290, 1150 ( $\mathrm{SO}_{2}$ sym st), 1080, 854. ${ }^{1}$ H-NMR $\left(500 \mathrm{MHz}, \mathrm{CD}_{3} \mathrm{OD}\right): \delta 8.39(\mathrm{~s}, 1 \mathrm{H}), 8.19(\mathrm{~s}, 1 \mathrm{H}), 7.37-7.12(\mathrm{~m}, 15 \mathrm{H}), 6.20(\mathrm{~d}$, $1 \mathrm{H}, J=2.9), 5.27(\mathrm{dd}, 1 \mathrm{H}, J=6.0,3.2), 5.04(\mathrm{~d}, 1 \mathrm{H}, J=5.6), 4.49(\mathrm{~s}, 1 \mathrm{H}), 4.23(\mathrm{~s}, 2 \mathrm{H}), 4.12$ (s, $1 \mathrm{H}), 2.54(\mathrm{~d}, 2 \mathrm{H}, J=5.8), 1.58(\mathrm{~s}, 3 \mathrm{H}), 1.37(\mathrm{~m}, 12 \mathrm{H}) ; 4$ exchangeable $\mathrm{H}$ not observed. ${ }^{13}$ C-NMR (125 MHz, $\left.\mathrm{CD}_{3} \mathrm{OD}\right)$ : $\delta$ 179.0, 157.3, 154.0, 150.5, 146.3, 146.1, 141.5, 130.8, 128.9,

\footnotetext{
${ }^{10}$ Castro-Pichel, J.; Garcia-Lopez, M. T.; De las Heras, F. G. Tetrahedron 1987, 43, 383-389.

${ }^{11}$ Anderson, G. W.; Zimmerman, J. E.; Callahan, F. M. J. Am. Chem. Soc. 1964, 86, 1839-1842.
} 
127.7, 120.2, 115.3, 91.9, 85.8, 85.4, 83.3, 80.5, 70.0, 67.5, 37.6, 36.7, 28.8, 27.5, 25.6. ESI-MS $\mathrm{m} / z$ (rel int): $\operatorname{pos} 832.1\left([\mathrm{M}+\mathrm{H}]^{+}, 100\right), 854.1\left([\mathrm{M}+\mathrm{Na}]^{+}, 79\right) ;$ neg $830.0\left([\mathrm{M}-\mathrm{H}]^{-}\right)$.

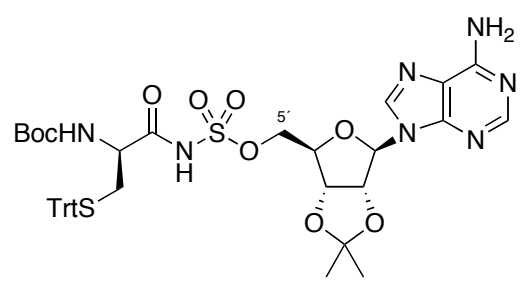

$\mathbf{2}^{\prime}, 3^{\prime}-O$-Isopropylidene-5' $O$-[ $N$-( $N$-Boc- $S$-trityl-D-cysteyl)sulfamoyl $]$ adenosine $(\mathrm{S3c})$. White solid (33 mg, 77\%). TLC: $R_{f} 0.08\left(9: 1 \mathrm{CH}_{2} \mathrm{Cl}_{2} / \mathrm{MeOH}\right)$. IR (ZnSe, film): $3417(\mathrm{~N}-\mathrm{H}$ st), 3190 ( $\mathrm{N}-\mathrm{H}$ st), 2865, 1680 (C=O st), 1618, 1487, 1366 ( $\mathrm{SO}_{2}$ asym st), 1293, $1149\left(\mathrm{SO}_{2} \mathrm{sym} \mathrm{st}\right), 1076$, 852. ${ }^{1}$ H-NMR $\left(500 \mathrm{MHz}, \mathrm{CD}_{3} \mathrm{OD}\right): \delta 8.38(\mathrm{~s}, 1 \mathrm{H}), 8.19(\mathrm{~s}, 1 \mathrm{H}), 7.36-7.11(\mathrm{~m}, 15 \mathrm{H}), 6.19(\mathrm{~d}$, $1 \mathrm{H}, J=3.8), 5.25(\mathrm{~m}, 1 \mathrm{H}), 5.04(\mathrm{dd}, 1 \mathrm{H}, J=5.9,2.2), 4.48(\mathrm{~s}, 1 \mathrm{H}), 4.22(\mathrm{~s}, 2 \mathrm{H}), 4.10(\mathrm{~m}, 1 \mathrm{H})$, $2.55(\mathrm{~m}, 2 \mathrm{H}), 1.57(\mathrm{~s}, 3 \mathrm{H}), 1.43(\mathrm{~s}, 9 \mathrm{H}), 1.29(\mathrm{~s}, 3 \mathrm{H}) ; 4$ exchangeable $\mathrm{H}$ not observed. ${ }^{13} \mathrm{C}$-NMR $\left(125 \mathrm{MHz}, \mathrm{CD}_{3} \mathrm{OD}\right): \delta 179.1,157.3,154.0,150.5,146.3,146.1,141.5,130.8,128.9,127.7$, 120.2, 115.4, 91.9, 85.8, 85.7, 83.3, 80.6, 70.0, 67.4, 36.7, 35.7, 28.9, 27.6, 25.7. ESI-MS $\mathrm{m} / \mathrm{z}$ (rel int): pos $832.1\left([\mathrm{M}+\mathrm{H}]^{+}, 100\right), 853.8\left([\mathrm{M}+\mathrm{Na}]^{+}, 73\right)$; neg $830.0\left([\mathrm{M}-\mathrm{H}]^{-}\right)$.

\section{General Procedure for Global Deprotection of S3a-e.}

In a $20 \mathrm{~mL}$ glass vial, the protected aminoacyl-AMS compound S3a-e (50 $\mu \mathrm{mol})$ was dissolved in cool $\left(10{ }^{\circ} \mathrm{C}\right)$ 5:1 TFA/water $\left(1 \mathrm{~mL}\right.$; containing $5 \% \mathrm{Et}_{3} \mathrm{SiH}$ for the $\mathbf{S 3 a}$ and $\mathbf{S 3 b}$ reactions $)$ and the reaction was stirred at $\mathrm{rt}$ for $30 \mathrm{~min}$. The solution was cooled to $0{ }^{\circ} \mathrm{C}$, then $\mathrm{MeOH}(5 \mathrm{~mL})$ was added and the mixture was concentrated by rotary evaporation. The residue was evaporated from $\mathrm{MeOH}(5 \mathrm{~mL})$ two more times. The crude product was dissolved in water and purified via preparative HPLC $\left(\mathrm{C}_{18} ; 10 \times 150 \mathrm{~mm}\right.$; gradient $0-20 \% \mathrm{CH}_{3} \mathrm{CN}$ in $\mathrm{H}_{2} \mathrm{O}$ with $0.05 \%$ TFA over 20 $\mathrm{min} ; 5 \mathrm{~mL} / \mathrm{min}$ ). Analytical data for $\mathbf{1 b}, \mathbf{1 d}$, and $\mathbf{1 e}$ were in agreement with those reported previously. ${ }^{10}$

L-Cys-AMS (1a) and D-Cys-AMS (1c) were found to dimerize rapidly upon dissolution in DMSO via disulfide formation (LC-MS), ${ }^{12}$ but were likewise reduced rapidly ( $\leq 2 \mathrm{~min}$ ) in the presence of the $1 \mathrm{mM}$ TCEP (tris[2-carboxyethyl]phosphine) used in the assay buffer (see SECTION H below). Linear aminoacyl-AMS compounds 1a-d were also found to decompose slowly upon extended storage over several weeks in DMSO at $-20{ }^{\circ} \mathrm{C}$ via precedented $N 3 \rightarrow C 5^{\prime}$-cyclonucleoside formation. ${ }^{13}$ However, control experiments confirmed that the isolated cyclonucleoside byproduct does not inhibit HMWP2. This decomposition pathway was not observed for macrocycles $\mathbf{2 a}-\mathbf{e}$.

\footnotetext{
${ }^{12}$ Tam, J. P.; Wu, C. R.; Liu, W.; Zhang, J. W. J. Am. Chem. Soc. 1991, 113, 6657-6662.

${ }^{13}$ Shuman, D. A.; Robins, M. J.; Robins, R. K. J. Am. Chem. Soc. 1970, 92, 3434-3440.
} 


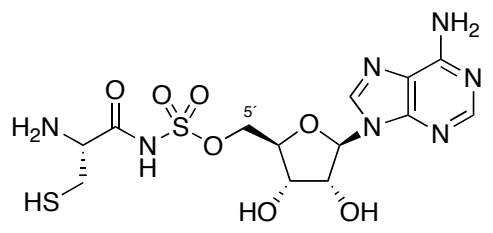

5'-O-[N-(L-Cysteyl)sulfamoyl]adenosine (1a, L-Cys-AMS). White solid (5.3 mg, 42\%). HPLC: $t_{\text {ret }}=8.2 \mathrm{~min}$. IR (ZnSe, film): $3436(\mathrm{~N}-\mathrm{H}$ st, O-H st), $3126(\mathrm{~N}-\mathrm{H}$ st), 2947, $1675(\mathrm{C}=\mathrm{O}$ st), 1506, 1420, 1385 ( $\mathrm{SO}_{2}$ asym st), 1292, 1199, 1134 ( $\mathrm{SO}_{2}$ sym st), 1020, 837. ${ }^{1}$ H-NMR $\left(500 \mathrm{MHz}, \mathrm{CD}_{3} \mathrm{OD}+\operatorname{trace} \mathrm{D}_{2} \mathrm{O}\right): \delta 8.64(\mathrm{~d}, 1 \mathrm{H}, J=3.9), 8.56(\mathrm{~s}, 1 \mathrm{H}), 6.10(\mathrm{~d}, 1 \mathrm{H}, J=3.2), 4.63$ $(\mathrm{t}, 1 \mathrm{H}, J=5.1), 4.49-4.30(\mathrm{~m}, 4 \mathrm{H}), 4.10(\mathrm{dd}, 1 \mathrm{H}, J=5.9,4.31), 3.31-3.18(\mathrm{~m}, 2 \mathrm{H}) ; 8$ exchangeable $\mathrm{H}$ not observed. ${ }^{13} \mathbf{C}-\mathbf{N M R}\left(125 \mathrm{MHz}, \mathrm{CD}_{3} \mathrm{OD}\right): \delta 173.6,153.2,150.6,147.5$, 143.9, 120.5, 90.3, 85.0, 76.8, 72.4, 69.8, 58.5, 26.9. ESI-MS $\mathrm{m} / \mathrm{z}$ (rel int): pos 449.8 $\left([\mathrm{M}+\mathrm{H}]^{+}, 100\right)$; neg $447.7\left([\mathrm{M}-\mathrm{H}]^{-}\right)$.

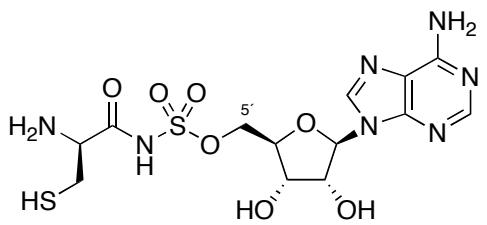

5'-O-[N-(D-Cysteyl)sulfamoyl]adenosine (1c, D-Cys-AMS). White solid $(9.6 \mathrm{mg}, 60 \%)$. HPLC: $t_{\mathrm{ret}}=8.3 \mathrm{~min}$. IR (ZnSe, film): $3436(\mathrm{~N}-\mathrm{H}$ st, O-H st), $3128(\mathrm{~N}-\mathrm{H}$ st), 2940, $1678(\mathrm{C}=\mathrm{O}$ st), 1508, 1422, 1382 ( $\mathrm{SO}_{2}$ asym st), 1295, 1202, 1139 ( $\mathrm{SO}_{2}$ sym st), 1020, 840. ${ }^{1} \mathbf{H}-\mathbf{N M R}$ $\left(500 \mathrm{MHz}, \mathrm{CD}_{3} \mathrm{OD}\right): \delta 8.68(\mathrm{~s}, 1 \mathrm{H}), 8.40(\mathrm{~s}, 1 \mathrm{H}), 6.10(\mathrm{~d}, 1 \mathrm{H}, J=3.2), 4.66(\mathrm{t}, 1 \mathrm{H}, J=4.0)$, 4.45-4.33 (m, 4H), $3.94(\mathrm{dd}, 1 \mathrm{H}, 4.8,3.4), 3.13-3.03(\mathrm{~m}, 2 \mathrm{H}) ; 8$ exchangeable $\mathrm{H}$ not observed. ${ }^{13}$ C-NMR $\left(125 \mathrm{MHz}, \mathrm{CD}_{3} \mathrm{OD}\right): \delta 173.5,153.1,150.6,147.2,143.9,120.5,90.4,85.0,76.8,72.4$, 69.9, 58.5, 26.9. ESI-MS $\mathrm{m} / z$ (rel int): pos 449.9 ([M+H] $\left.]^{+}, 100\right), 471.9$ ([M+Na $\left.]^{+}, 5\right)$; neg 447.8 $\left([\mathrm{M}-\mathrm{H}]^{-}\right)$. 


\section{E. SYNTHESIS OF CYCLO ${ }^{8 C_{2} \beta}$-ALA-AMS MACROCYCLES $(2 \mathrm{a}, 2 \mathrm{c})$}

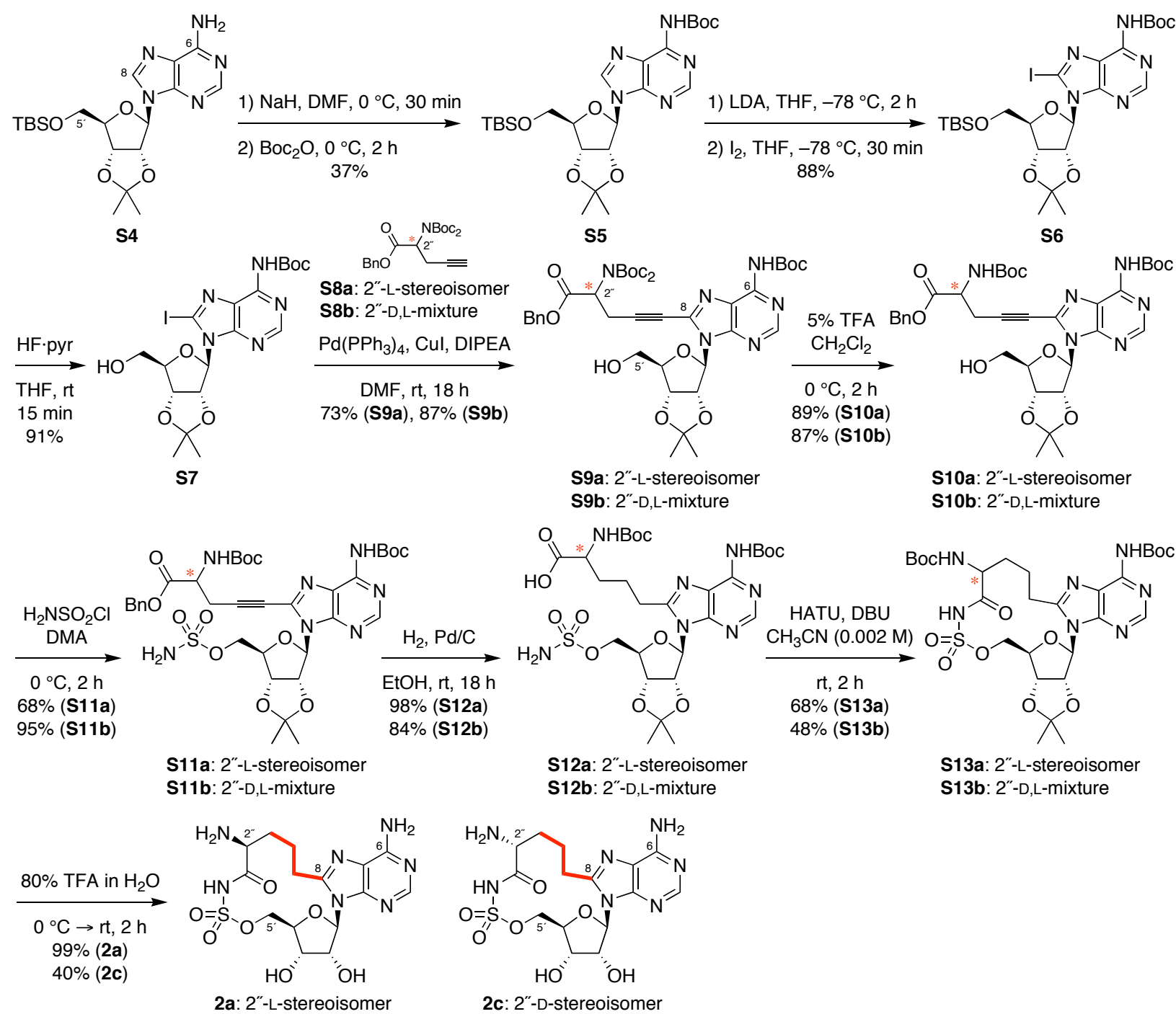

Figure S4. Synthesis of macrocycles $2 a$ and $2 c$ having a 2-carbon linker (red). Macrocycles $2 a$ and 2c were synthesized from the known adenosine acetonide S4. ${ }^{14}$ Bis(Boc)-protected propargyl glycines S8 were used for the Sonogashira couplings (to avoid cyclization of the nitrogen functionality) to form S9. Since the 2"-bis(Boc)-protected congener of macrocycle $\mathbf{S 1 3}$ proved highly sensitive to hydrolysis, one of the 2"-Boc protecting groups was removed selectively to form 2"-mono(Boc)-protected intermediate S10, which was then carried through the remainder of the synthesis as shown. The D-stereoisomer $\mathbf{2 c}$ was obtained by synthesis using racemic propargyl glycine S8b, followed by HPLC separation of the diasteromers at the conclusion of the synthesis. (DIPEA $=N, N$-diisopropylethylamine; DMA $=N, N$-dimethylacetamide; DMF $=N, N$-dimethylformamide; HATU $=0$-(7-Azabenzotriazole-1-yl)- $N, N^{\prime}, N^{\prime}, N^{\prime}$-tetramethyluronium hexafluorophosphate; $\mathrm{LDA}=$ lithium diisopropylamide; TBS = tert-butyldimethylsilyl; THF = tetrahydrofuran).

\footnotetext{
${ }^{14}$ Somu, R. V.; Boshoff, H.; Qiao, C.; Bennett, E. M.; Barry, C. E., III; Aldrich, C. C. J. Med. Chem. 2006, 49, $31-$
} 34. 


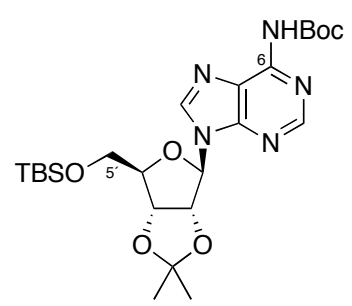

6- $\mathrm{N}$ - $t$-Butoxycarbonyl-5' $-O$ - $t$-butyldimethylsilyl-2, $\mathbf{2}^{\prime}-O$-isopropylideneadenosine (S5). In a $500 \mathrm{~mL}$ roundbottom flask, adenosine derivative $\mathbf{S 4}(7.8 \mathrm{~g}, 18.5 \mathrm{mmol})$ was dissolved in anhyd DMF (150 mL) and cooled to $0{ }^{\circ} \mathrm{C}$. Solid NaH (1.06 g, $44.1 \mathrm{mmol}, 2.4$ equiv) was added in two portions. After stirring at $0{ }^{\circ} \mathrm{C}$ for $30 \mathrm{~min}, \mathrm{Boc}_{2} \mathrm{O}(1.36 \mathrm{~g}, 25.2 \mathrm{mmol}, 1.4$ equiv) was added in three portions. After $2 \mathrm{~h}$, the reaction was quenched with $1 \mathrm{~N}$ aq $\mathrm{HCl}(70 \mathrm{~mL})$ and extracted with EtOAc $(2 \times 150 \mathrm{~mL})$. The combined organic extracts were washed with $1 \mathrm{~N}$ aq $\mathrm{NaOH}$ $(2 \times 50 \mathrm{~mL})$ and satd aq $\mathrm{NH}_{4} \mathrm{Cl}(2 \times 50 \mathrm{~mL})$, dried $\left(\mathrm{MgSO}_{4}\right)$, filtered, and concentrated by rotary evaporation. Purification by silica flash chromatography $(2: 1 \rightarrow 1: 1$ hexanes/EtOAc) yielded S5 (3.56 g, 37\%) as a foamy oil. Analytical data for S5 were in agreement with those reported previously. ${ }^{14}$

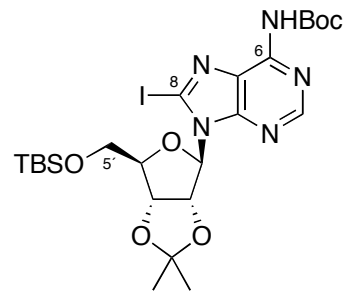

\section{6- $\mathrm{N}$-t-Butoxycarbonyl-5' $-O$-t-butyldimethylsilyl-8-iodo- $2^{\prime}, 3^{\prime}-O$-isopropylideneadenosine}

(S6). In a $250 \mathrm{~mL}$ roundbottom flask, $i-\mathrm{Pr}_{2} \mathrm{NH}(2.9 \mathrm{~mL}, 20.4 \mathrm{mmol}, 3.0$ equiv) was dissolved in anhyd THF $(13 \mathrm{~mL})$. The solution was cooled to $-78^{\circ} \mathrm{C}$ and $n$-BuLi $(1.5 \mathrm{M}$ in hexanes, $13.6 \mathrm{~mL}, 3.0$ equiv) was added. After $30 \mathrm{~min}$, a solution of adenosine derivative $\mathbf{S 5}$ (3.56 g, $6.82 \mathrm{mmol})$ in anhyd THF $(13 \mathrm{~mL})$ was added via cannula and the resulting orange solution was stirred at $-78^{\circ} \mathrm{C}$ for $2 \mathrm{~h}$. A solution of $\mathrm{I}_{2}(3.44 \mathrm{~g}, 13.6 \mathrm{mmol}, 2.0$ equiv) in anhyd THF $(27 \mathrm{~mL})$ was added via cannula and the resulting purple solution was stirred at $-78{ }^{\circ} \mathrm{C}$ for $30 \mathrm{~min}$. The reaction was quenched by addition of satd aq $\mathrm{NH}_{4} \mathrm{Cl}(4 \mathrm{~mL})$ followed by satd aq $\mathrm{Na}_{2} \mathrm{~S}_{2} \mathrm{O}_{4}$ $(30 \mathrm{~mL})$ and warmed to $\mathrm{rt}$. The mixture was extracted with EtOAc $(3 \times 100 \mathrm{~mL})$. The combined organic extracts were washed with satd aq $\mathrm{NH}_{4} \mathrm{Cl}(2 \times 50 \mathrm{~mL}), 1 \mathrm{~N} \mathrm{NaOH}(2 \times 50 \mathrm{~mL})$, and brine $(50 \mathrm{~mL})$, dried $\left(\mathrm{MgSO}_{4}\right)$, filtered, and concentrated by rotary evaporation. Purification by silica flash chromatography (3:1 hexanes/EtOAc) yielded 8-iodoadenosine derivative S6 (3.83 g, $88 \%$ ) as a light yellow solid.

TLC: $R_{f} 0.62$ (1:1 hexanes/EtOAc). IR (NaCl, film): 3451 (N-H st), 2977, 1762 (C=O st), 1612, 1430, 1370, 1326, 1278, 1230, 1157, 1066, 875, 793. ${ }^{1} \mathbf{H}-\mathbf{N M R}\left(400 \mathrm{MHz}, \mathrm{CDCl}_{3}\right): \delta 8.64(\mathrm{~s}$, $1 \mathrm{H}), 7.93(\mathrm{~s}, 1 \mathrm{H}), 6.09(\mathrm{~d}, 1 \mathrm{H}, J=2.0), 5.81(\mathrm{dd}, 1 \mathrm{H}, J=6.3,2.0), 5.15$, (dd, 1H, $J=6.3,3.2)$, 4.29 (m, 1H), 3.72 (dd, 1H, $J=10.6,6.6), 3.61$ (dd, 1H, $J=10.6,6.4), 1.62$ (s, 3H), 1.54 (s, 9H), $1.40(\mathrm{~s}, 3 \mathrm{H}), 0.82(\mathrm{~s}, 9 \mathrm{H}),-0.08(\mathrm{~s}, 6 \mathrm{H}) .{ }^{13} \mathbf{C}-\mathbf{N M R}\left(100 \mathrm{MHz}, \mathrm{CDCl}_{3}\right): \delta 152.7,150.8,149.6$, 148.5, 124.5, 114.1, 103.7, 93.7, 88.2, 82.7, 82.5, 82.1, 63.0, 28.0, 27.2, 25.8, 25.4, 18.3, -5.4, -5.5. ESI-MS $m / z$ (rel int): pos $647.9\left([\mathrm{M}+\mathrm{H}]^{+}, 100\right), 669.8\left([\mathrm{M}+\mathrm{Na}]^{+}, 13\right)$; neg $645.9\left([\mathrm{M}-\mathrm{H}]^{-}\right)$. 


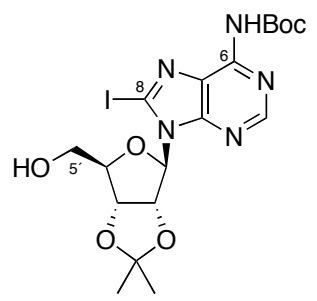

6- $\boldsymbol{N}$-t-Butoxycarbonyl-8-iodo-2', $\mathbf{3}^{\prime}$ - $\boldsymbol{O}$-isopropylideneadenosine (S7). In a $50 \mathrm{~mL}$ plastic Falcon tube, 8-iodoadenosine derivative $\mathbf{S 6}(1.93 \mathrm{~g}, 2.98 \mathrm{mmol})$ was dissolved in anhyd THF (25 mL) and HF.pyr (2.7 mL, $150 \mathrm{mmol}, 50$ equiv) was added dropwise at rt. After $15 \mathrm{~min}$, the reaction was quenched by addition to cold $1 \mathrm{~N} \mathrm{NaOH}(30 \mathrm{~mL})$. The mixture was extracted with $\mathrm{CH}_{2} \mathrm{Cl}_{2}(2 \times 100 \mathrm{~mL})$ and the combined organic extracts were washed with satd $\mathrm{NH}_{4} \mathrm{Cl}$ $(2 \times 30 \mathrm{~mL})$, dried $\left(\mathrm{MgSO}_{4}\right)$, filtered, and concentrated by rotary evaporation. Purification by silica flash chromatography $\left(99: 1 \rightarrow 98: 2 \mathrm{CH}_{2} \mathrm{Cl}_{2} / \mathrm{MeOH}\right)$ yielded alcohol $\mathbf{S 7}(1.44 \mathrm{~g}, 91 \%)$ as a white solid.

TLC: $R_{f} 0.13$ (1:1 hexanes/EtOAc). IR (NaCl, film): $3287(\mathrm{~N}-\mathrm{H}$ st, O-H st), 2977, 1762 (C=O st), 1612, 1430, 1370, 1326, 1278, 1230, 1157, 1066, 875, 793. ${ }^{1} \mathbf{H}-\mathbf{N M R}\left(500 \mathrm{MHz}, \mathrm{CDCl}_{3}\right)$ : $\delta 8.61(\mathrm{~s}, 1 \mathrm{H}), 7.99(\mathrm{~s}, 1 \mathrm{H}), 6.01(\mathrm{~d}, 1 \mathrm{H}, J=5.3), 5.82(\mathrm{~d}, 1 \mathrm{H}, J=10.9), 5.27(\mathrm{t}, 1 \mathrm{H}, J=5.5)$, $5.05(\mathrm{dd}, 1 \mathrm{H}, J=5.4,0.8), 4.52(\mathrm{~d}, 1 \mathrm{H}, J=0.9), 3.93(\mathrm{~d}, 1 \mathrm{H}, J=12.8), 3.76(\mathrm{~m}, 1 \mathrm{H}), 1.68(\mathrm{~s}$, 3H), 1.53 (s, 9H), 1.36 (s, 3H). ${ }^{13} \mathbf{C}-\mathbf{N M R}\left(125 \mathrm{MHz}, \mathrm{CDCl}_{3}\right): \delta 152.3,150.4,149.1,149.0$, 125.1, 114.1, 102.5, 95.7, 85.6, 82.7, 82.3, 81.5, 63.2, 28.0, 27.7, 25.4. ESI-MS $m / z$ (rel int): pos $533.8\left([\mathrm{M}+\mathrm{H}]^{+}, 28\right), 555.9\left([\mathrm{M}+\mathrm{Na}]^{+}, 100\right)$; neg $531.8\left([\mathrm{M}-\mathrm{H}]^{-}\right)$.

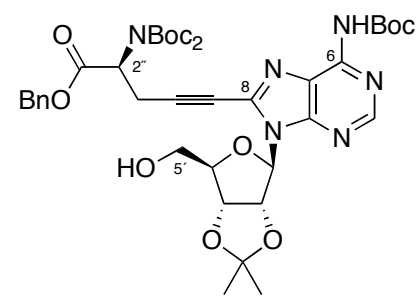

6- $N$-t-Butoxycarbonyl-8-(O-benzyl- $N, N$-bis[t-butoxycarbonyl]-L-2"-propargylglycin-5"-yl)$\mathbf{2}^{\prime}, \mathbf{3}^{\prime}$ - $\boldsymbol{O}$-isopropylideneadenosine (S9a). In a $25 \mathrm{~mL}$ roundbottom flask, 8-iodoadenosine derivative $\mathbf{S 7}(266 \mathrm{mg}, \quad 0.50 \mathrm{mmol})$ and $O$-Bn- $N, N$-bis(Boc)-L-2-propargylglycine ${ }^{15}$ S8a (242 mg, $0.6 \mathrm{mmol}, 1.2$ equiv) were dissolved in Ar-degassed anhyd DMF (5 mL). DIPEA (261 $\mu \mathrm{L}, 1.5 \mathrm{mmol}, 3.0$ equiv), CuI (38.0 mg, $0.2 \mathrm{mmol}, 0.4$ equiv), and $\mathrm{Pd}\left(\mathrm{PPh}_{3}\right)_{4}(115 \mathrm{mg}$, $0.1 \mathrm{mmol}, 0.2$ equiv) ${ }^{16}$ were added and the reaction was stirred at $\mathrm{rt}$ for $18 \mathrm{~h}$. Satd aq $\mathrm{NH}_{4} \mathrm{Cl}$ $(20 \mathrm{~mL})$ was added and the mixture was extracted with EtOAc $(3 \times 70 \mathrm{~mL})$. The combined organic extracts were dried $\left(\mathrm{MgSO}_{4}\right)$, filtered and concentrated by rotary evaporation.

\footnotetext{
${ }^{15}$ Wolf, L. B.; Tjen, K. C. M. F.; ten Brink, H. T.; Blaauw, R. H.; Hiemstra, H.; Schoemaker, H. E.; Rutjes, F. P. J. T. Adv. Syn. Catal. 2002, 344, 70-83.

${ }^{16}$ Coulson, D. R. Inorg. Synth. 1972, 13, 121-124.
} 
Purification by silica flash chromatography $(1: 1 \rightarrow 4: 5$ hexanes/EtOAc) yielded 8-alkynyladenosine derivative S9a (294 mg, 73\%).

TLC: $R_{f} 0.15$ (1:1 hexanes/EtOAc). IR (NaCl, film): 3286 (N-H st, O-H st), 2980, 2936, 2246 (C $\equiv \mathrm{C}$ st), 1754 (C=O st), 1704 (C=O st), 1607, 1480, 1455, 1428, 1368, 1228, 1143, 1111, 972, 851. ${ }^{1} \mathbf{H}-N M R\left(500 \mathrm{MHz}, \mathrm{CDCl}_{3}\right): \delta 8.71(\mathrm{~s}, 1 \mathrm{H}), 7.97(\mathrm{~s}, 1 \mathrm{H}), 7.35-7.31(\mathrm{~m}, 5 \mathrm{H}), 6.22(\mathrm{~d}, 1 \mathrm{H}$, $J=5.2), 5.87(\mathrm{~d}, 1 \mathrm{H}, J=10.1), 5.32(\mathrm{dd}, 1 \mathrm{H}, J=5.5,3.5), 5.23-5.16(\mathrm{~m}, 3 \mathrm{H}), 5.07(\mathrm{~d}, 1 \mathrm{H}, J=$ $5.5), 4.50(\mathrm{~s}, 1 \mathrm{H}), 3.97(\mathrm{~d}, 1 \mathrm{H}, J=13.0), 3.78(\mathrm{~d}, 1 \mathrm{H}, J=11.5,1.5), 3.43(\mathrm{~m}, 1 \mathrm{H}), 3.24(\mathrm{~m}, 1 \mathrm{H})$, $1.68(\mathrm{~s}, 3 \mathrm{H}), 1.52(\mathrm{~s}, 9 \mathrm{H}), 1.42(\mathrm{~s}, 18 \mathrm{H}), 1.36(\mathrm{~s}, 3 \mathrm{H}) .{ }^{13} \mathbf{C}-\mathbf{N M R}\left(125 \mathrm{MHz}, \mathrm{CDCl}_{3}\right): \delta 168.8$, 153.2, 151.9, 150.1, 149.1, 148.9, 136.2, 135.1, 128.5, 128.3, 128.2, 122.0, 114.0, 95.8, 92.8, 85.7, 83.8, 82.8, 82.6, 81.5, 70.6, 67.4, 63.2, 56.4, 28.0, 27.9, 27.7, 25.4, 21.5. ESI-MS $\mathrm{m} / z$ (rel int): $\operatorname{pos} 809.1\left([\mathrm{M}+\mathrm{H}]^{+}, 100\right), 831.0\left([\mathrm{M}+\mathrm{Na}]^{+}, 90\right)$; neg $806.9\left([\mathrm{M}-\mathrm{H}]^{-}, 100\right), 843.4\left([\mathrm{M}+\mathrm{Cl}]^{-}\right.$, $15)$.

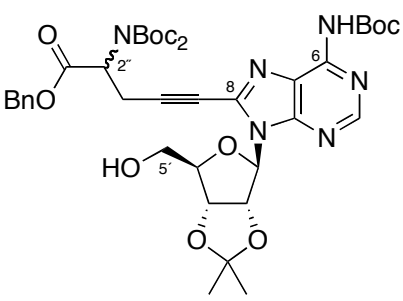

6- $N$-t-Butoxycarbonyl-8-( $O$-benzyl- $N, N$-bis[t-butoxycarbonyl]-DL-2"-propargylglycin-5"-yl)$\mathbf{2}^{\prime}, \mathbf{3}^{\prime}$ - $\boldsymbol{O}$-isopropylideneadenosine (S9b). Prepared from S7 as above using DL-propargylglycine S8b. White powder $(381 \mathrm{mg}, 87 \%)$. TLC: $R_{f} 0.15$ (1:1 hexanes/EtOAc). IR (NaCl, film): 3286 (N-H, O-H st), 2982, 2243 (C $\equiv \mathrm{C}$ st), 1751 (C=O st), 1694 (C=O st), 1608, 1479, 1369, 1231, 1265, 1143, 1111, 909, 851. ${ }^{1} \mathbf{H}-\mathbf{N M R}\left(500 \mathrm{MHz}, \mathrm{CDCl}_{3}\right): \delta 8.71(\mathrm{~s}, 1 \mathrm{H}), 7.99(\mathrm{~s}, 1 \mathrm{H}), 7.34(\mathrm{~m}$, $5 \mathrm{H}), 6.27$ (d, 0.5H, $J=4.2$, D-stereoisomer), 6.22 (d, 0.5H, $J=4.1$, L-stereoisomer), 5.96 (dd, $1 \mathrm{H}, J=13.5,11.6), 5.34(\mathrm{~m}, 1 \mathrm{H}), 5.21(\mathrm{~m}, 3 \mathrm{H}), 5.08(\mathrm{~m}, 1 \mathrm{H}), 4.52(\mathrm{~m}, 1 \mathrm{H}), 3.97(\mathrm{~d}, 1 \mathrm{H}, J=$ 12.8), 3.79 (m, 1H), $3.43(\mathrm{dd}, 1 \mathrm{H}, J=14.8,5.1), 3.24(\mathrm{~m}, 1 \mathrm{H}), 1.75(\mathrm{~s}, 3 \mathrm{H}), 1.67-1.40(\mathrm{~m}, 30 \mathrm{H})$. ${ }^{13}$ C-NMR $\left(125 \mathrm{MHz}, \mathrm{CDCl}_{3}\right): \delta 168.8,153.2,151.9 *, 151.8,150.1,149.1 .148 .9,136.2,135.1$, $128.5,128.4,128.2,122.0,114.1,114.0^{*}, 95.8,92.9,85.7,83.9,83.8^{*}, 83.0,82.8,82.7^{*}, 81.6$, $70.6,67.5,63.3,56.4,28.0,27.9,27.8,25.5^{*}, 25.3,21.6,21.4^{*} ; 6$ resolved peaks arising specifically from the L-diastereomer S9a above are indicated with a *. ESI-MS $m / z$ (rel int): pos $809.0\left([\mathrm{M}+\mathrm{H}]^{+}, 60\right), 831.04\left([\mathrm{M}+\mathrm{Na}]^{+}, 100\right)$; neg $807.1\left([\mathrm{M}-\mathrm{H}]^{-}, 100\right), 843.4\left([\mathrm{M}+\mathrm{Cl}]^{-}, 15\right)$.

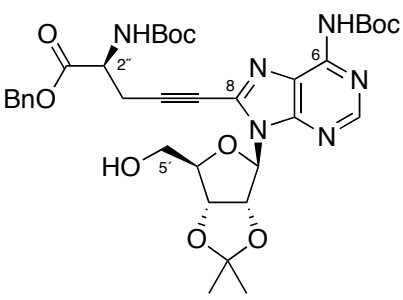

6- $N$ - $t$-Butoxycarbonyl-8-( $O$-benzyl- $N$ - $t$-butoxycarbonyl-L-2"-propargylglycin-5"-yl)-2',3'- $O$ isopropylideneadenosine (S10a). In a $15 \mathrm{~mL}$ roundbottom flask, 2 - $N, N$-bis-Boc-8-alkynyladenosine S9a $(273 \mathrm{mg}, 0.337 \mathrm{mmol})$ was dissolved in $\mathrm{CH}_{2} \mathrm{Cl}_{2}(3 \mathrm{~mL})$. The solution was cooled 
to $0{ }^{\circ} \mathrm{C}$ and TFA $(30 \mu \mathrm{L})$ was added. After stirring for $2 \mathrm{~h}, \mathrm{MeOH}(10 \mathrm{~mL})$ was added and the mixture was concentrated by rotary evaporation then azeotroped twice from toluene. Purification by silica flash chromatography (11:10 hexanes/EtOAc) yielded 2"- $N$-Boc-8-alkynyladenosine S10a (213 $\mathrm{mg}, 89 \%)$ as a white solid.

TLC: $R_{f} 0.13$ (1:1 hexanes/EtOAc). IR (NaCl, film): 3298 (N-H st, O-H st), 2980, 2245 (C $\equiv \mathrm{C}$ st), 1747 (C=O st), 1714 (C=O st), 1608, 1514, 1455, 1428, 1368, 1329, 1233, 1148, 1110, 851. ${ }^{1}$ H-NMR $\left(500 \mathrm{MHz}\right.$, acetone- $\left.d_{6}\right)$ : $\delta 8.61(\mathrm{br}, 1 \mathrm{H}), 7.45-7.20(\mathrm{~m}, 5 \mathrm{H}), 6.31(\mathrm{~d}, 1 \mathrm{H}, J=3.7), 5.48$ $(\mathrm{d}, 1 \mathrm{H}, J=5.7), 5.26(\mathrm{~s}, 2 \mathrm{H}), 5.12(\mathrm{dd}, 1 \mathrm{H}, J=6.0,2.4), 4.60(\mathrm{dd}, 1 \mathrm{H}, J=12.1,5.8), 4.36$ (dd, $1 \mathrm{H}, J=6.3,3.7), 3.75$ (dd, $1 \mathrm{H}, J=12.1,3.9), 3.66$ (dd, 1H, $J=12.1,4.0), 3.26-3.21(\mathrm{~m}, 2 \mathrm{H})$, $1.62(\mathrm{~s}, 3 \mathrm{H}), 1.55(\mathrm{~s}, 9 \mathrm{H}), 1.42(\mathrm{~s}, 9 \mathrm{H}), 1.38(\mathrm{~s}, 3 \mathrm{H}) ; 3$ exchangeable $\mathrm{H}$ not observed. ${ }^{13} \mathbf{C}-\mathbf{N M R}$ $\left(125 \mathrm{MHz}\right.$, acetone- $\left.d_{6}\right)$ : $\delta 171.2,156.2(2 \mathrm{C}), 152.9,151.4,151.3,151.0,136.8(2 \mathrm{C}), 129.3$, $128.9,123.6,114.6,95.7,92.4,87.9,83.8,82.6,81.7,80.0,72.3,67.7,63.2,31.0,28.5,28.3$, 27.9, 25.7, 23.5. ESI-MS $\mathrm{m} / z$ (rel int): pos $709.1\left([\mathrm{M}+\mathrm{H}]^{+}, 100\right), 732.0\left([\mathrm{M}+\mathrm{Na}]^{+}, 8\right)$; neg 743.1 $\left([\mathrm{M}+\mathrm{Cl}]^{-}\right)$.

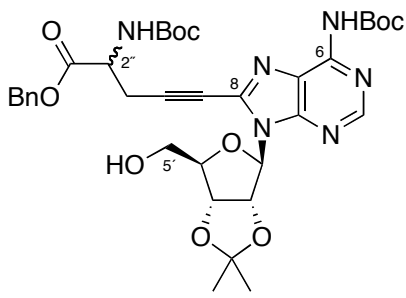

6- $N$ - $t$-Butoxycarbonyl-8-(O-benzyl- $N$-t-butoxycarbonyl-DL-2"-propargylglycin-5"'yl)-2',3'$\boldsymbol{O}$-isopropylideneadenosine $(\mathbf{S 1 0 b})$. Prepared from $\mathbf{S} 9 \mathbf{b}$ as above. White powder $(381 \mathrm{mg}$, 87\%). TLC: $R_{f} 0.13$ (1:1 hexanes/EtOAc). IR (NaCl, film): 3298 (N-H st, O-H st), 2980, 2936, 2243 (C $\equiv \mathrm{C}$ st), 1737 (C=O st), 1701 (C=O st), 1605, 1514, 1456, 1427, 1367, 1326, 1226, 1140, 1109, 851. ${ }^{1} \mathbf{H}-\mathbf{N M R}\left(500 \mathrm{MHz}\right.$, acetone- $\left.d_{6}\right)$ : $\delta 8.59(\mathrm{br}, 1 \mathrm{H}), 7.34(\mathrm{~m}, 5 \mathrm{H}), 6.30(\mathrm{~m}, 1 \mathrm{H}), 5.46$ $(\mathrm{m}, 1 \mathrm{H}), 5.28(\mathrm{~m}, 2 \mathrm{H}), 5.12(\mathrm{~m}, 1 \mathrm{H}), 4.62(\mathrm{~m}, 1 \mathrm{H}), 4.37(\mathrm{~m}, 1 \mathrm{H}), 3.76(\mathrm{~m}, 1 \mathrm{H}), 3.68(\mathrm{~m}, 1 \mathrm{H})$, $3.22(2 \mathrm{H}, \mathrm{m}), 1.62(\mathrm{~m}, 3 \mathrm{H}), 1.52(\mathrm{~m}, 9 \mathrm{H}), 1.42-1.37(12 \mathrm{H}) ; 3$ exchangeable $\mathrm{H}$ not observed. ${ }^{13}$ C-NMR (125 MHz, acetone- $d_{6}$ ): $\delta 169.8,156.2$ (2C), 152.9, 151.2, 151.1, 150.9, 136.8 (2C), $129.3,128.9^{*}, 128.8,123.5,114.6^{*}, 114.5,95.9,92.43,92.37 *, 87.7,84.1,82.6,81.7,80.0,71.9$, $67.8,63.2,32.3,28.5,28.3,28.0,25.7,23.3 ; 3$ resolved peaks arising specifically from the L-diastereomer S10a above are indicated with a *. ESI-MS $m / z$ (rel int): pos $709.2\left([\mathrm{M}+\mathrm{H}]^{+}\right.$; neg $743.1\left([\mathrm{M}-\mathrm{H}]^{-}\right)$.

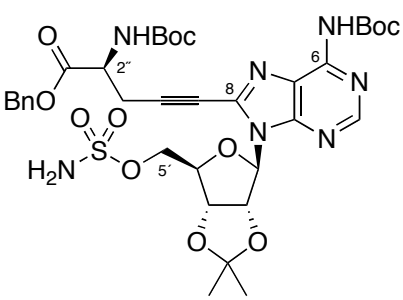

6- $N$ - $t$-Butoxycarbonyl-8-(O-benzyl- $N$-t-butoxycarbonyl-L-2"-propargylglycin-5"-yl)-2',3'-Oisopropylidene-5' $\mathbf{O}^{\prime}$-sulfamoyladenosine (S11a). In a $15 \mathrm{~mL}$ roundbottom flask, 8-alkynyl- 
adenosine derivative S10a $(198 \mathrm{mg}, 0.279 \mathrm{mmol})$ was dissolved in anhyd $N, N$-dimethylacetamide (DMA, $1 \mathrm{~mL}) .{ }^{17}$ The solution was cooled to $0{ }^{\circ} \mathrm{C}$ and sulfamoyl chloride ${ }^{18}(140 \mathrm{mg}$, $1.21 \mathrm{mmol}, 4.4$ equiv) was added. After stirring at $\mathrm{rt}$ for $2 \mathrm{hr}$, the reaction was quenched by addition of cold $1 \mathrm{~N} \mathrm{NaOH}(1 \mathrm{~mL})$ followed by satd aq $\mathrm{NH}_{4} \mathrm{Cl}(10 \mathrm{~mL})$ and the mixture was warmed to $\mathrm{rt}$ and extracted with EtOAc $(3 \times 70 \mathrm{~mL})$. The combined organic extracts were washed with satd aq $\mathrm{NH}_{4} \mathrm{Cl}(2 \times 5 \mathrm{~mL})$, dried $\left(\mathrm{MgSO}_{4}\right)$, filtered, and concentrated by rotary evaporation. Purification by silica flash chromatography (6:5 EtOAc/hexanes) yielded 8-alkynyl-5'-sulfamoyladenosine S11a with 6.7 equiv of inseparable DMA (150 mg, 68\%, corrected for 6.7 equiv DMA).

TLC: $R_{f} 0.13$ (1:1 hexanes/EtOAc). IR (NaCl, film): 3298 (N-H st), 2979, 2244 (C三C st), 1747 $(\mathrm{C}=\mathrm{O}$ st $), 1714(\mathrm{C}=\mathrm{O}$ st $), 1608,1368\left(\mathrm{SO}_{2}\right.$ asym st $), 1232,1183\left(\mathrm{SO}_{2}\right.$ sym st $), 1148,1062,1013$, 870. ${ }^{1} \mathrm{H}-\mathrm{NMR}\left(400 \mathrm{MHz}\right.$, acetone- $\left.d_{6}\right): \delta 9.26(\mathrm{~s}, 1 \mathrm{H}), 8.68(\mathrm{~s}, 1 \mathrm{H}), 7.51(\mathrm{~d}, 2 \mathrm{H}, J=7.2), 7.44$ $7.34(\mathrm{~m}, 3 \mathrm{H}), 6.85(\mathrm{~m}, 2 \mathrm{H}), 6.45(\mathrm{~d}, 1 \mathrm{H}, J=8), 5.76(\mathrm{dd}, 1 \mathrm{H}, J=6.3,1.6), 5.39(\mathrm{dd} 1 \mathrm{H}, J=6.2$, 3.6), 5.35 (s, 2H), 4.70 (dd, $1 \mathrm{H}, J=14.4,6.4), 4.55(\mathrm{~m}, 1 \mathrm{H}), 4.43(\mathrm{~m}, 1 \mathrm{H}), 4.28$ (dd, $1 \mathrm{H}, J=$ 10.5, 7.0), $3.30(\mathrm{~m}, 2 \mathrm{H}), 1.67$ (s, 3H), 1.61 (s, 9H), 1.49 (s, 9H), 1.46 (s 3H); 1 exchangeable $\mathrm{H}$ not observed. ${ }^{13} \mathbf{C}$-NMR $\left(100 \mathrm{MHz}\right.$, acetone- $\left.d_{6}\right)$ : $\delta 171.1,156.7,156.0,154.2,151.5,151.3$, 150.6, 137.3, 137.2, 129.7, 129.3, 123.6, 115.3, 96.2, 91.5, 86.6, 84.9, 83.3, 82.0, 81.4, 72.7, 69.7, 68.2, 53.8, 27.9, 27.7, 26.9, 24.9, 23.0. ESI-MS $\mathrm{m} / \mathrm{z}$ (rel int): pos $788.0\left([\mathrm{M}+\mathrm{H}]^{+}, 100\right)$, $810.0\left([\mathrm{M}+\mathrm{Na}]^{+}, 90\right)$; neg $786.1\left([\mathrm{M}-\mathrm{H}]^{-}, 100\right), 822.2\left([\mathrm{M}+\mathrm{Cl}]^{-}, 20\right)$.

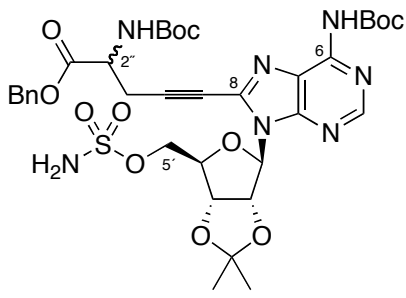

6- $N$-t-Butoxycarbonyl-8-(O-benzyl- $N$-t-butoxycarbonyl-DL-2"-propargylglycin-5"-yl)-2',3'$\boldsymbol{O}$-isopropylidene-5'-O-sulfamoyladenosine (S11b). Prepared from S10b as above. White powder (216 mg, 95\%, corrected for 4.8 equiv inseparable DMA). TLC: $R_{f} 0.13$ (1:1 hexanes/EtOAc). IR ( NaCl, film): 3303 (N-H st), 2980, 2937, 2245 (C $\equiv \mathrm{C}$ st), 1741 (C=O st), 1710 (C=O st), 1606, 1368 ( $\mathrm{SO}_{2}$ asym st), 1231, $1183\left(\mathrm{SO}_{2}\right.$ sym st), 1142, 1067, 997, 871. ${ }^{1}$ H-NMR $\left(500 \mathrm{MHz}\right.$, acetone- $\left.d_{6}\right): \delta 8.99(\mathrm{~s}, 1 \mathrm{H}), 8.66(\mathrm{~s}, 1 \mathrm{H}), 7.42(\mathrm{~m}, 5 \mathrm{H}), 6.78(\mathrm{~m}, 2 \mathrm{H}), 6.45$ $(\mathrm{m}, 1 \mathrm{H}), 5.74(\mathrm{~m}, 1 \mathrm{H}), 5.37(\mathrm{~m}, 3 \mathrm{H}), 4.70(\mathrm{~m}, 1 \mathrm{H}), 4.54(\mathrm{~m}, 1 \mathrm{H}), 4.43(\mathrm{~m}, 1 \mathrm{H}), 4.28(\mathrm{M}, 1 \mathrm{H})$, $3.36(\mathrm{~m}, 2 \mathrm{H}), 1.65(\mathrm{~m}, 12 \mathrm{H}), 1.45(\mathrm{~m}, 12 \mathrm{H}) ; 1$ exchangeable $\mathrm{H}$ not observed. ${ }^{13} \mathbf{C}$-NMR $\left(125 \mathrm{MHz}\right.$, acetone- $\left.d_{6}\right)$ : $\delta 170.1,156.7,156.2,153.8,151.3,151.1,150.9,150.6^{*}, 136.8,136.7$, 129.3, 128.9, 123.3, 115.0*, 114.9, 96.0, 91.0*, 90.9, 86.2*, 86.1, 84.6*, 84.5, 82.9*, 82.8, 82.0, $81.6,72.9,72.8^{*}, 69.4,67.7,53.4,27.9,27.5,26.8,24.80^{*}, 24.79,22.8 ; 8$ resolved peaks arising specifically from the L-diastereomer S11a above are indicated with a *. ESI-MS $m / z$ (rel int): pos $788.2\left([\mathrm{M}+\mathrm{H}]^{+}, 35\right), 810.0\left([\mathrm{M}+\mathrm{Na}]^{+}, 100\right)$; neg $786.0\left([\mathrm{M}-\mathrm{H}]^{-}\right)$.

\footnotetext{
${ }^{17}$ Okada, M.; Iwashita, S.; Koizumi, N. Tetrahedron Lett. 2000, 41, 7047-7051.

${ }^{18}$ Brodsky, B. H.; Du Bois, J. J. Am. Chem. Soc. 2005, 127, 15391-15393.
} 


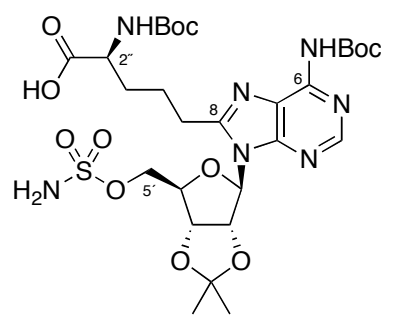

6- $N$-t-Butoxycarbonyl-8-( $N$-t-butoxycarbonyl-L-2"-propargylglycin-5"-yl)-2',3'-O-isopropylidene-5' $\boldsymbol{O}$-sulfamoyladenosine $(\mathbf{S 1 2 a})$. In a $50 \mathrm{~mL}$ roundbottom flask, 8-alkynyladenosine derivative S11a (115 mg, $0.146 \mathrm{mmol}$, containing 6.7 equiv inseparable DMA) was dissolved in anhyd $\mathrm{EtOH}(4 \mathrm{~mL})$. Solid $5 \% \mathrm{Pd} / \mathrm{C}(60 \mathrm{mg})$ was added and the flask was flushed with $\mathrm{H}_{2}$ gas, then stirred under a balloon of $\mathrm{H}_{2}$ at $\mathrm{rt}$ for $18 \mathrm{~h}$. The mixture was filtered over a celite and concentrated by rotary evaporation. Purification by silica flash chromatography (EtOAc with $0.5 \% \mathrm{AcOH})$ yielded 8-alkyladenosine derivative $\mathbf{S 1 2 a}(100 \mathrm{mg}, 98 \%)$ as a white solid.

TLC: $R_{f} 0.08\left(9: 1 \mathrm{CH}_{2} \mathrm{Cl}_{2}: \mathrm{MeOH}\right)$. IR (NaCl, film): 3251 (N-H st, O-H st), 2979, $1748(\mathrm{C}=\mathrm{O})$, 1713 (C=O st), 1613, 1495, 1368 ( $\mathrm{SO}_{2}$ asym st), 1234, $1149\left(\mathrm{SO}_{2}\right.$ sym st), 1078, 998, 863. ${ }^{1}$ H-NMR $\left(500 \mathrm{MHz}\right.$, acetone- $\left.d_{6}\right): \delta 8.54(\mathrm{~s}, 1 \mathrm{H}), 6.72(\mathrm{~s}, 2 \mathrm{H}), 6.27(\mathrm{~s}, 1 \mathrm{H}), 6.17(\mathrm{~d}, 1 \mathrm{H}, J=7.8)$, $5.76(\mathrm{~d}, 1 \mathrm{H}, J=6.1), 5.30(\mathrm{~m}, 1 \mathrm{H}), 4.45(\mathrm{~d}, 1 \mathrm{H}, J=3.8), 4.3(\mathrm{dd}, 1 \mathrm{H}, J=10.5,6.0), 4.26$ (br, $1 \mathrm{H}), 4.18(\mathrm{dd}, 1 \mathrm{H}, J=10.0,7.0), 3.19(\mathrm{~m}, 1 \mathrm{H}), 3.10(\mathrm{~m}, 1 \mathrm{H}), 1.95(\mathrm{~m}, 2 \mathrm{H}), 1.59,(\mathrm{~s}, 3 \mathrm{H}), 1.53(\mathrm{~s}$, 9H), $1.43-1.38(\mathrm{~m}, 14 \mathrm{H}) ; 2$ exchangeable $\mathrm{H}$ not observed. ${ }^{13} \mathbf{C}-\mathbf{N M R}\left(125 \mathrm{MHz}\right.$, acetone- $\left.d_{6}\right)$ : $\delta 174.4,156.6,156.0,152.7,152.2,151.1,150.1,122.7,114.9,90.5,85.8,84.4,82.9,81.4,79.3$, 69.4, 54.2, 31.9, 30.6, 28.4, 27.9, 27.5, 25.5, 24.3. ESI-MS $m / z$ (rel int): pos $702.4\left([\mathrm{M}+\mathrm{H}]^{+}\right.$, 50), 724.5([M+Na $\left.]^{+}, 100\right)$; neg $700.3\left([\mathrm{M}-\mathrm{H}]^{-}, 100\right)$.

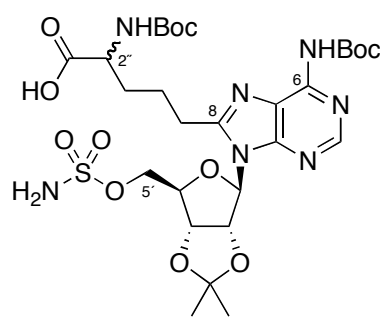

6- $N$-t-Butoxycarbonyl-8-( $N$-t-butoxycarbonyl-DL-2"-propargylglycin-5"-yl)-2, $3^{\prime}-O$-isopropylidene-5'-O -sulfamoyladenosine $(\mathbf{S 1 2 b})$. Prepared from $\mathbf{S 1 1 b}$ as above. White powder (162 mg, 84\%). TLC: $R_{f} 0.08\left(9: 1 \mathrm{CH}_{2} \mathrm{Cl}_{2}: \mathrm{MeOH}\right) . \quad$ IR (NaCl, film): 3250 (N-H st, O-H st), 2981, 1748 (C=O st), 1709 (C=O st), 1612, 1494, 1369 ( $\mathrm{SO}_{2}$ asym st), 1235, 1148 ( $\mathrm{SO}_{2}$ sym st), 1101, 1076, 998, 863. ${ }^{1} \mathrm{H}-\mathbf{N M R}\left(500 \mathrm{MHz}\right.$, acetone- $\left.d_{6}\right): \delta 8.57(\mathrm{~s}, 1 \mathrm{H}), 6.83(\mathrm{~s}, 2 \mathrm{H}), 6.27$ (s, $1 \mathrm{H}), 6.20(\mathrm{~d}, 1 \mathrm{H}, J=8.0), 5.75(\mathrm{~d}, 1 \mathrm{H}, J=6.5), 5.29(\mathrm{~m}, 1 \mathrm{H}), 4.46(\mathrm{~m}, 1 \mathrm{H}), 4.34(\mathrm{~m}, 1 \mathrm{H}), 4.27$ $(\mathrm{m}, 1 \mathrm{H}), 4.20(\mathrm{~m}, 1 \mathrm{H}), 3.17(\mathrm{~m}, 2 \mathrm{H}), 2.0-1.9$ (obsc, $2 \mathrm{H}), 1.59(\mathrm{~m}, 14 \mathrm{H}), 1.41(\mathrm{~m}, 12 \mathrm{H}) ; 2$ exchangeable $\mathrm{H}$ not observed. ${ }^{13} \mathbf{C}$-NMR $\left(125 \mathrm{MHz}\right.$, acetone- $\left.d_{6}\right)$ : $\delta 174.8,174.7^{*}, 156.6,156.24$. $156.21^{*}, 152.7,152.3,151.2,150.0,122.5,114.9,90.5,85.9,84.4^{*}, 84.3$ 82.9, 81.6, 79.4, 69.3, $54.3,31.9,30.4,28.4,27.92,27.88^{*}, 27.48^{*}, 27.46,25.8,25.6^{*}, 24.4 ; 6$ resolved peaks arising specifically from the L-diastereomer S12a above are indicated with a *. ESI-MS $\mathrm{m} / \mathrm{z}$ (rel int): $\operatorname{pos} 702.4\left([\mathrm{M}+\mathrm{H}]^{+}, 60\right), 724.6\left([\mathrm{M}+\mathrm{Na}]^{+}, 100\right)$; neg $700.3\left([\mathrm{M}-\mathrm{H}]^{-}\right)$. 


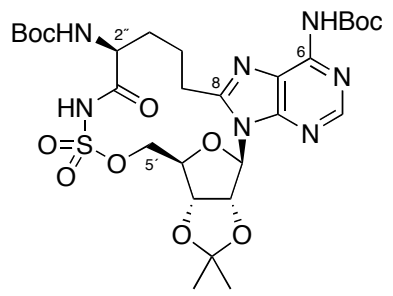

cyclo $^{8 \mathrm{C}_{2} \beta}-6-N$ - $t$-Butoxycarbonyl-5' $-O$ - $(N$-[ $N$ - $t$-butoxycarbonyl-L-alanyl $]$ sulfamoyl)adenosine (S13a). In a $50 \mathrm{~mL}$ recovery flask, 8-alkyladenosine derivative S12a (10.0 $\mathrm{mg}, 14 \mu \mathrm{mol}$, 1.0 equiv) was dissolved in anhyd $\mathrm{CH}_{3} \mathrm{CN}(7 \mathrm{~mL}, 0.002 \mathrm{M})$. HATU $(8.0 \mathrm{mg}, 21 \mu \mathrm{mol}$, 1.5 equiv) and DBU (3.2 $\mu \mathrm{L}, 21 \mu \mathrm{mol}, 1.5$ equiv) were added and the reaction was stirred at $\mathrm{rt}$ for $2 \mathrm{~h}$, then concentrated by rotary evaporation. Purification by silica flash chromatography $\left(20: 1 \rightarrow 15: 1 \quad \mathrm{CH}_{2} \mathrm{Cl}_{2} / \mathrm{MeOH}\right)$ yielded S13a with 1.7 equiv of inseparable DBU (9.0 mg, $9.6 \mu \mathrm{mol}, 68 \%$, corrected for 1.7 equiv DBU).

TLC: $R_{f} 0.21\left(9: 1 \mathrm{CH}_{2} \mathrm{Cl}_{2}: \mathrm{MeOH}\right)$. IR (NaCl, film): 3405 (N-H st), 2979, 1713 (C=O st), 1650 (C=O st), 1614, 1538, 1487, 1371 ( $\mathrm{SO}_{2}$ asym st), 1325, 1248, 1211, 1150 ( $\mathrm{SO}_{2}$ sym st), 1102, 996, 842. ${ }^{1} \mathbf{H}-\mathbf{N M R}\left(500 \mathrm{MHz}, \mathrm{CD}_{3} \mathrm{OD}\right): \delta 8.58(\mathrm{~s}, 1 \mathrm{H}), 6.36(\mathrm{~d}, 1 \mathrm{H}, J=6.9), 5.40(\mathrm{br}, 1 \mathrm{H})$, 5.19 (br, 1H), 4.39 (br, 1H), 4.31 (d, 1H, $J=10.9), 4.10$ (m, 1H, $J=5.1,7.8,3.0), 3.83$ (d, 1H, $J$ $=3.0), 3.15(\mathrm{~m}, 1 \mathrm{H}), 2.83(\mathrm{~m}, 1 \mathrm{H}), 1.90-1.25(\mathrm{~m}, 28 \mathrm{H}) ; 3$ exchangeable $\mathrm{H}$ not observed. ${ }^{13}$ C-NMR (125 MHz, $\left.\mathrm{CD}_{3} \mathrm{OD}\right): \delta 167.5,157.6,157.5,153.5,152.8,152.6,150.2,121.6,116.3$, $90.1,85.5,84.4,83.0,82.1,80.6,70.4,59.2,33.8,30.0,28.8,28.5,27.5,25.7,24.9$. ESI-MS $\mathrm{m} / \mathrm{z}$ (rel int): pos $684.4\left([\mathrm{M}+\mathrm{H}]^{+}, 40\right), 706.4\left([\mathrm{M}+\mathrm{Na}]^{+}, 100\right)$; neg $682.4\left([\mathrm{M}-\mathrm{H}]^{-}, 100\right), 608.2$ $\left([\mathrm{M}+\mathrm{Cl}-\mathrm{Boc}]^{-}, 90\right)$.

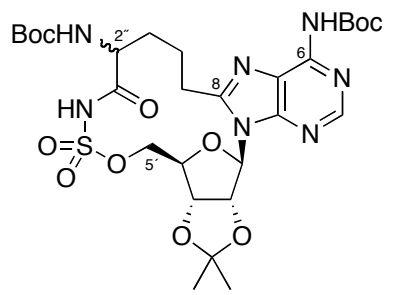

cyclo $^{8 \mathrm{C}_{2} \beta}-6-\mathrm{N}$-t-Butoxycarbonyl-5'-O$-(\mathrm{N}$-[N-t-butoxycarbonyl-DL-alanyl] $\mathbf{s u l f a m o y l})$ adenosine (S13b). Prepared from S12b as above. White powder (38 mg, 48\%, corrected for 2.1 equiv DBU). TLC: $R_{f} 0.21\left(9: 1 \mathrm{CH}_{2} \mathrm{Cl}_{2}: \mathrm{MeOH}\right)$. IR (NaCl, film): $3400(\mathrm{~N}-\mathrm{H}$ st), 2980, 2937, 1704 $(\mathrm{C}=\mathrm{O}$ st $), 1645$ (C=O st), 1601, 1538, 1472, 1385 ( $\mathrm{SO}_{2}$ asym st), 1369, 1324, 1256, $1149\left(\mathrm{SO}_{2}\right.$ sym st), 1077, 977, 838. ${ }^{1} \mathrm{H}-\mathbf{N M R}\left(500 \mathrm{MHz}, \mathrm{CD}_{3} \mathrm{OD}\right): \delta 8.54$ (s, 1H), 6.31 (m, 1H), 5.50 (obsc, $\mathrm{m}, 0.5 \mathrm{H}$, D-stereoisomer), 5.38 (m, 0.5H, L-stereoisomer), $5.23(\mathrm{~m}, 0.5 \mathrm{H}, \mathrm{D}$-stereoisomer $), 5.18$ $(\mathrm{m}, 0.5 \mathrm{H}, \mathrm{L}-$ stereoisomer $), 4.39(\mathrm{~m}, 1 \mathrm{H}), 4.29(\mathrm{~m}, 1 \mathrm{H}), 4.14-4.00(\mathrm{~m}, 1.5 \mathrm{H}), 3.90(\mathrm{~m}, 0.5 \mathrm{H}$, L-stereoisomer), $3.17(\mathrm{~m}, 1 \mathrm{H}), 2.81(\mathrm{~m}, 1 \mathrm{H}), 1.88-1.25(28 \mathrm{H}) ; 3$ exchangeable $\mathrm{H}$ not observed. ${ }^{13}$ C-NMR (125 MHz, $\left.\mathrm{CD}_{3} \mathrm{OD}\right): \delta 167.5,157.8,157.7,153.6,153.4^{*}, 152.8,152.5,150.3$, $150.2^{*}, 121.7,116.3^{*}, 116.0,90.3,90.1^{*}, 85.4,84.7,84.3^{*}, 82.9^{*}, 82.6,82.1,80.5,71.6,58.6$, $33.8,30.0,28.8,28.5,27.5,25.7^{*}, 25.6,24.9 ; 7$ resolved peaks arising specifically from the 
L-diastereomer S13a above are indicated with a *. ESI-MS $m / z$ (rel int): pos $684.1\left([\mathrm{M}+\mathrm{H}]^{+}\right.$, 100), $706.1\left([\mathrm{M}+\mathrm{Na}]^{+}, 95\right)$; neg $682.1\left([\mathrm{M}-\mathrm{H}]^{-}\right)$.

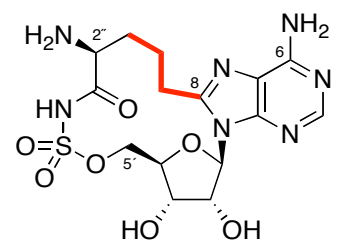

cyclo $^{8 \mathrm{C}_{2} \beta}-5^{\prime}-O$-( $N$-[DL-alanyl] sulfamoyl)adenosine $\left(2 \mathrm{a}\right.$, cyclo $\left.^{8 \mathrm{C}_{2} \beta}-\mathrm{L}-\mathrm{Ala}-\mathrm{AMS}\right) . \quad$ In a $15 \mathrm{~mL}$ roundbottom flask, protected macrocycle S13a $(14.4 \mathrm{mg}, 21 \mu \mathrm{mol}$, corrected for 0.43 equiv DBU) was dissolved in precooled $80 \%$ aq TFA at $0{ }^{\circ} \mathrm{C}$. The reaction was warmed to $\mathrm{rt}$ and stirred for $2 \mathrm{~h}$ when the reaction was complete as judged by LC-MS analysis. MeOH (5 mL) was added and the mixture was concentrated by rotary evaporation and azeotroped from $\mathrm{MeOH}$ three times. Purification by preparative HPLC $\left(\mathrm{C}_{18} ; 250 \times 21.4 \mathrm{~mm}\right.$; gradient $0-20 \% \mathrm{CH}_{3} \mathrm{CN}$ in $\mathrm{H}_{2} \mathrm{O}$ with $0.05 \%$ TFA over $\left.20 \mathrm{~min}, 16 \mathrm{~mL} / \mathrm{min}\right)$ yielded macrocycle $2 \mathbf{a}(9.2 \mathrm{mg}, 99 \%)$ as a white solid.

HPLC: $t_{\text {ret }}=14.0$ min. IR (NaCl, film): $3142(\mathrm{~N}-\mathrm{H}, \mathrm{O}-\mathrm{H} \mathrm{st}), 2954,1684$ (C=O st), 1613, 1519, 1437, 1308, 1196, 1138 ( $\mathrm{SO}_{2}$ sym st), 985, 838. ${ }^{1} \mathbf{H}-\mathbf{N M R}\left(500 \mathrm{MHz}, \mathrm{CD}_{3} \mathrm{OD}\right): \delta 8.30(\mathrm{~s}, 1 \mathrm{H})$, $6.15(\mathrm{~d}, 1 \mathrm{H}, J=3.0), 4.76(\mathrm{t}, 1 \mathrm{H}, J=4.8), 4.47$ (dd, 1H, $J=8.8,7.0), 4.41(\mathrm{dd}, 1 \mathrm{H}, J=11.0$, $1.5), 4.28(\mathrm{dd}, 1 \mathrm{H}, J=11.0,5.0), 4.24(\mathrm{dt}, 1 \mathrm{H}, J=8.0,4.8,1.3), 3.61(\mathrm{dd}, 1 \mathrm{H}, J=8.5,3.0), 3.18$ $(\mathrm{m}, 1 \mathrm{H}), 3.07(\mathrm{~m}, 1 \mathrm{H}), 2.27(\mathrm{~m}, 1 \mathrm{H}), 2.12(\mathrm{~m}, 2 \mathrm{H}), 1.91(\mathrm{~m}, 1 \mathrm{H}) ; 7$ exchangeable $\mathrm{H}$ not observed. ${ }^{13} \mathrm{C}$-NMR $\left(125 \mathrm{MHz}, \mathrm{CD}_{3} \mathrm{OD}\right): \delta$ 175.6, 157.0, 152.3, 151.9, 147.1, 118.5, 89.6, 84.1, 73.5, 71.2, 69.7, 56.7, 31.5, 29.4, 22.4. ESI-MS $m / z$ (rel int): $\operatorname{pos} 444.1\left([\mathrm{M}+\mathrm{H}]^{+}, 95\right), 466.1$ $\left([\mathrm{M}+\mathrm{Na}]^{+}, 100\right) ;$ neg $442.4\left([\mathrm{M}-\mathrm{H}]^{-}, 100\right)$.

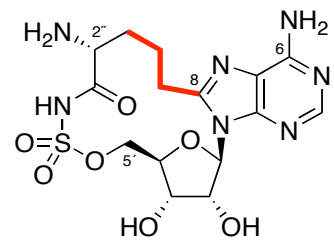

cyclo $^{8 \mathrm{C}_{2} \beta}-5^{\prime}-\mathrm{O}-(\mathrm{N}-[\mathrm{D}-\mathrm{alanyl}] \mathrm{sulfamoyl})$ adenosine (2c, cyclo $\left.^{8 \mathrm{C}_{2} \beta}-\mathrm{D}-\mathrm{Ala}-\mathrm{AMS}\right)$. Prepared from $\mathbf{S 1 3 b}$ as above with isolation of the D-diastereomer by preparative HPLC $\left(\mathrm{C}_{18} ; 250 \times 21.4 \mathrm{~mm}\right.$; gradient $0-20 \% \mathrm{CH}_{3} \mathrm{CN}$ in $\mathrm{H}_{2} \mathrm{O}$ with $0.05 \%$ TFA over $20 \mathrm{~min}, 16 \mathrm{~mL} / \mathrm{min}$ ). White powder ( $1.3 \mathrm{mg}, 40 \%$, versus $50 \%$ theoretical maximum). HPLC: $t_{\mathrm{ret}}=9.5 \mathrm{~min}$. IR (NaCl, film): 3142 (N-H st, O-H st), 2954, 1685 (C=O st), 1619, 1437, 1296, 1202, 1137 (SO sym st), 985, 838. ${ }^{1}$ H-NMR (500MHz, CD $\left.{ }_{3} \mathrm{OD}\right): \delta 8.40(\mathrm{~s}, 1 \mathrm{H}), 6.13(\mathrm{~d}, 1 \mathrm{H}, J=3.85), 4.90($ obsc, $\left.1 \mathrm{H})\right), 4.62(\mathrm{~d}$, $1 \mathrm{H}, J=10.0), 4.58(\mathrm{t}, 1 \mathrm{H}, J=5.7), 4.29(\mathrm{~m}, 2 \mathrm{H}), 3.97(\mathrm{~m}, 1 \mathrm{H}), 3.12(\mathrm{~m}, 1 \mathrm{H}), 3.00(\mathrm{~m}, 1 \mathrm{H}), 2.01$ (m, 4H). 7 exchangeable $\mathrm{H}$ not observed. ${ }^{13} \mathbf{C}-\mathbf{N M R}(125 \mathrm{MHz}): \delta 172.9,157.4,151.3,151.2$, 145.6, 118.6, 90.4, 83.1, 73.7, 73.0, 71.2, 55.4, 29.9, 29.3, 21.8. ESI-MS $m / z$ (rel int): pos 444.1 $\left([\mathrm{M}+\mathrm{H}]^{+}, 95\right), 466.1\left([\mathrm{M}+\mathrm{Na}]^{+}, 100\right) ;$ neg $422.5\left([\mathrm{M}-\mathrm{H}]^{-}\right)$. 


\section{F. SYNTHESIS OF $C Y C L O^{8 \mathrm{C} 3}{ }_{3}$-ALA-AMS MACROCYCLES $(2 \mathrm{~b}, 2 \mathrm{~d})$}

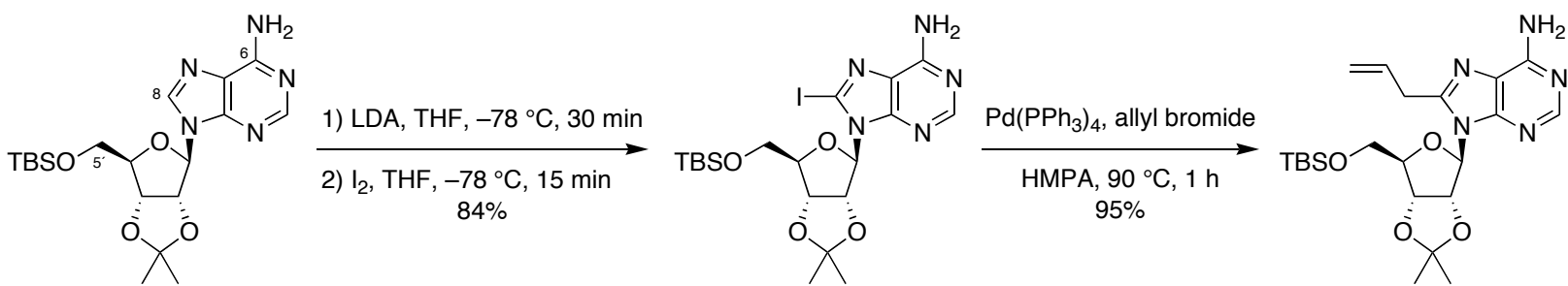

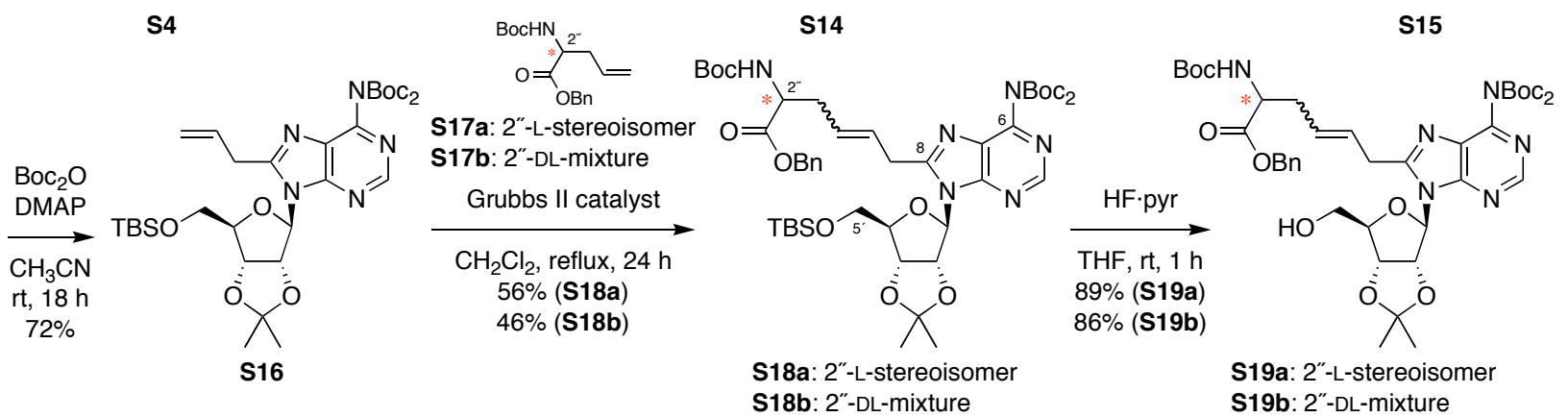

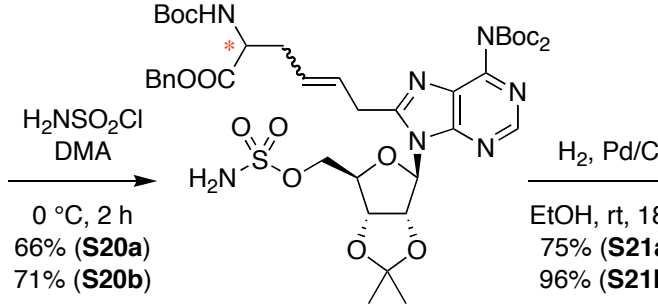

S20a: 2 "-L-stereoisomer S20b: 2"-DL-mixture<smiles>Nc1ncnc2c1nc(CCCC[C@H](N)C(=O)NS(=O)(=O)O)n2[C@@H]1O[C@H](CO[C@H]2[C@@H](O)[C@H]2O)[C@H](O)[C@H]1O</smiles>

2b: 2"-L-stereoisomer

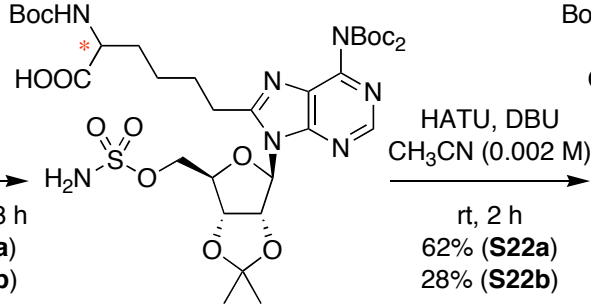

S21a: 2"-L-stereoisomer S21b: 2"-DL-mixture<smiles>C[C@H](N)C(=O)NS(=O)(=O)OC[C@H]1O[C@H](n2c(CCCC[C@H](N)C(N)=O)nc3c(N)ncnc32)[C@@H](O)[C@@H]1O</smiles>

2d: 2"-D-stereoisomer

Figure S5. Synthesis of macrocycles $\mathbf{2 b}$ and $\mathbf{2 d}$ having a 3-carbon linker (red). Macrocycles $\mathbf{2 b}$ and 2d were synthesized from the known adenosine acetonide S4. ${ }^{14}$ Cross metathesis of S16 and S17 yielded $\mathbf{S 1 8}$ as a 2:1 mixture of inseparable olefin stereoisomers whose configurations could not be determined unambiguously. These compounds were carried together through the synthesis to S20, at which point the olefin was reduced to afford a single compound S21a. The D-stereoisomer 2d was obtained by synthesis using racemic allyl glycine $\mathbf{S 1 7 b}$, followed by HPLC separation of the diasteromers at the conclusion of the synthesis. (DMAP $=4$-(dimethylamino)pyridine; HMPA $=$ hexamethylphosphoramide). 


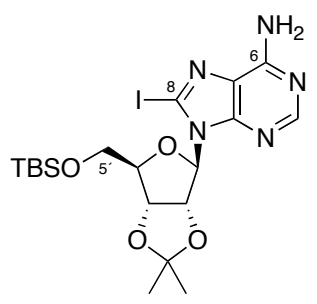

$5^{\prime}-\boldsymbol{O}$ - $t$-Butyldimethylsilyl-8-iodo-2',3'-O -isopropylideneadenosine (S14). In a $200 \mathrm{~mL}$ roundbottom flask, $i$ - $\operatorname{Pr}_{2} \mathrm{NH}(5.3 \mathrm{~mL}, 37.4 \mathrm{mmol}, 4.48$ equiv) was dissolved in THF $(20 \mathrm{~mL})$ and cooled to $-78{ }^{\circ} \mathrm{C}$. $n$-BuLi $(2.5 \mathrm{M}$ in hexanes, $14.5 \mathrm{~mL}, 36.4 \mathrm{mmol}, 4.36$ equiv) was added and the mixture was stirred for $30 \mathrm{~min}$. Adenosine derivative $\mathbf{S 4}{ }^{14}$ (3.51 g, 8.34 mmol, 1.0 equiv) was azeotroped from toluene, then dissolved in THF $(17 \mathrm{~mL})$ and added to the LDA solution via cannula. After stirring at $-78{ }^{\circ} \mathrm{C}$ for $30 \mathrm{~min}$, a solution of $\mathrm{I}_{2}(5.2 \mathrm{~g}, 20.8 \mathrm{mmol}, 2.49$ equiv) in THF $(10 \mathrm{~mL})$ was added via cannula over $5 \mathrm{~min}$. The resulting deep purple solution was stirred at $-78^{\circ} \mathrm{C}$ for an additional $10 \mathrm{~min}$. The reaction was quenched by addition of satd aq $\mathrm{NH}_{4} \mathrm{Cl}$ $(10 \mathrm{~mL})$ and satd aq $\mathrm{Na}_{2} \mathrm{~S}_{2} \mathrm{O}_{3}(30 \mathrm{~mL})$ and extracted with EtOAc $(3 \times 100 \mathrm{~mL})$. The combined organic extracts were washed with $\mathrm{NH}_{4} \mathrm{Cl}(2 \times 50 \mathrm{~mL})$ and $\mathrm{NaCl}(2 \times 50 \mathrm{~mL})$, dried $\left(\mathrm{Mg}_{2} \mathrm{SO}_{4}\right)$, filtered, and concentrated by rotary evaporation. Purification by silica flash chromatography (5:4 hexanes/EtOAc) yielded 8-iodoadenosine S14 (3.81 g, 84\%) as a yellow solid.

TLC: $R_{f} 0.26$ (1:1 hexanes/EtOAc). IR (NaCl, film): $3300(\mathrm{~N}-\mathrm{H}$ st), 2857, 1640, 1595, 1472, 1441, 1412, 1373, 1316, 1290, 1261, 1232, 1210, 1156, 1083, 1005, 908, 836. ${ }^{1} \mathbf{H}-\mathbf{N M R}$ $\left(500 \mathrm{MHz}, \mathrm{CDCl}_{3}\right): \delta 8.20(\mathrm{~s}, 1 \mathrm{H}), 6.29(\mathrm{~s}, 2 \mathrm{H}), 6.07(\mathrm{~d}, 1 \mathrm{H}, J=1.8), 5.81(\mathrm{~m}, 1 \mathrm{H}), 5.15(\mathrm{dd}$, $1 \mathrm{H}, J=6.2,3.2), 4.28(\mathrm{ddd}, 1 \mathrm{H}, J=9.8,6.6,3.2), 3.73(\mathrm{dd}, 1 \mathrm{H}, J=10.6,6.8), 3.62(\mathrm{dd}, 1 \mathrm{H}, J=$ 10.6, 6.5), $1.61(\mathrm{~s}, 3 \mathrm{H}), 1.39(\mathrm{~s}, 3 \mathrm{H}), 0.81(\mathrm{~s}, 9 \mathrm{H}),-0.07(\mathrm{~d}, 6 \mathrm{H}, J=5.0) .{ }^{13} \mathbf{C}-\mathbf{N M R}(125 \mathrm{MHz}$, $\left.\mathrm{CDCl}_{3}\right): \delta 154.3,152.7,150.4,122.8,113.9,100.6,93.5,88.3,82.8,83.3,63.2,27.2,25.9,25.5$, 18.4, -5.3, -5.4. ESI-MS $m / z$ (rel int): $\operatorname{pos} 547.8\left([\mathrm{M}+\mathrm{H}]^{+}, 100\right), 569.9\left([\mathrm{M}+\mathrm{Na}]^{+}, 10\right)$; neg $545.9\left([\mathrm{M}-\mathrm{H}]^{-}, 100\right), 591.7\left([\mathrm{M}+\mathrm{Cl}]^{-}, 59\right)$.

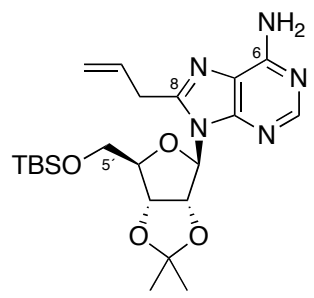

8-Allyl-5' $\boldsymbol{O}$-t-butyldimethylsilyl-2',3'-O -isopropylideneadenosine (S15). In a $100 \mathrm{~mL}$ roundbottom flask, 8-iodoadenosine S14 (1.16 g, $2.12 \mathrm{mmol}, 1.0$ equiv) was dissolved in anhyd HMPA (10 mL). Allyltributyltin ( $1.0 \mathrm{~mL}, 3.2 \mathrm{mmol}, 1.5$ equiv) and $\mathrm{Pd}\left(\mathrm{PPh}_{3}\right)_{4}(485 \mathrm{mg}$, $0.42 \mathrm{mmol}, 0.2$ equiv $)^{16}$ were added and the mixture was heated to $90{ }^{\circ} \mathrm{C}$ for $1 \mathrm{~h}$. The mixture was cooled to $\mathrm{rt}$ and diluted with satd aq $\mathrm{NH}_{4} \mathrm{Cl}(50 \mathrm{~mL})$, then extracted with EtOAc $(2 \times 100 \mathrm{~mL})$. The combined organic extracts were washed with satd $\mathrm{NH}_{4} \mathrm{Cl}(3 \times 50 \mathrm{~mL})$, satd aq $\mathrm{NaHCO}_{3}(3 \times 50 \mathrm{~mL})$, and brine $(3 \times 50 \mathrm{~mL})$, dried $\left(\mathrm{MgSO}_{4}\right)$, filtered, and concentrated by rotary evaporation. Purification by silica flash chromatography (4:5 hexanes/EtOAc) yielded 8-allyladenosine $\mathbf{S 1 5}$ (929 $\mathrm{mg}$, 95\%) as a yellow solid. 
TLC: $R_{f} 0.10$ (1:1 hexanes/EtOAc). IR (NaCl, film): 3317 (N-H st), 3164 (N-H st), 2930, 2856, 1644, 1602, 1472, 1436, 1372, 1329, 1297, 1256, 1212, 1157, 1006, 920, 837. ${ }^{1}$ H-NMR $\left(500 \mathrm{MHz}, \mathrm{DMSO}-d_{6}\right): \delta 8.11(\mathrm{~s}, 1 \mathrm{H}), 7.22(\mathrm{~s}, 2 \mathrm{H}), 6.07(\mathrm{~m}, 2 \mathrm{H}), 5.74(\mathrm{dd}, 1 \mathrm{H}, J=6.1,1.5)$, $5.16(\mathrm{~m}, 2 \mathrm{H}), 5.06(\mathrm{dd}, 1 \mathrm{H}, J=6.2,3.1), 4.11(\mathrm{~m}, 1 \mathrm{H}), 3.80-3.63(\mathrm{~m}, 3 \mathrm{H}), 3.56(\mathrm{~m}, 1 \mathrm{H}), 1.53(\mathrm{~s}$, $3 \mathrm{H}), 1.33(\mathrm{~s}, 3 \mathrm{H}), 0.80(\mathrm{~s}, 9 \mathrm{H}),-0.13(\mathrm{~s}, 6 \mathrm{H}) .{ }^{13} \mathbf{C}-\mathbf{N M R}\left(125 \mathrm{MHz}, \mathrm{DMSO}-d_{6}\right): \delta$ 157.1, 153.7, 151.6, 151.4, 134.9, 119.6, 114.8, 90.6, 88.8, 84.1, 83.2, 64.6, 33.6, 32.4, 28.7, 27.4, 27.0, 19.7, $-3.77,-3.86$. ESI-MS $\mathrm{m} / \mathrm{z}$ (rel int): pos $462.01\left([\mathrm{M}+\mathrm{H}]^{+}, 100\right)$; neg $460.01\left([\mathrm{M}-\mathrm{H}]^{-}, 100\right)$, $505.89\left([\mathrm{M}+\mathrm{Cl}]^{-}, 30\right)$.

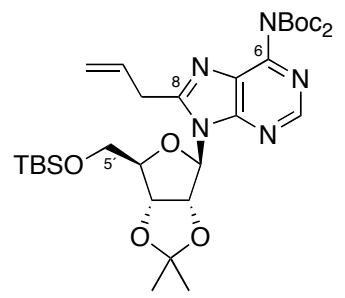

8-Allyl-6- $N$-bis(t-butoxycarbonyl)-5'-O-t-butyldimethylsilyl-2', $3^{\prime}-O$-isopropylideneadenosine (S16). In a $100 \mathrm{~mL}$ roundbottom flask, 8-allyladenosine $\mathbf{S 1 5}(850 \mathrm{mg}, 1.84 \mathrm{mmol}$, 1.0 equiv) was dissolved in THF ( $18 \mathrm{~mL})$. DMAP $(45 \mathrm{mg}, 0.368 \mathrm{mmol}, 0.2$ equiv) was added and the mixture was cooled to $0{ }^{\circ} \mathrm{C}$. Boc $_{2} \mathrm{O}(1.0 \mathrm{~g}, 4.6 \mathrm{mmol}, 2.5$ equiv) was added and the mixture was stirred at $0{ }^{\circ} \mathrm{C}$ for $5 \mathrm{~min}$, then removed from the ice bath and allowed to warm to rt. After stirring for $18 \mathrm{~h}$, the mixture was concentrated by rotary evaporation. Purification by silica flash chromatography (9:2 hexanes/EtOAc) yielded 8-allyl-6- $N$-bis(Boc)adenosine $\mathbf{S 1 6}$ (874 mg, $72 \%)$ as a clear foam.

TLC: $R_{f} 0.87$ (1:1 hexanes/EtOAc). IR ( $\mathrm{NaCl}$, film): 2930, 2856, 1644 (C=O st), 1602, 1472, 1436, 1372, 1329, 1297, 1256, 1212, 1006, 837, 920, 837. ${ }^{1} \mathbf{H}-\mathbf{N M R}\left(400 \mathrm{MHz}, \mathrm{CDCl}_{3}\right): \delta 8.76$ (s, 1H), $6.08(\mathrm{~m}, 2 \mathrm{H}), 5.78(\mathrm{dd}, 1 \mathrm{H}, J=5.2,2.3), 5.25(\mathrm{~m}, 2 \mathrm{H}), 5.13(\mathrm{dd}, 1 \mathrm{H}, J=4.6,3.4), 4.26$ $(\mathrm{m}, 1 \mathrm{H}), 3.81(\mathrm{~d}, 2 \mathrm{H}, J=6.3), 2.95(\mathrm{~m}, 2 \mathrm{H}), 1.59(\mathrm{~s}, 3 \mathrm{H}), 1.43(\mathrm{~m}, 21 \mathrm{H}), 0.86(\mathrm{~s}, 9 \mathrm{H}),-0.06(\mathrm{~s}$, 6H). ${ }^{13}$ C-NMR $\left(100 \mathrm{MHz}, \mathrm{CDCl}_{3}\right): \delta 155.6,153.7,151.2,150.3,149.4,131.3,128.3,118.9$, $114.2,89.9,87.5,83.6,82.8,81.8,63.0,32.9,27.8,27.2,25.8,25.4,18.3,-5.40,-5.44$. ESI-MS $\mathrm{m} / \mathrm{z}$ (rel int): pos $662.0\left([\mathrm{M}+\mathrm{H}]^{+}, 41\right), 684.0\left([\mathrm{M}+\mathrm{Na}]^{+}, 100\right)$; neg $660.0\left([\mathrm{M}-\mathrm{H}]^{-}, 100\right), 696.2$ $\left([\mathrm{M}+\mathrm{Cl}]^{-}, 15\right)$.

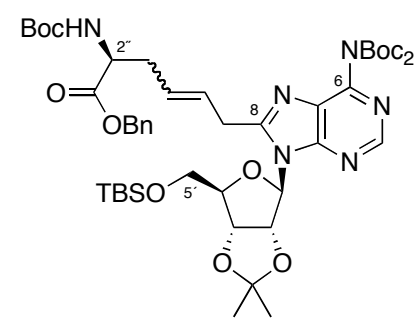

6- $N$-Bis(t-butoxycarbonyl)-8-(O-benzyl- $N$-[ $t$-butoxycarbonyl]-L-2"-E/Z-crotylglycin-6"-yl)$5^{\prime}-O$ - $t$-butyldimethylsilyl-2',3'-O -isopropylideneadenosine (S18a). In a $50 \mathrm{~mL}$ roundbottom 
flask, 8-allyladenosine $\mathbf{S 1 6}$ (207 mg, $0.314 \mathrm{mmol}, 1.0$ equiv) and $O$-Bn- $N$-Boc-L-2-allylglycine ${ }^{19}$ S17a (350 mg, $1.1 \mathrm{mmol}, 3.5$ equiv) were dissolved in $\mathrm{CH}_{2} \mathrm{Cl}_{2}$ (2 mL). Benzylidene[1,3-bis(2,4,6-trimethylphenyl)-2-imidazolidinylidene]dichloro(tricyclohexylphosphine)ruthenium

(Grubbs II catalyst) (26 mg, $0.031 \mathrm{mmol}, 0.10$ equiv) was added and the mixture was heated to reflux with stirring for $12 \mathrm{~h}$. Additional Grubbs II catalyst was added (26 mg, $0.031 \mathrm{mmol}$, 0.10 equiv) and the reaction stirred at reflux for an additional $12 \mathrm{~h}$. The mixture was then concentrated by rotary evaporation. Purification by silica flash chromatography $(9: 2 \rightarrow 3: 1$ hexanes/EtOAc) yielded 8-allyladenosine derivative S18a (163 mg, 56\%) as a red solid comprised of a 2:1 mixture of olefin stereoisomers (configurations could not be determined unambiguously).

TLC: $R_{f} 0.66$ (1:1 hexanes/EtOAc). IR (NaCl, film): 2932, $1790(\mathrm{C}=\mathrm{O}$ st), $1716(\mathrm{C}=\mathrm{O}$ st), 1603, 1579, 1503, 1455, 1369, 1344, 1253, 1213, 1161, 1103, 968. ${ }^{1}$ H-NMR $\left(500 \mathrm{MHz}, \mathrm{C}_{6} \mathrm{D}_{6}\right)$ :

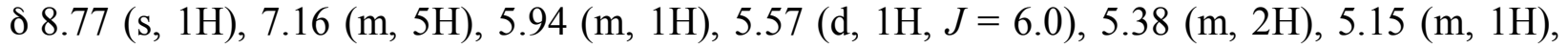
$4.97(\mathrm{~d}, 1 \mathrm{H}, J=12.5), 4.79(\mathrm{~d}, 1 \mathrm{H}, J=12.2), 4.58(\mathrm{~m}, 2 \mathrm{H}), 3.68(\mathrm{~m}, 2 \mathrm{H}), 3.17(\mathrm{~m}, 2 \mathrm{H}), 2.38(\mathrm{~m}$, $1 \mathrm{H}), 2.28(\mathrm{~m}, 1 \mathrm{H}), 1.40-1.20(\mathrm{~m}, 33 \mathrm{H}), 0.90(\mathrm{~s}, 9 \mathrm{H}),-0.09(\mathrm{~s}, 6 \mathrm{H}) ; 1$ exchangeable $\mathrm{H}$ not observed. ${ }^{13}$ C-NMR $\left(125 \mathrm{MHz}, \mathrm{C}_{6} \mathrm{D}_{6}\right)$ : $\delta$ 169.6, 153.3, 153.1, 151.8, 149.3, 149.2, 148.5, 134.1, 127.1-125.5 (obsc, 4C), 111.8, 87.9, 86.9, 81.9, 81.7, 81.0, 80.9, 80.8, 77.5, 64.8, 61.5, 51.6, 33.3, 29.8, 26.3, 25.7, 23.5, 23.3, 20.9, 12.2, -7.3, -7.4. ESI-MS $m / z$ (rel int): pos 939.6 $\left([\mathrm{M}+\mathrm{H}]^{+}, 40\right), 961.7\left([\mathrm{M}+\mathrm{Na}]^{+}, 100\right)$; neg $937.9\left([\mathrm{M}-\mathrm{H}]^{-}, 100\right), 973.8\left([\mathrm{M}+\mathrm{Cl}]^{-}, 80\right)$.

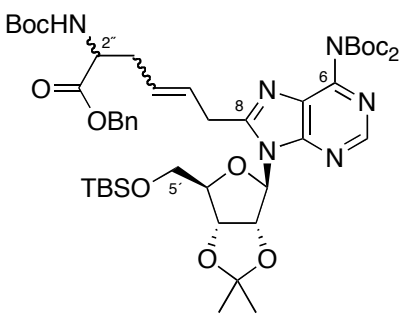

6- $N$-Bis( $t$-butoxycarbonyl)-8-(O-benzyl- $N$-[ $t$-butoxycarbonyl]-DL-2"-E/Z-crotylglycin-6"-yl)$5^{\prime}$ - $O$-t-butyldimethylsilyl-2',3'-O $\boldsymbol{O}$-isopropylideneadenosine $(\mathbf{S 1 8 b})$. Prepared from $O$-Bn- $N$ Boc-DL-2-allylglycine S17b as above as a 2:1 mixture of olefin stereoisomers (configurations could not be determined unambiguously. White powder $(180 \mathrm{mg}, 46 \%)$. TLC: $R_{f} 0.66(1: 1$ hexanes/EtOAc). IR ( $\mathrm{NaCl}$, film): 2979, 1790 (C=O st), 1715 (C=O st), 1604, 1580, 1504, 1455, 1369, 1344, 1253, 1213, 1161, 1104, 969. ${ }^{1} \mathbf{H}-\mathbf{N M R}\left(400 \mathrm{MHz}, \mathrm{C}_{6} \mathrm{D}_{6}\right): \delta 8.77(\mathrm{~s}, 1 \mathrm{H})$, $7.14(\mathrm{~m}, 5 \mathrm{H}) 5.94(\mathrm{~s}, 1 \mathrm{H}), 5.58(\mathrm{~m}, 1 \mathrm{H}), 5.40(\mathrm{~m}, 2 \mathrm{H}), 5.16(\mathrm{~m}, 1 \mathrm{H}), 5.00(\mathrm{~m}, 1 \mathrm{H}), 4.81(\mathrm{~m}, 1 \mathrm{H})$, $4.56(\mathrm{~m}, 2 \mathrm{H}), 3.70(\mathrm{~m}, 2 \mathrm{H}), 3.16(\mathrm{~m}, 2 \mathrm{H}), 2.41(\mathrm{~m}, 1 \mathrm{H}), 2.28(\mathrm{~m}, 1 \mathrm{H}), 1.61(\mathrm{~m}, 3 \mathrm{H}), 1.41(\mathrm{~m}$, 27H), 1.27, $(\mathrm{m}, 3 \mathrm{H}), 0.87(\mathrm{~m}, 9 \mathrm{H}),-0.07(\mathrm{~m}, 6 \mathrm{H}) ; 1$ exchangeable $\mathrm{H}$ not observed. ${ }^{13} \mathbf{C}-\mathbf{N M R}$ $\left(100 \mathrm{MHz}, \mathrm{C}_{6} \mathrm{D}_{6}\right): \delta 169.6,153.4,153.2,151.8,149.4,149.3,148.5,134.1,127.1-125.5$ (obsc, 4C), 111.9, 111.7*, 88.1, 88.0*, 87.0, 81.9, 81.5, 81.1, 80.9, 80.8, 77.6, 65.0, 64.9*, 61.6, 61.5*, $51.7,33.4,29.9,26.4,25.8,23.9,23.5^{*}, 23.4,21.0,12.3,-7.2,-7.3 ; 5$ resolved peaks arising specifically from the L-diastereomer S18a above are indicated with a *. ESI-MS $\mathrm{m} / \mathrm{z}$ (rel int): pos $939.6\left([\mathrm{M}+\mathrm{H}]^{+}, 80\right), 961.7\left([\mathrm{M}+\mathrm{Na}]^{+}, 100\right)$; neg $937.7\left([\mathrm{M}-\mathrm{H}]^{-}, 65\right), 973.8\left([\mathrm{M}+\mathrm{Cl}]^{-}, 100\right)$.

\footnotetext{
${ }^{19}$ Sakaitani, M.; Ohfune, Y. J. Org. Chem. 1990, 55, 870-876.
} 


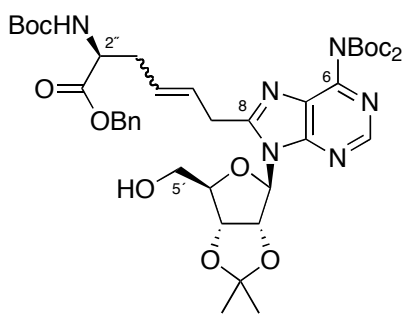

6- $N$-Bis(t-butoxycarbonyl)-8-( $O$-benzyl- $N$-[t-butoxycarbonyl]-L-2"-E/Z-crotylglycin-6"-yl)$\mathbf{2}^{\prime}, \mathbf{3}^{\prime} \mathbf{- O}$-isopropylideneadenosine $(\mathbf{S 1 9 a})$. In a $50 \mathrm{~mL}$ plastic Falcon tube, 5 - $O$-TBS-adenosine S18a (152 mg, $0.162 \mathrm{mmol}, 1.0$ equiv) was dissolved in THF (1.6 mL). HF.pyr (80 $\mu \mathrm{L}$, $4.5 \mathrm{mmol}, 27$ equiv) was added dropwise over $1 \mathrm{~min}$ and the reaction was stirred at $\mathrm{rt}$ for $30 \mathrm{~min}$. A second aliquot of HF.pyr (50 $\mu \mathrm{L}, 2.73 \mathrm{mmol}, 17$ equiv) was added and the reaction was stirred at $\mathrm{rt}$ for an additional $40 \mathrm{~min}$. The reaction was quenched by addition of cold $1 \mathrm{~N} \mathrm{NaOH}$ $(10 \mathrm{~mL})$, then diluted with brine $(30 \mathrm{~mL})$, and extracted with EtOAc $(3 \times 70 \mathrm{~mL})$. The combined organic extracts were washed with $1 \mathrm{~N} \mathrm{HCl}(3 \times 20 \mathrm{~mL})$, dried $\left(\mathrm{MgSO}_{4}\right)$, filtered, and concentrated by rotary evaporation. Purification by silica flash chromatography (3:2 hexanes/EtOAc) yielded alcohol S19a $(119 \mathrm{mg}, 89 \%)$ as a white solid comprised of a 2:1 mixture of olefin stereoisomers (configurations could not be determined unambiguously).

TLC: $R_{f} 0.12$ (1:1 hexanes/EtOAc). IR ( $\mathrm{NaCl}$, film): 2979, 1789 (C=O st), 1715 (C=O st), 1605, 1580, 1504, 1455, 1369, 1344, 1253, 1213, 1161, 1104, 969. ${ }^{1}$ H-NMR (500 MHz, acetone-d $\left.{ }_{6}\right)$ : $\delta 8.80(\mathrm{~s}, 1 \mathrm{H}), 7.34(\mathrm{~m}, 5 \mathrm{H}), 6.10(\mathrm{~s}, 1 \mathrm{H}), 5.71(\mathrm{~m}, 2 \mathrm{H}), 5.47-5.09(\mathrm{~m}, 7 \mathrm{H}), 4.53-4.20(\mathrm{~m}, 4 \mathrm{H})$, 3.71 (br, 2H), 2.53 (br, 2H), $1.63(\mathrm{~s}, 3 \mathrm{H}), 1.42(\mathrm{~m}, 30 \mathrm{H}),{ }^{13} \mathbf{C}-\mathbf{N M R}\left(125 \mathrm{MHz}\right.$, acetone- $\left.d_{6}\right)$ : $\delta 172.5,156.8,156.2,154.5,151.6,151.2,150.4,137.2,129.7,129.3,129.1,129.0,128.9,128.1$, $114.8,91.8,87.7,84.0,83.8,82.5,79.5,67.1,63.2,54.6,35.6,32.6,28.6,27.9,27.8,25.6$. ESI-MS $m / z$ (rel int): pos $825.5\left([\mathrm{M}+\mathrm{H}]^{+}, 20\right), 847.4\left([\mathrm{M}+\mathrm{Na}]^{+}, 100\right)$; neg $823.7\left([\mathrm{M}-\mathrm{H}]^{-}, 20\right)$, $859.6\left([\mathrm{M}+\mathrm{Cl}]^{-}, 100\right)$.

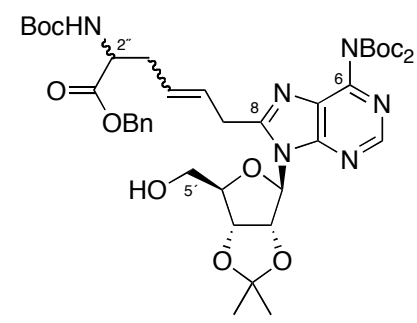

6- $N$-Bis(t-butoxycarbonyl)-8-( $O$-benzyl- $N$-[t-butoxycarbonyl]-DL-2"-E/Z-crotylglycin-6"-yl)$\mathbf{2}^{\prime}, \mathbf{3}^{\prime}$ - $\boldsymbol{O}$-isopropylideneadenosine (S19b). Prepared from S18b as above as a 2:1 mixture of olefin stereoisomers (configurations could not be determined unambiguously). White powder (353 mg, 86\%). TLC: $R_{f} 0.12$ (1:1 hexanes/EtOAc). IR (NaCl, film): 3348 (N-H st, O-H st), 2980, 1790 (C=O st), 1714 (C=O st), 1605, 1504, 1454, 1369, 1253, 1162, 1105, 853. ${ }^{1} \mathbf{H}-\mathbf{N M R}$ $\left(500 \mathrm{MHz}\right.$, acetone- $\left.d_{6}\right): \delta 8.81(\mathrm{~m}, 1 \mathrm{H}), 7.34(\mathrm{~m}, 5 \mathrm{H}), 6.10(\mathrm{~s}, 0.5 \mathrm{H}, \mathrm{L}-$-stereoismer $), 5.95(\mathrm{~m}$, 0.5H, D-stereoismer), $5.50(\mathrm{~m}, 2 \mathrm{H}), 5.44-4.87(\mathrm{~m}, 7 \mathrm{H}), 4.52-4.17(\mathrm{~m}, 4 \mathrm{H}), 3.70(\mathrm{br}, 2 \mathrm{H}), 2.53$ (br, 2H), $1.65(\mathrm{~m}, 3 \mathrm{H}), 1.40(\mathrm{~m}, 30 \mathrm{H}) .{ }^{13} \mathrm{C}-\mathrm{NMR}(125 \mathrm{MHz}): \delta 172.6,172.5^{*}, 157.0,156.7^{*}$, 156.3 , 154.5, 151.6, 151.1, 150.3, 137.1, 129.7, 129.3, 129.2, 129.1, 129.0, 128.1*, 128.0, 
114.82*, 114.77, 91.8*, 91.7, 87.72*, 87.68, 84.0, 83.7, 82.52*, 82.47, 79.5, 67.11*, 67.07, 63.18*, 63.05, 54.6*, 54.5, 35.6, 32.2*, 32.0, 28.5, 27.9, 27.8*, 27.7, 25.62*, 25.56; 13 resolved peaks arising specifically from the L-diastereomer S19a above are indicated with a *. ESI-MS $\mathrm{m} / \mathrm{z}$ (rel int): pos $825.6\left([\mathrm{M}+\mathrm{H}]^{+}, 50\right), 847.5\left([\mathrm{M}+\mathrm{Na}]^{+}, 100\right)$; neg $823.7\left([\mathrm{M}-\mathrm{H}]^{-}, 10\right), 859.7$ $\left([\mathrm{M}+\mathrm{Cl}]^{-}, 100\right)$.

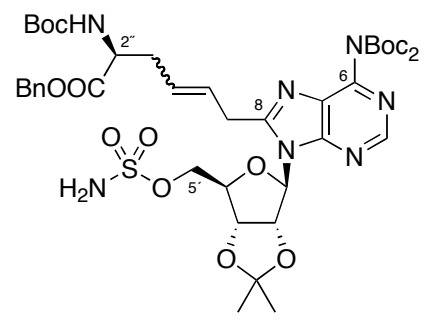

6- $N$-Bis(t-butoxycarbonyl)-8-( $O$-benzyl- $N$-[t-butoxycarbonyl]-L-2"-E/Z-crotylglycin-6"-yl)$2^{\prime}, 3^{\prime}-O$-isopropylidene-5'-O-sulfamoyladenosine (S20a). Prepared by sulfamoylation of alcohol S19a (109 mg, $0.132 \mathrm{mmol}$ ) as described for S12a above. Purification by silica flash chromatography (3:2 hexanes/EtOAc) yielded 5'-O-sulfamoyladenosine S20a $(78.6 \mathrm{mg}, 66 \%$, corrected for 2.5 equiv inseparable DMA) as a white solid comprised of a 2:1 mixture of olefin stereoisomers (configurations could not be determined unambiguously).

TLC: $R_{f} 0.12$ (1:1 hexanes/EtOAc). IR (NaCl, film): 3372 (N-H st), 2979, 1790 (C=O st), 1715 $(\mathrm{C}=\mathrm{O}$ st $), 1605,1512,1455,1369$ ( $\mathrm{SO}_{2}$ asym st $), 1253,1160\left(\mathrm{SO}_{2}\right.$ sym st $), 1107,1000,853$. ${ }^{1}$ H-NMR $\left(500 \mathrm{MHz}\right.$, acetone- $\left.d_{6}\right)$ : $\delta 8.75(\mathrm{~s}, 1 \mathrm{H}), 7.37(\mathrm{~m}, 5 \mathrm{H}), 6.70(\mathrm{~s}, 2 \mathrm{H}), 6.26(\mathrm{~m}, 2 \mathrm{H}), 5.73$ $(\mathrm{m}, 1 \mathrm{H}), 5.66(\mathrm{~s}, 2 \mathrm{H}), 5.32(\mathrm{~m}, 1 \mathrm{H}), 5.17(\mathrm{~m}, 2 \mathrm{H}), 4.49(\mathrm{~m}, 1 \mathrm{H}), 4.39-4.22(\mathrm{~m}, 3 \mathrm{H}), 3.85(\mathrm{~m}$, 2H), $2.55(\mathrm{~m}, 2 \mathrm{H}), 1.61(\mathrm{~s}, 3 \mathrm{H}), 1.44(\mathrm{~s}, 18 \mathrm{H}), 1.37(\mathrm{~m}, 12 \mathrm{H}) .{ }^{13} \mathbf{C}-\mathbf{N M R}(125 \mathrm{MHz}): \delta 172.3$, 156.6, 156.1, 155.0, 151.7, 151.1, 149.9, 137.0, 129.6, 129.1, 129.0, 128.8, 128.4, 127.9, 114.9, 90.4, 85.5, 84.3, 83.8, 82.5, 79.3, 69.1, 66.9, 54.4, 35.4, 31.9, 28.4, 27.7, 27.3, 25.4. ESI-MS $\mathrm{m} / \mathrm{z}$ (rel int): pos $904.3\left([\mathrm{M}+\mathrm{H}]^{+}, 10\right), 926.5\left([\mathrm{M}+\mathrm{Na}]^{+}, 100\right)$; neg $902.6\left([\mathrm{M}-\mathrm{H}]^{-}, 100\right), 938.7$ $\left([\mathrm{M}+\mathrm{Cl}]^{-}, 35\right)$.

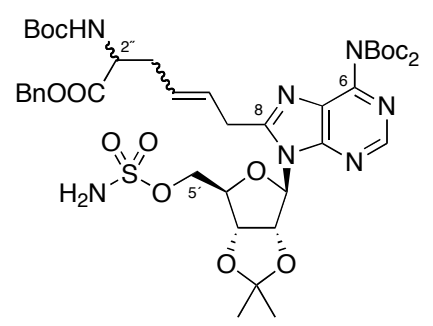

6- $N$-Bis( $t$-butoxycarbonyl)-8-( $O$-benzyl- $N$-[t-butoxycarbonyl]-DL-2"-E/Z-crotylglycin-6"-yl)$\mathbf{2}^{\prime}, 3^{\prime}-\boldsymbol{O}$-isopropylidene-5'-O-sulfamoyladenosine $(\mathbf{S 2 0 b})$. Prepared from $\mathbf{S 1 9 b}$ as above as a 2:1 mixture of olefin stereoisomers (configurations could not be determined unambiguously). Foamy clear solid (316 mg, $0.321 \mathrm{mmol}, 71 \%$; corrected for 1.4 equiv inseparable DMA). TLC: $R_{f} 0.12(1: 1$ hexanes/EtOAc). IR ( NaCl, film): $3356(\mathrm{~N}-\mathrm{H}$ st), 2980, $1788(\mathrm{C}=\mathrm{O}$ st), $1714(\mathrm{C}=\mathrm{O}$ st), 1605, 1513, 1454, 1369 ( $\mathrm{SO}_{2}$ asym st), 1253, 1161 ( $\mathrm{SO}_{2}$ sym st), 1107, 1000, 852. ${ }^{1}$ H-NMR $\left(500 \mathrm{MHz}\right.$, acetone- $\left.d_{6}\right): \delta 8.75(\mathrm{~s}, 1 \mathrm{H}), 7.36(\mathrm{~m}, 5 \mathrm{H}), 6.71(\mathrm{~s}, 2 \mathrm{H}), 6.27(\mathrm{~m}, 2 \mathrm{H}), 5.73(\mathrm{~m}, 1 \mathrm{H})$, 
$5.66(\mathrm{~m}, 2 \mathrm{H}), 5.31(\mathrm{~m}, 1 \mathrm{H}), 5.20-5.13(\mathrm{~m}, 2 \mathrm{H}), 4.49(\mathrm{~m}, 1 \mathrm{H}), 4.38(\mathrm{~m}, 1 \mathrm{H}), 4.24(\mathrm{~m}, 2 \mathrm{H}), 3.86$ (m, 2H), 2.55 (m, 2H), 1.59 (s, 3H), 1.44-1.36 (m, 30H). ${ }^{13} \mathbf{C}-\mathbf{N M R}(125 \mathrm{MHz}): \delta$ 172.3, 156.7, 156.1, 154.2, 151.6, 151.0, 149.8, 136.8, 129.5, 129.0, 128.8, 128.7, 128.3, 127.8, 114.9, 90.4, $85.4,84.1,83.7,82.4,79.2,69.0,66.9,54.7,54.3^{*}, 35.3,31.9,28.3,27.6,27.23^{*}, 27.17,25.3 ; 2$ resolved peaks arising specifically from the L-diastereomer $\mathbf{S 2 0 a}$ above are indicated with a *. ESI-MS $m / z$ (rel int): pos $904.3\left([\mathrm{M}+\mathrm{H}]^{+}, 45\right), 926.5\left([\mathrm{M}+\mathrm{Na}]^{+}, 100\right)$; neg $902.5\left([\mathrm{M}-\mathrm{H}]^{-}, 100\right)$, $938.8\left([\mathrm{M}+\mathrm{Cl}]^{-}, 30\right)$.

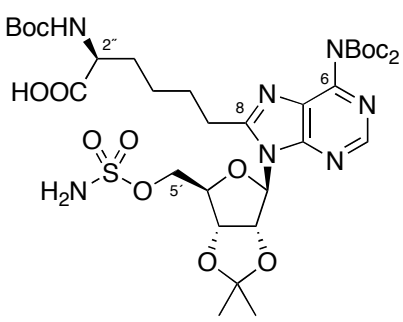

6- $N$-Bis $(t$-butoxycarbonyl)-8-( $N$-[t-butoxycarbonyl]-L-2"'butylglycin-6"'-yl)-2',3'-O-isopropylidene-5'-O-sulfamoyladenosine (S21a). Prepared by hydrogenation of alkene S21a (78 mg, $86 \mu \mathrm{mol}$, corrected for 2.5 equiv contaminating DMA) as described for S12a above with the following modification: Filtration of the reaction mixture through celite afforded 8-alkyladenosine carboxylic acid S21a (53 mg, 75\%) as a white solid that was used without further purification.

TLC: $R_{f} 0.12\left(9: 1 \mathrm{CH}_{2} \mathrm{Cl}_{2} / \mathrm{MeOH}\right)$. IR (NaCl, film): 3264 (N-H st), 2980, 1789 (C=O st), 1714 (C=O st), 1606, 1512, 1455, $1370\left(\mathrm{SO}_{2}\right.$ asym st), 1253, $1160\left(\mathrm{SO}_{2}\right.$ sym st), 1108, 999, 852. ${ }^{1} \mathrm{H}-\mathrm{NMR}\left(500 \mathrm{MHz}, \mathrm{CDCl}_{3}\right): \delta 8.82(\mathrm{~s}, 1 \mathrm{H}), 6.16(\mathrm{~s}, 1 \mathrm{H}), 5.90(\mathrm{~d}, 1 \mathrm{H}, J=5.5), 5.21(\mathrm{~m}, 2 \mathrm{H})$, $4.51(\mathrm{~m}, 1 \mathrm{H}), 4.23(\mathrm{~m}, 2 \mathrm{H}), 4.11(\mathrm{~m}, 1 \mathrm{H}), 3.04(\mathrm{~m}, 2 \mathrm{H}), 2.03-1.79(\mathrm{~m}, 3 \mathrm{H}), 1.72(\mathrm{~m}, 1 \mathrm{H}), 1.62$ $(\mathrm{m}, 4 \mathrm{H}), 1.47-1.20(\mathrm{~m}, 33 \mathrm{H}) ; 1$ exchangeable $\mathrm{H}$ not observed. ${ }^{13} \mathbf{C}-\mathbf{N M R}\left(125 \mathrm{MHz}, \mathrm{CDCl}_{3}\right)$ : $\delta$ 174.9, 158.3, 155.7, 153.8, 151.5, 150.8, 148.9, 128.3, 114.6, 89.9, 85.2, 84.3, 83.1, 81.5, 80.3, $68.9,60.4,34.7,31.9,28.3,27.9,27.0,26.4,25.3,24.1$. ESI-MS $\mathrm{m} / \mathrm{z}$ (rel int): pos 816.6 $\left([\mathrm{M}+\mathrm{H}]^{+}, 25\right), 838.4\left([\mathrm{M}+\mathrm{Na}]^{+}, 100\right)$; neg $814.8\left([\mathrm{M}-\mathrm{H}]^{-}, 100\right), 850.6\left([\mathrm{M}+\mathrm{Cl}]^{-}, 2\right)$.

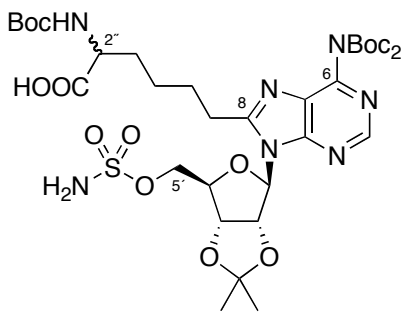

6- $N$-Bis(t-butoxycarbonyl)-8-( $N$-[ $t$-butoxycarbonyl]-DL-2"'butylglycin-6"-yl)-2',3'-O-isopropylidene-5'-O $\boldsymbol{O}$-sulfamoyladenosine $(\mathbf{S 2 1 b})$. Prepared from $\mathbf{S 2 0 b}$ as above. White powder (234 mg, 96\%). TLC: $R_{f} 0.12\left(9: 1 \mathrm{CH}_{2} \mathrm{Cl}_{2} / \mathrm{MeOH}\right) . \quad$ IR ( $\mathrm{NaCl}$, film): 3354 (N-H st), 2980, 1789 $(\mathrm{C}=\mathrm{O}$ st $), 1713$ (C=O st), 1607, 1514, 1454, $1370\left(\mathrm{SO}_{2}\right.$ asym st), 1253, $1160\left(\mathrm{SO}_{2} \mathrm{sym}\right.$ st $), 1108$, 1000, 852. ${ }^{1} \mathbf{H}-\mathbf{N M R}\left(400 \mathrm{MHz}, \mathrm{CDCl}_{3}\right): \delta 8.81(\mathrm{~s}, 1 \mathrm{H}), 6.15(\mathrm{~d}, 1 \mathrm{H}, J=3.8), 5.91(\mathrm{~m}, 1 \mathrm{H}), 5.22$ $(\mathrm{m}, 2 \mathrm{H}), 4.51(\mathrm{~m}, 1 \mathrm{H}), 4.24(\mathrm{~m}, 2 \mathrm{H}), 4.11(\mathrm{~m}, 1 \mathrm{H}), 3.01(\mathrm{~m}, 2 \mathrm{H}), 2.0-1.79(\mathrm{~m}, 3 \mathrm{H}), 1.78-1.42$ 
(m, 38H); 1 exchangeable $\mathrm{H}$ not observed. ${ }^{13} \mathbf{C}-\mathbf{N M R}\left(100 \mathrm{MHz}, \mathrm{CDCl}_{3}\right): \delta$ 174.7, 158.3, 155.7, $153.8 *, 153.7,151.4,150.7,148.9,128.3,114.6,89.9,85.1 *, 85.0,84.3,83.1,81.5,80.2 *, 80.1$, $68.9,58.4,35.4,32.0,28.4,27.9,27.0,26.6,26.4^{*}, 25.3,24.4,24.2^{*} ; 5$ resolved peak arising specifically from the L-diastereomer S21a above are indicated with a *. ESI-MS $m / z$ (rel int): pos $816.6\left([\mathrm{M}+\mathrm{H}]^{+}, 15\right), 838.4\left([\mathrm{M}+\mathrm{Na}]^{+}, 100\right)$; neg $814.9\left([\mathrm{M}-\mathrm{H}]^{-}, 100\right)$.

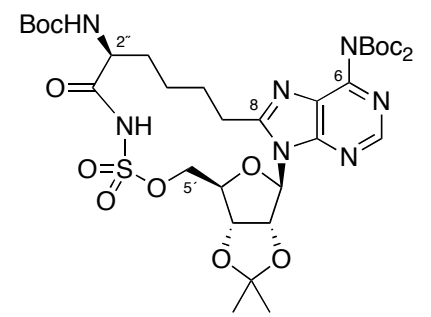

cyclo $^{8 \mathrm{C}_{3} \beta}$-6- $N$-Bis $(t$-butoxycarbonyl)-5' $-O$-( $N$-[ $N$-t-butoxycarbonyl-L-alanyl] sulfamoyl)adenosine (S22a). Prepared by macrocyclization of carboxylic acid S21a $(28.5 \mathrm{mg}, 35 \mu \mathrm{mol})$ as described for S13a above. Purification by silica flash chromatography (30:1 $\rightarrow$ 20:1 $\mathrm{CH}_{2} \mathrm{Cl}_{2} / \mathrm{MeOH}$ ) yielded macrocycle S22a $(17 \mathrm{mg}, 62 \%$, corrected for 2.5 equiv DBU contamination) as a white solid.

TLC: $R_{f} 0.34\left(9: 1 \mathrm{CH}_{2} \mathrm{Cl}_{2} / \mathrm{MeOH}\right)$. IR (NaCl, film): 3264 (N-H st), 2980, 1760 (C=O st), 1694 (C=O st), 1606, 1504, 1454, $1372\left(\mathrm{SO}_{2}\right.$ asym st), 1300, $1124\left(\mathrm{SO}_{2}\right.$ sym st), 849. ${ }^{1}$ H-NMR $\left(400 \mathrm{MHz}, \mathrm{CD}_{3} \mathrm{OD}\right): \delta 8.82(\mathrm{~s}, 1 \mathrm{H}), 6.46(\mathrm{~s}, 1 \mathrm{H}), 5.52(\mathrm{~m}, 1 \mathrm{H}), 5.13(\mathrm{~m}, 1 \mathrm{H}), 4.55(\mathrm{~m}, 2 \mathrm{H}), 4.45$ (m, 1H), $3.97(\mathrm{~m}, 1 \mathrm{H}), 3.35-3.14(\mathrm{~m}, 2 \mathrm{H}), 2.19(\mathrm{~m}, 1 \mathrm{H}), 2.04(\mathrm{~m}, 1 \mathrm{H}), 1.90(\mathrm{~m}, 2 \mathrm{H}), 1.55(\mathrm{~m}$, 35H); 2 exchangeable $\mathrm{H}$ not observed. ${ }^{13} \mathbf{C}-\mathbf{N M R}\left(100 \mathrm{MHz}, \mathrm{CD}_{3} \mathrm{OD}\right): \delta 181.7,161.2,157.3$ (2C), 155.5, 152.4, 151.6, 149.6, 129.5, 116.6, 90.2, 85.4, 84.7, 81.9, 80.5, 69.5, 57.4, 33.8, 30.0, 29.2, 28.4, 28.0, 24.9, 24.7, 20.4. ESI-MS $m / z$ (rel int): pos $798.4\left([\mathrm{M}+\mathrm{H}]^{+}, 100\right), 820.4$ $\left([\mathrm{M}+\mathrm{Na}]^{+}, 40\right) ;$ neg $796.6\left([\mathrm{M}-\mathrm{H}]^{-}, 100\right)$.

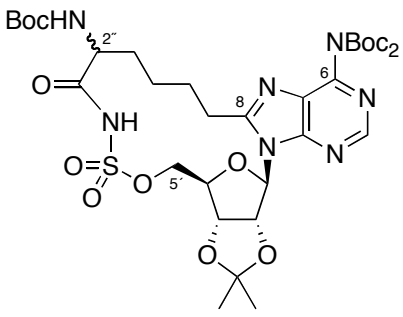

cyclo $^{8 \mathrm{C}_{3} \beta}$-6- $N$-Bis(t-butoxycarbonyl)-5'-O-( $N$-[N-t-butoxycarbonyl-DL-alanyl] sulfamoyl)adenosine (S22b). Prepared by macrocyclization of carboxylic acid S21b (234 mg, $0.287 \mathrm{mmol})$ as described for S13a above. The reaction was quenched by addition of satd aq $\mathrm{NH}_{4} \mathrm{Cl}(25 \mathrm{~mL})$ and extracted with EtOAc $(3 \times 50 \mathrm{~mL})$. The combined organic extracts were washed with $\mathrm{NH}_{4} \mathrm{Cl}(2 \times 20 \mathrm{~mL})$ and $\mathrm{NaCl}(1 \times 20 \mathrm{~mL})$, dried $\left(\mathrm{Mg}_{2} \mathrm{SO}_{4}\right)$, filtered, and concentrated by rotary evaporation. Purification by silica flash chromatography (1:9 hexanes/EtOAc) yielded $\mathbf{S 2 2 b}$ as a white powder (66 mg, 28\%). TLC: $R_{f} 0.34\left(9: 1 \mathrm{CH}_{2} \mathrm{Cl}_{2} / \mathrm{MeOH}\right)$. IR $(\mathrm{NaCl}$, film): $3264(\mathrm{~N}-\mathrm{H}$ st), 2980, 1760 (C=O st), 1694 (C=O st), 1606, 1504, 1454, 1372 ( $\mathrm{SO}_{2}$ asym st), 1300, $1124\left(\mathrm{SO}_{2}\right.$ sym st), 849. ${ }^{1}$ H-NMR (400 MHz, $\left.\mathrm{CD}_{3} \mathrm{OD}\right): \delta 8.78(\mathrm{~s}, 1 \mathrm{H}), 6.45(\mathrm{~m}, 1 \mathrm{H}), 5.52(\mathrm{~m}, 1 \mathrm{H}), 5.17$ 
$(\mathrm{m}, 1 \mathrm{H}), 4.39(\mathrm{~m}, 2 \mathrm{H}), 4.26(\mathrm{~m}, 1 \mathrm{H}), 4.01(\mathrm{~m}, 0.5 \mathrm{H}$, L-stereoisomer $), 3.95(\mathrm{~m}, 0.5 \mathrm{H}$, D-stereoisomer), 3.35-3.14 (m, 2H), $2.19(\mathrm{~m}, 1 \mathrm{H}), 2.04(\mathrm{~m}, 1 \mathrm{H}), 1.90(\mathrm{~m}, 2 \mathrm{H}), 1.55(\mathrm{~m}, 35 \mathrm{H}) ; 2$ exchangeable $\mathrm{H}$ not observed. ${ }^{13} \mathrm{C}$-NMR (100 MHz, $\left.\mathrm{CD}_{3} \mathrm{OD}\right): \delta 180.7,161.2^{*}, 161.1,157.3$ (2C), 155.6, 152.4, 151.59*, 151.55, 149.5, 129.6*, 129.3, 117.0, 116.8*, 89.9*, 89.7, 85.40*, 85.36, 84.6*, 84.4, 81.6*, 81.5, 80.4, 69.5, 57.9, 57.4*, 33.8*, 33.6, 29.9, 29.2, 28.2, 28.0, 25.1, 24.7, 21.3; 10 resolved peaks arising specifically from the L-diastereomer S22a above are indicated with a *. ESI-MS $m / z$ (rel int): $\operatorname{pos} 798.4\left([\mathrm{M}+\mathrm{H}]^{+}, 10\right), 820.4\left([\mathrm{M}+\mathrm{Na}]^{+}, 100\right)$; neg $796.6\left([\mathrm{M}-\mathrm{H}]^{-}, 100\right)$.

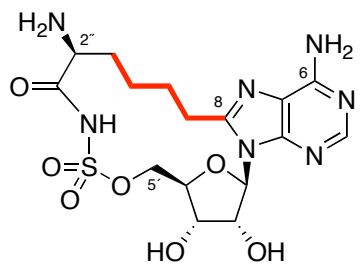

cyclo $^{8 \mathrm{C}_{3} \beta}-5^{\prime}-O-\left(N\right.$-[L-alanyl]sulfamoyl)adenosine $\left(2 \mathrm{~b}\right.$, cyclo $\left.^{8 \mathrm{C}_{3} \beta}-\mathrm{L}-\mathrm{Ala}-\mathrm{AMS}\right)$. Prepared by TFA deprotection of macrocycle S22a $(2.0 \mathrm{mg}, 2.5 \mu \mathrm{mol}$, corrected for 2.5 equiv contaminating $\mathrm{DBU})$ as described for 2a above. Purification by preparative $\mathrm{HPLC}\left(\mathrm{C}_{18} ; 150\right.$ x $21.4 \mathrm{~mm}$; gradient $0-20 \% \mathrm{CH}_{3} \mathrm{CN}$ in $\mathrm{H}_{2} \mathrm{O}$ with $0.05 \%$ TFA over $20 \mathrm{~min} ; 16 \mathrm{~mL} / \mathrm{min}$ ) yielded macrocycle 2b $(1.1 \mathrm{mg}, 96 \%)$ as a white powder.

HPLC: $t_{\text {ret }}=15.5$ min. IR ( NaCl, film): $3142(\mathrm{~N}-\mathrm{H}, \mathrm{O}-\mathrm{H}$ st $), 2954,1685$ (C=O st), 1619, 1437, 1296 ( $\mathrm{SO}_{2}$ asym st), 1202, 1130 ( $\mathrm{SO}_{2}$ sym st), 832. ${ }^{1} \mathbf{H}-\mathbf{N M R}\left(500 \mathrm{MHz}, \mathrm{CD}_{3} \mathrm{OD}\right.$; peak assignments based on COSY analysis): $\delta 8.30(\mathrm{~s}, 1 \mathrm{H}, \mathrm{C} 2-\mathrm{H}), 6.06\left(\mathrm{~d}, 1 \mathrm{H}, J=3.9, \mathrm{C}^{\prime}{ }^{\prime}-\mathrm{H}\right), 5.00$ $\left(\mathrm{dd}, 1 \mathrm{H}, J=3.9,7.7, \mathrm{C}^{\prime}-\mathrm{H}\right), 4.64\left(\mathrm{~d}, 1 \mathrm{H}, J=6.1,6.1, \mathrm{C}^{\prime}-\mathrm{H}\right), 4.49(\mathrm{dd}, 1 \mathrm{H}, J=11.3,1.8$, $\left.\mathrm{C}^{\prime}-\mathrm{H}_{\mathrm{a}}\right), 4.30\left(\mathrm{dd}, 1 \mathrm{H}, J=5.1,11.4, \mathrm{C}^{\prime}-\mathrm{H}\right), 4.22\left(\mathrm{~m}, 1 \mathrm{H}, \mathrm{C}^{\prime}-\mathrm{H}_{\mathrm{b}}\right), 3.73(\mathrm{dd}, 1 \mathrm{H}, \mathrm{J}=3.9,6.8$,

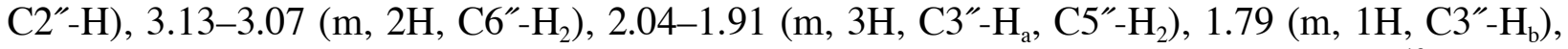
$1.53\left(\mathrm{~m}, 1 \mathrm{H}, \mathrm{C} 4 "-\mathrm{H}_{\mathrm{a}}\right), 1.39\left(\mathrm{~m}, 1 \mathrm{H}, \mathrm{C}^{\prime \prime}-\mathrm{H}_{\mathrm{b}}\right) ; 7$ exchangeable $\mathrm{H}$ not observed. ${ }^{13} \mathbf{C}-\mathbf{N M R}$ (125 MHz, $\left.\mathrm{CD}_{3} \mathrm{OD}\right): \delta 173.3,157.8,151.8,151.0,146.0,119.0,90.9,83.1,73.7,70.9,70.3$, 55.9, 31.0, 28.4, 27.3, 23.1. ESI-MS $m / z$ (rel int): pos 458.1 ([M+H] $\left.]^{+}, 100\right), 480.1\left([\mathrm{M}+\mathrm{Na}]^{+}\right.$, $35)$; neg $456.4\left([\mathrm{M}-\mathrm{H}]^{-}, 100\right)$.

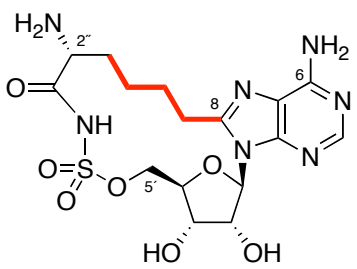

cyclo $^{8 \mathrm{C}_{3} \beta}-5^{\prime}-O-\left(N\right.$-[L-alanyl]sulfamoyl)adenosine (2d, cyclo $\left.^{8 \mathrm{C}_{3} \beta}-\mathrm{D}-\mathrm{Ala}-\mathrm{AMS}\right)$. Prepared from S22b as above with isolation of the D-diastereomer by preparative HPLC $\left(\mathrm{C}_{18} ; 150 \times 21.4 \mathrm{~mm}\right.$; gradient $0-20 \% \mathrm{CH}_{3} \mathrm{CN}$ in $\mathrm{H}_{2} \mathrm{O}$ with $0.05 \%$ TFA over $20 \mathrm{~min} ; 16 \mathrm{~mL} / \mathrm{min}$ ). White powder (5.9 mg, $26 \%$, versus $50 \%$ theoretical maximum). HPLC: $t_{\mathrm{ret}}=14.2 \mathrm{~min}$. IR $(\mathrm{NaCl}$, film): 3154 (N-H, O-H st), 2919, 1684 (C=O st), 1613, 1437, 1302 ( $\mathrm{SO}_{2}$ asym st), 1196, 1138 ( $\mathrm{SO}_{2}$ sym st), 838. ${ }^{1}$ H-NMR (500 MHz, $\mathrm{CD}_{3} \mathrm{OD}$ ): $\delta 8.29$ (s, 1H), 6.08 (d, 1H, J = 3.5), 4.93 (obsc, 1H), 4.68 
$(\mathrm{m}, 1 \mathrm{H}), 4.56(\mathrm{dd}, 1 \mathrm{H}, J=11.8,1.8), 4.35(\mathrm{dd}, 1 \mathrm{H}, J=11.8,4.8), 4.19(\mathrm{~m}, 1 \mathrm{H}), 3.63(\mathrm{dd}, 1 \mathrm{H}, J=$ 6.5, 4.5), $3.14(\mathrm{~m}, 1 \mathrm{H}), 3.03(\mathrm{~m}, 1 \mathrm{H}), 2.03-1.99(\mathrm{~m}, 2 \mathrm{H}), 1.81(\mathrm{~m}, 2 \mathrm{H}), 1.56(\mathrm{~m}, 1 \mathrm{H}), 1.43(\mathrm{~m}$, $1 \mathrm{H}) ; 7$ exchangeable $\mathrm{H}$ not observed. ${ }^{13} \mathrm{C}-\mathbf{N M R}\left(125 \mathrm{MHz}, \mathrm{CD}_{3} \mathrm{OD}\right): \delta 173.5,157.8,151.7$, 151.0, 145.8, 119.0, 90.6, 83.2, 73.7, 70.8, 70.4, 56.0, 31.3, 28.2, 27.1, 23.6. ESI-MS m/z (rel int): $\operatorname{pos} 458.2\left([\mathrm{M}+\mathrm{H}]^{+}, 100\right), 480.2\left([\mathrm{M}+\mathrm{Na}]^{+}, 30\right) ; \operatorname{neg} 456.4\left([\mathrm{M}-\mathrm{H}]^{-}, 100\right)$.

\section{G. SYNTHESIS OF CYCLO ${ }^{8 \mathrm{C}_{3} \beta}$-PROPIONYL-AMS MACROCYCLE $(2 \mathrm{e})$}

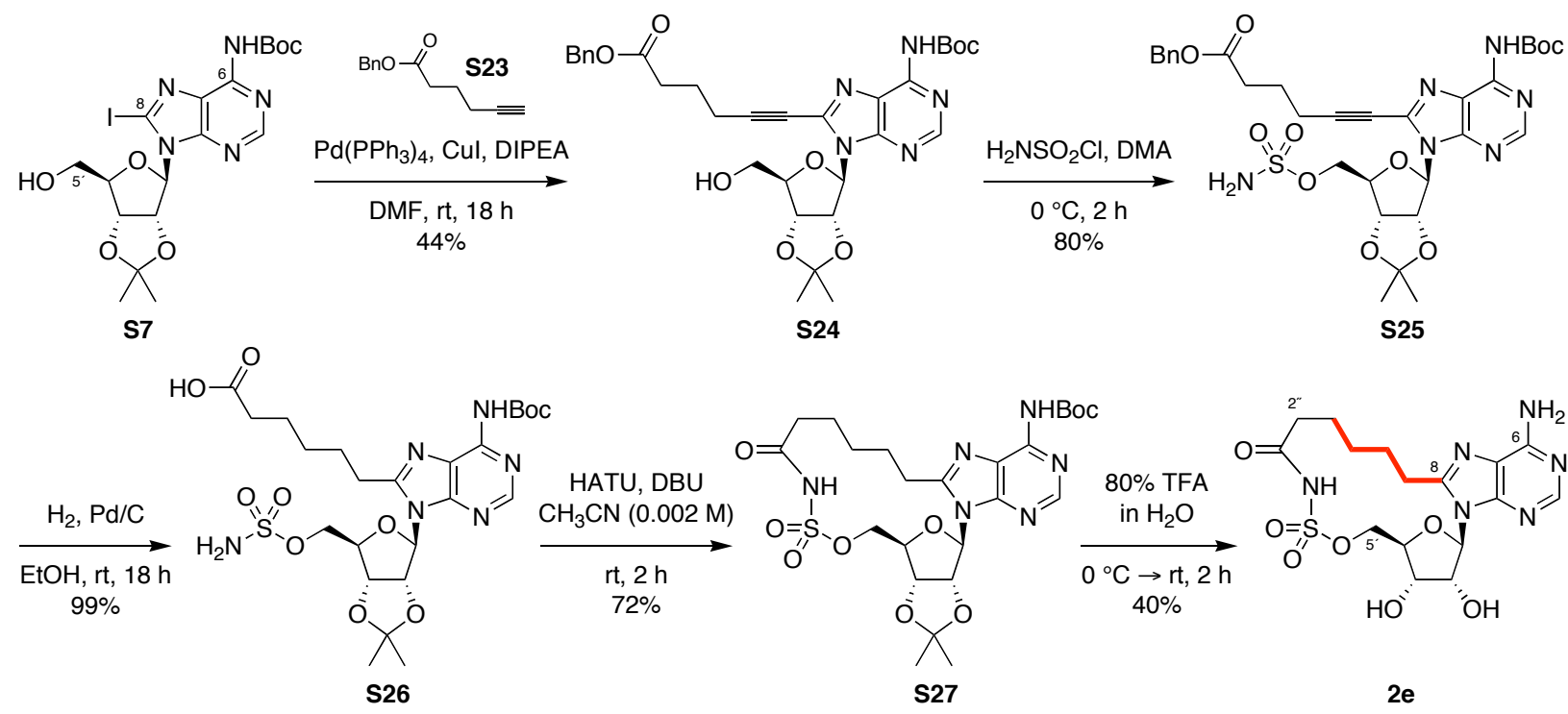

Figure S6. Synthesis of desamino macrocycle $2 \mathrm{e}$ having a 3-carbon linker (red). Macrocycle $2 \mathrm{e}$ was synthesized by a Sonogashira cross coupling-based route similar to that used to synthesize 2 a and 2c.

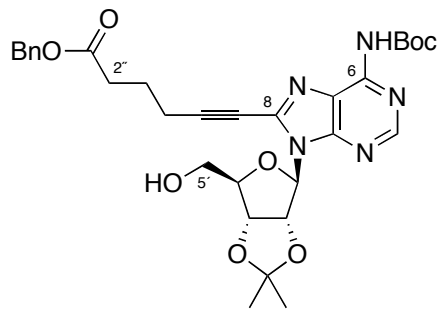

6- $N$-(t-Butoxycarbonyl)-8-(5-[carbobenzyloxy]-1-pentyn-1-yl)-2',3'-O-isopropylideneadenosine (S24). Prepared by Sonogashira cross coupling of 8-iodoadenosine S7 (240 mg, $0.450 \mathrm{mmol}, 1.0$ equiv) and benzyl 5-hexynoate $\mathbf{S 2 3}$ (150 mg, $0.742 \mathrm{mmol}, 1.6$ equiv) as described for S9a above. Purification by silica flash chromatography (3:2 hexanes/EtOAc) afforded 8-alkynyladenosine S24 (119 $\mathrm{mg}, 44 \%$ ) as a white solid.

TLC: $R_{f} 0.19$ (1:1 hexanes/EtOAc). IR (NaCl, film): $3311(\mathrm{~N}-\mathrm{H}$ st, O-H st), 2982, $1756(\mathrm{C}=\mathrm{O}$ st), 1616, 1524, 1483, 1435, 1354, 1286, 1234, 1209, 1154, 1112, 1027, 874. ${ }^{\mathbf{1}} \mathbf{H}-\mathbf{N M R}$ $\left(500 \mathrm{MHz}, \mathrm{CDCl}_{3}\right): \delta 8.71(\mathrm{~s}, 1 \mathrm{H}, \mathrm{C} 2-\mathrm{H}), 8.02(\mathrm{~s}, 1 \mathrm{H}, \mathrm{N} 6-\mathrm{H}), 7.34(\mathrm{~m}, 5 \mathrm{H}, \mathrm{Ar}), 6.24(\mathrm{~d}, 1 \mathrm{H}, J=$ 
5.2, $\left.\mathrm{C} 1^{\prime}-\mathrm{H}\right), 5.98\left(\mathrm{~d}, 1 \mathrm{H}, J=5.5, \mathrm{C}^{\prime}-\mathrm{OH}\right), 5.21\left(\mathrm{t}, 1 \mathrm{H}, J=6.5, \mathrm{C} 2^{\prime}-\mathrm{H}\right), 5.14\left(\mathrm{~s}, 2 \mathrm{H}, \mathrm{Bn}-\mathrm{CH}_{2}\right)$, $5.09\left(\mathrm{~d}, 1 \mathrm{H}, J=5.1, \mathrm{C} 3^{\prime}-\mathrm{H}\right), 4.54\left(\mathrm{~s}, 1 \mathrm{H}, \mathrm{C}^{\prime}-\mathrm{H}\right), 3.98\left(\mathrm{~d}, 1 \mathrm{H}, J=11.3, \mathrm{C}^{\prime}-\mathrm{H}_{\mathrm{a}}\right), 3.79(\mathrm{t}, 1 \mathrm{H}, J=$ 11.6, C5'- $\mathrm{H}_{\mathrm{b}}$ ), 2.64 (t, 2H, $\left.J=7.1, \mathrm{C}^{\prime \prime}-\mathrm{H}_{2}\right), 2.57$ (t, 2H, $\left.J=7.3, \mathrm{C} 4^{\prime \prime}-\mathrm{H}_{2}\right), 2.03(\mathrm{t}, 2 \mathrm{H}, J=7.2$, $\left.\mathrm{C}^{\prime \prime}-\mathrm{H}_{2}\right), 1.63\left(\mathrm{~s}, 3 \mathrm{H}\right.$, acetonide- $\left.\mathrm{CH}_{3}\right), 1.55(\mathrm{~s}, 9 \mathrm{H}, t-\mathrm{Bu}), 1.37\left(\mathrm{~s}, 3 \mathrm{H}\right.$, acetonide- $\left.\mathrm{CH}_{3}\right) .{ }^{13} \mathbf{C}-\mathbf{N M R}$ $\left(125 \mathrm{MHz}, \mathrm{CDCl}_{3}\right): \delta 172.3,153.2,150.1,149.1,149.0,136.5,135.7,133.8,133.6,132.1,122.0$, 114.0, 98.8, 93.1, 85.8, 82.8, 82.6, 81.7, 69.8, 66.5, 63.3, 32.9, 28.0, 27.7, 25.4, 23.0, 18.9 . ESI-MS $m / z$ (rel int): pos $608.0\left([\mathrm{M}+\mathrm{H}]^{+}, 90\right), 629.9\left([\mathrm{M}+\mathrm{Na}]^{+}, 100\right)$; neg $606.0\left([\mathrm{M}-\mathrm{H}]^{-}, 100\right)$, $642.1\left([\mathrm{M}+\mathrm{Cl}]^{-}, 11\right)$.

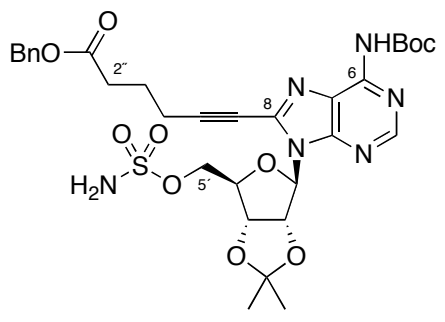

6- $N$-(t-Butoxycarbonyl)-8-(5-[carbobenzyloxy]-1-pentyn-1-yl)-2',3'-O-isopropylidene-5' $-O$ sulfamoyladenosine (S25). Prepared by sulfamoylation of alcohol S24 (110 mg, $0.181 \mathrm{mmol})$ as described for S11a above. Purification by silica flash chromatography $\left(30: 1 \mathrm{CH}_{2} \mathrm{Cl}_{2} / \mathrm{MeOH}\right)$ afforded 5'-O-sulfamoyladenosine $\mathbf{S 2 5}$ (100 mg, 80\%) as a white solid.

TLC: $R_{f} 0.19$ (1:1 hexanes/EtOAc). IR (NaCl, film): 3237 (N-H st), 2982, 2242, 1735 (C=O st), 1702 (C=O st), 1607, 1516, 1480, 1457, 1428, 1371 ( $\mathrm{SO}_{2}$ asym st), 1331, 1233, $1144\left(\mathrm{SO}_{2}\right.$ sym st), 1103, 999, 867. ${ }^{1} \mathbf{H}-\mathbf{N M R}\left(500 \mathrm{MHz}\right.$, acetone- $\left.d_{6}\right): \delta 8.94(\mathrm{~s}, 1 \mathrm{H}), 8.59(\mathrm{~s}, 1 \mathrm{H}), 7.36(\mathrm{~m}, 5 \mathrm{H})$, $6.73(\mathrm{~s}, 2 \mathrm{H}), 6.38(\mathrm{~d}, 1 \mathrm{H}, J=3.0), 5.69(\mathrm{dd}, 1 \mathrm{H}, J=6.3,1.8), 5.29(\mathrm{dd}, 1 \mathrm{H}, J=6.2,3.4), 5.16(\mathrm{~s}$, 2H), 4.47 (dt, $1 \mathrm{H}, J=6.3,3.4), 4.39$ (dd, 1H, $J=10.4,6.0), 4.24$ (dd, 1H, $J=10.4,6.9), 2.76$ (t, $2 \mathrm{H}, J=7.1), 2.64(\mathrm{t}, 2 \mathrm{H}, J=7.4), 2.05$ (obsc, $2 \mathrm{H}), 1.53(\mathrm{~m}, 12 \mathrm{H}), 1.37(\mathrm{~s}, 3 \mathrm{H}) .{ }^{13} \mathbf{C}-\mathbf{N M R}$ $\left(125 \mathrm{MHz}\right.$, acetone- $\left.d_{6}\right): \delta 170.5,151.3,151.2,148.7,148.6,148.4,135.0,134.8,132.5,131.7$, 120.0, 112.5, 97.0, 88.4, 84.0, 81.8, 80.6, 79.1, 68.7, 66.9, 64.2, 31.0, 29.9, 26.3, 26.0, 21.7 16.7. ESI-MS $m / z$ (rel int): pos $687.0\left([\mathrm{M}+\mathrm{H}]^{+}, 50\right), 708.9\left([\mathrm{M}+\mathrm{Na}]^{+}, 100\right)$; neg $685.0\left([\mathrm{M}-\mathrm{H}]^{-}, 100\right)$, $721.0\left([\mathrm{M}+\mathrm{Cl}]^{-}, 30\right)$.

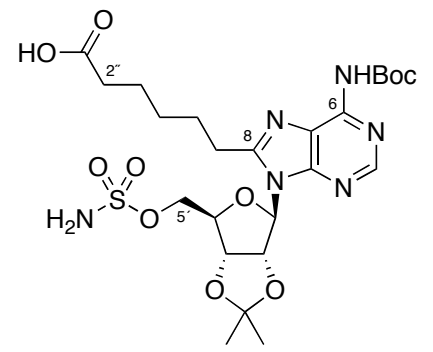

6- $\mathrm{N}$-( $t$-Butoxycarbonyl)-8-(5-carboxypent-1-yl)-2', $3^{\prime}$ - $O$-isopropylidene-5' $O$-sulfamoyladenosine (S26). Prepared by hydrogenation of 8-alkynyladenosine S25 (90 mg, $0.131 \mathrm{mmol}$ ) as described for S12a above. Purification by silica flash chromatography (EtOAc, $0.5 \% \mathrm{AcOH})$ afforded 8-alkyladenosine carboxylic acid S26 (78 $\mathrm{mg}, 99 \%)$ as a white solid. 
TLC: $R_{f} 0.34\left(9: 1 \mathrm{CH}_{2} \mathrm{Cl}_{2}\right)$. IR (NaCl, film): 3311 (N-H st, O-H st), 2943, 2831, 1752 (C=O st), 1651 (C=O st), 1616, 1531, 1449, 1410 ( $\mathrm{SO}_{2}$ asym st), 1270, 1165 ( $\mathrm{SO}_{2}$ sym st), 1112, 1023, 864. ${ }^{1}$ H-NMR $\left(500 \mathrm{MHz}, \mathrm{MeOD}-d_{4}\right): \delta 8.44(\mathrm{~s}, 1 \mathrm{H}), 6.1(\mathrm{~d}, 1 \mathrm{H}, J=7.4), 5.67(\mathrm{~d}, 1 \mathrm{H}, J=3.8)$, $5.16(\mathrm{dd}, 1 \mathrm{H}, J=6.1,3.2), 4.35(\mathrm{~m}, 1 \mathrm{H}), 4.17(\mathrm{dd}, 1 \mathrm{H}, J=10.5,5.4), 4.03(\mathrm{dd}, 1 \mathrm{H}, J=10.5$, 6.8), $2.95(\mathrm{~m}, 2 \mathrm{H}), 2.25(\mathrm{~m}, 2 \mathrm{H}), 1.79(\mathrm{~m}, 2 \mathrm{H}), 1.49(\mathrm{~m}, 16 \mathrm{H}), 1.29(\mathrm{~s}, 3 \mathrm{H}) ; 4$ exchangable protons not observed. ${ }^{13} \mathrm{C}-\mathrm{NMR}\left(125 \mathrm{MHz}, \mathrm{MeOD}-d_{4}\right): \delta 175.1,158.8,153.0,152.9,150.1$, 122.0, 115.6, 91.3, 86.5, 84.8, 83.2, 82.9, 69.6, 34.8, 34.7, 29.6, 28.5, 28.5 28.0, 27.4, 25.7, 25.5. ESI-MS $m / z$ (rel int): pos $600.9\left([\mathrm{M}+\mathrm{H}]^{+}, 100\right), 623.0\left([\mathrm{M}+\mathrm{Na}]^{+}, 20\right)$; neg $599.0\left([\mathrm{M}-\mathrm{H}]^{-}, 100\right)$, $634.8\left([\mathrm{M}+\mathrm{Cl}]^{-}, 8\right)$.

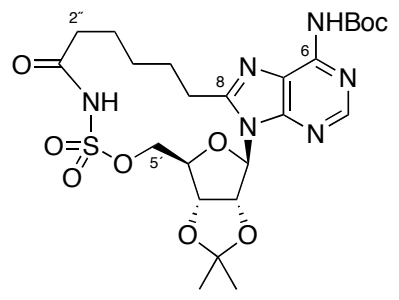

cyclo $^{8 \mathrm{C}_{3} \beta}-6-\mathrm{N}$-( $t$-Butoxycarbonyl)-5' $-O$-( $N$-propionylsulfamoyl)adenosine (S27). Prepared by macrocyclization of carboxylic acid S26 (43 mg, $72 \mu \mathrm{mol}$ ) as described for S13a above. Purification by silica flash chromatography $\left(20: 1 \quad \mathrm{CH}_{2} \mathrm{Cl}_{2} / \mathrm{MeOH}\right)$ afforded macrocycle S27 (30 $\mathrm{mg} 72 \%$ ) as a white solid.

TLC: $R_{f} 0.47\left(9: 1 \mathrm{CH}_{2} \mathrm{Cl}_{2}\right)$. IR (NaCl, film): 3300 (N-H st), 2845, 1705 (C=O st), 1578, 1560, 1358 ( $\mathrm{SO}_{2}$ asym st), 1323, 1248, 1124 ( $\mathrm{SO}_{2}$ sym st), 1089. ${ }^{1} \mathbf{H}-\mathbf{N M R}\left(500 \mathrm{MHz}, \mathrm{CD}_{3} \mathrm{OD}\right): \delta 8.44$ $(\mathrm{s}, 1 \mathrm{H}), 6.16(\mathrm{~s}, 1 \mathrm{H}), 5.61(\mathrm{dd}, 1 \mathrm{H}, J=6.2,1.3), 5.17(\mathrm{dd}, 1 \mathrm{H}, J=6.0,4.0), 4.46(\mathrm{~m}, 2 \mathrm{H}), 4.18$ $(\mathrm{m}, 1 \mathrm{H}), 2.89(\mathrm{~m}, 2 \mathrm{H}), 2.24(\mathrm{~m}, 1 \mathrm{H}), 2.07(\mathrm{~m}, 1 \mathrm{H}), 1.84(\mathrm{~m}, 1 \mathrm{H}), 1.73(\mathrm{~m}, 1 \mathrm{H}), 1.58-1.35(\mathrm{~m}$, 16H), 1.29 (s, 3H); 2 exchangable $\mathrm{H}$ not observed. ${ }^{13} \mathbf{C}$-NMR (125 MHz, MeOD- $\left.d_{4}\right): \delta 174.2$, $157.9,152.9,152.6,152.4,150.3,122.2,115.8,90.7,86.6,85.0,82.8,82.3,73.9,37.6,35.2$, 28.4, 27.6, 27.4, 25.6, 25.5, 24.0. ESI-MS $m / z$ (rel int): pos 582.9 ([M+H] $\left.]^{+}, 100\right), 605.0$ $\left([\mathrm{M}+\mathrm{Na}]^{+}, 90\right)$; neg $581.0\left([\mathrm{M}-\mathrm{H}]^{-}\right)$.

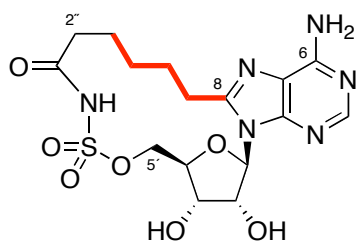

cyclo $^{8 \mathrm{C}_{3} \beta}-5^{\prime}-O$-( $N$-propionylsulfamoyl)adenosine (2e, cyclo $^{8 \mathrm{C}_{3} \beta}$-propionyl-AMS). Prepared by TFA deprotection of macrocycle S27 as described for 2a above. Purification by preparative HPLC $\left(\mathrm{C}_{18} ; 250 \times 21.4 \mathrm{~mm}\right.$; gradient $0-40 \% \mathrm{CH}_{3} \mathrm{CN}$ in $\mathrm{H}_{2} \mathrm{O}$ with $0.05 \%$ TFA over 20 min; $16 \mathrm{~mL} / \mathrm{min})$ yielded macrocycle $2 \mathbf{e}(3.0 \mathrm{mg}, 40 \%)$ as a white solid.

HPLC: $t_{\text {ret }}=13.6$ min. IR (NaCl, film): 3128 (N-H st), 2940, 1680 (C=O st), 1521, 1446, 1381, 1313 ( $\mathrm{SO}_{2}$ asym st), 1182, 1135 ( $\mathrm{SO}_{2}$ sym st), 1024, 969, 839. ${ }^{1} \mathbf{H}-\mathbf{N M R}(500 \mathrm{MHz}): \delta 8.09$ (s, $1 \mathrm{H}), 5.96(\mathrm{~d}, 1 \mathrm{H}, J=4.8), 4.88(\mathrm{dd}, 1 \mathrm{H}, J=6.2,4.8), 4.43(\mathrm{t}, 1 \mathrm{H}, J=6.0), 4.31(\mathrm{~m}, 1 \mathrm{H}), 4.17$ 
$(\mathrm{m}, 2 \mathrm{H}), 2.97(\mathrm{~m}, 2 \mathrm{H}), 2.16(\mathrm{~m}, 2 \mathrm{H}), 1.94(\mathrm{~m}, 1 \mathrm{H}), 1.77(\mathrm{~m}, 1 \mathrm{H}), 1.57(\mathrm{~m}, 2 \mathrm{H}), 1.38(\mathrm{~m}, 2 \mathrm{H})$. ${ }^{13}$ C-NMR (125 MHz): $\delta 172.3,157.4,153.3,151.0,148.0,119.4,91.6,81.5,73.8,73.2,71.4$, 35.0, 28.6, 27.6, 24.2, 23.8. ESI-MS $m / z$ (rel int): pos $443.0\left([\mathrm{M}+\mathrm{H}]^{+}\right)$; neg $441.1\left([\mathrm{M}-\mathrm{H}]^{-}\right)$.

\section{H. HMWP2 INHIBITION ASSAY}

\section{Protein Expression and Purification}

HMWP2 ${ }^{1-1491}$ was expressed in E. coli BL21(DE3) (Invitrogen) as an isopropyl $\beta$-D-1-thiogalactopyranoside (IPTG)-inducible, C-terminally His $_{6}$-tagged protein using plasmid pET22b-irp2/1-1491. ${ }^{20} \quad$ For protein production, the strain E. coli BL21(DE3)/pET22b-irp2/1-1491, expressing HMWP2 ${ }^{1-1491}-\mathrm{His}_{6}$, was cultivated in LB broth ${ }^{21}$ (9 L) containing ampicillin $(100 \mu \mathrm{g} / \mathrm{mL})$ with orbital shaking $(220 \mathrm{rpm})$ at $30{ }^{\circ} \mathrm{C}$. When the culture reached $\mathrm{OD}_{600}=0.6$, the temperature was lowered to $25^{\circ} \mathrm{C}$. After a $1 \mathrm{~h}$ incubation period, the culture was induced with $1 \mathrm{mM}$ IPTG for $4 \mathrm{~h}$. Subsequently, the cells were harvested by centrifugation and resuspended in lysis buffer $(75 \mathrm{~mL}, 50 \mathrm{mM}$ Tris $\cdot \mathrm{HCl} \mathrm{pH} 8,0.5 \mathrm{M} \mathrm{NaCl}$, $20 \%$ sucrose, $1 \mathrm{mM} \beta$-mercaptoethanol, $10 \mathrm{mM}$ imidazole, $0.1 \%$ IGEPAL). Resuspended cells were disrupted using a French pressure cell and the cellular debris was removed from the lysates by ultracentrifugation. HMWP2 ${ }^{1-1491}-\mathrm{His}_{6}$ was purified from cell lysates by nickel column chromatography using Ni·NTA Superflow resin (Qiagen) according to the manufacturer's instructions. Fractions containing HMWP2 $2^{1-1491}-\mathrm{His}_{6}$ were pooled and concentrated ( 6-fold) using centrifugal filter devices (Millipore Centriprep YM-50, $50 \mathrm{kDa}$ cutoff). The concentrated sample was applied to a size exclusion column (XK26/100 packed with Superdex 200; Amersham Pharmacia Biosciences) previously equilibrated with running buffer (50 mM Tris $\mathrm{pH} 7.5,300 \mathrm{mM} \mathrm{NaCl}$ ) for further purification of $\mathrm{HMWP}^{1-1491}-\mathrm{His}_{6}$. Eluting fractions containing purified $\mathrm{HMWP} 2^{1-1491}-\mathrm{His}_{6}$ were pooled, concentrated $(\leq 10 \mathrm{mg} / \mathrm{ml})$, and stored at $-80{ }^{\circ} \mathrm{C}$.

\section{DETERMINATION OF $K_{\mathrm{i}}^{\text {app }}$ AND $K_{\mathrm{i}}$ VALUES}

Inhibition constants for HMWP2 $2^{1-1491}-\mathrm{His}_{6}$ adenylation activity were determined using an ATP$\left[{ }^{32} \mathrm{P}\right]$-pyrophosphate (PPi) exchange assay as previously described. ${ }^{22,23}$ All assays were performed in triplicate. Enzymatic reactions $(50 \mu \mathrm{L})$ containing $75 \mathrm{mM}$ Tris $\cdot \mathrm{HCl}(\mathrm{pH} 8.8)$ $10 \mathrm{mM} \mathrm{MgCl}_{2}, 1 \mathrm{mM}$ TCEP, $5 \%$ glycerol, $1 \mathrm{mM}$ sodium $\left[{ }^{32} \mathrm{P}\right]-\mathrm{PPi}$ (5 Ci/mol, PerkinElmer), and variable concentrations of ATP and L-cysteine were initiated by addition of HMWP2 ${ }_{1-1491}$ at $30 \mathrm{nM}$ final concentration. Inhibitors were added in DMSO (1.2\% of total volume). Reactions were incubated at $37^{\circ} \mathrm{C}$ for $15 \mathrm{~min}$. To determine $K_{\mathrm{i}}^{\text {app }}$ values, reactions were performed using

\footnotetext{
${ }^{20}$ Gehring, A. M.; Mori, I.; Perry, R. D.; Walsh, C. T. Biochemistry 1998, 37, 11637-11650.

${ }^{21}$ Sambrook, J.; Fritsch, E. F.; Maniatis, T. Molecular cloning. A laboratory manual.; 2nd ed.; Cold Spring Harbor Laboratory Press: New York, 1989.

${ }^{22}$ Quadri, L. E. N.; Keating, T. A.; Patel, H. M.; Walsh, C. T. Biochemistry 1999, 38, 14941-14954.

${ }^{23}$ Quadri, L. E. N.; Sello, J.; Keating, T. A.; Weinreb, P. H.; Walsh, C. T. Chem. Biol. 1998, 5, 631-645.
} 
ATP and L-cysteine at $3 \mathrm{mM}$. Dose-response data sets were fitted to the Morrison equation for tight binding inhibitors: ${ }^{24}$

$$
\frac{v_{i}}{v_{c}}=1-\frac{\left([E]+[I]+K_{i}^{a p p}\right)-\sqrt{\left([E]+[I]+K_{i}^{a p p}\right)^{2}-4[E][I]}}{2[E]}
$$

where $v_{i}$ and $v_{c}$ are the activities measured in inhibitor-containing reactions and DMSOcontaining $(1.2 \%)$ controls, respectively. To determine $K_{\mathrm{i}}$ values, two series of dose-response experiments were performed to obtain $K_{\mathrm{i}}^{\text {app }}$ values at different substrate concentrations (Figures S7, S8, S9). The plot of $K_{\mathrm{i}}^{\text {app }}$ values versus substrate concentrations were then fit to the equation for competitive tight binding inhibitors: ${ }^{24}$

$$
K_{i}^{a p p}=K_{i}\left(1+\frac{[S]}{K_{m}}\right)
$$

In the first series, the ATP concentration was fixed at $5 \mathrm{mM}$ (for experiments with L-Cys-AMS) or $3 \mathrm{mM}$ (for experiments with other inhibitors) and the L-cysteine concentration was varied between 0-5 mM (for experiments with L-Cys-AMS) or 0-3 $\mathrm{mM}$ (for experiments with other inhibitors). In the second series, the L-cysteine concentration was fixed at $5 \mathrm{mM}$ (for experiments with L-Cys-AMS) or $3 \mathrm{mM}$ (for experiments with other inhibitors) and the ATP concentration was varied between 0-5 $\mathrm{mM}$ (for experiments with L-Cys-AMS) or 0-3 $\mathrm{mM}$ (for experiments with other inhibitors). The $K_{\mathrm{m}}$ values used in the equation to determine $K_{\mathrm{i}}$ values were as previously reported: $K_{\mathrm{m}}{ }^{\mathrm{ATP}}=360 \mu \mathrm{M}, K_{\mathrm{m}}{ }^{\mathrm{L}-\mathrm{Cys}}=650 \mu \mathrm{M}^{20}$ All data sets were fitted using Kaleidagraph $^{\mathrm{TM}}$ software.
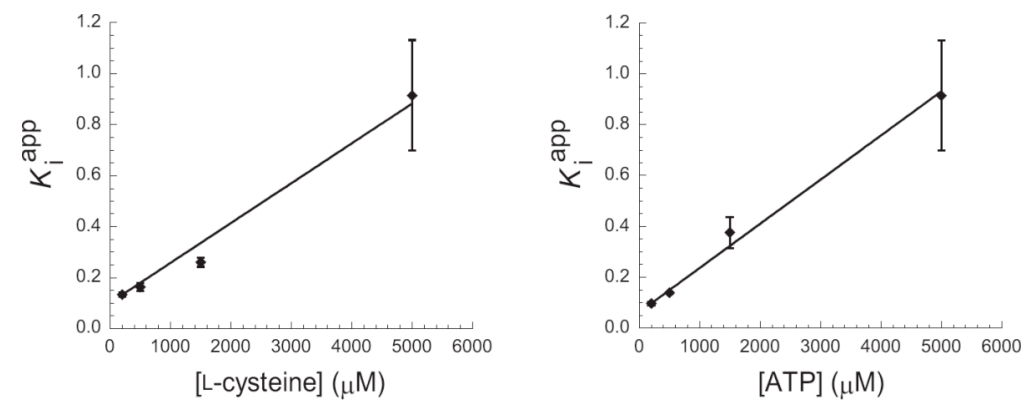

Figure S7. $K_{\mathrm{i}}$ determination for Cys-AMS (1a) with varied cysteine (left) and ATP (right) concentrations. $K_{\mathrm{i}}^{\text {Cys }}=0.101 \pm 0.005 \mu \mathrm{M} ; K_{\mathrm{i}}^{\mathrm{ATP}}=0.062 \pm 0.002 \mu \mathrm{M}$.

\footnotetext{
${ }^{24}$ Copeland, R. A. In Enzymes: A practical introduction to structure, mechanism, and data analysis; 2nd ed.; Wiley-
} VCH, Inc. Publications: New York, 2000, pp 305-317. 

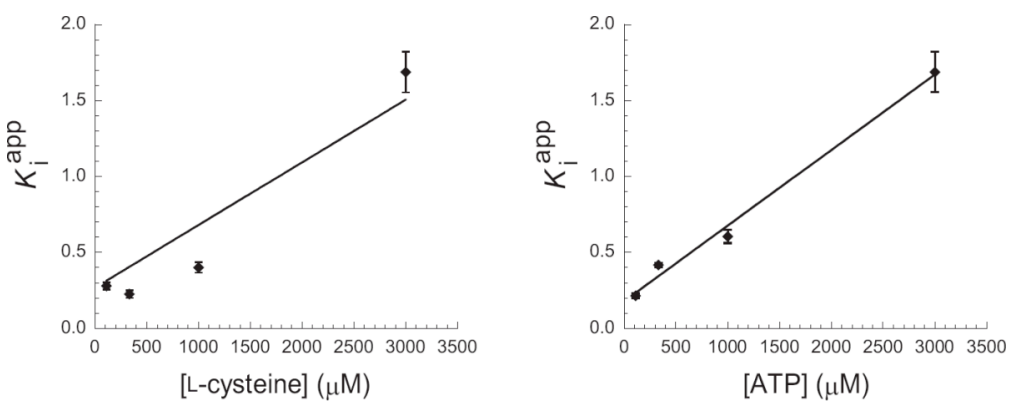

Figure S8. $K_{\mathrm{i}}$ determination for macrocycle 2a with varied cysteine (left) and ATP (right) concentrations. $K_{\mathrm{i}}^{\text {Cys }}=0.27 \pm 0.03 \mu \mathrm{M} ; K_{\mathrm{i}}^{\mathrm{ATP}}=0.179 \pm 0.005 \mu \mathrm{M}$.
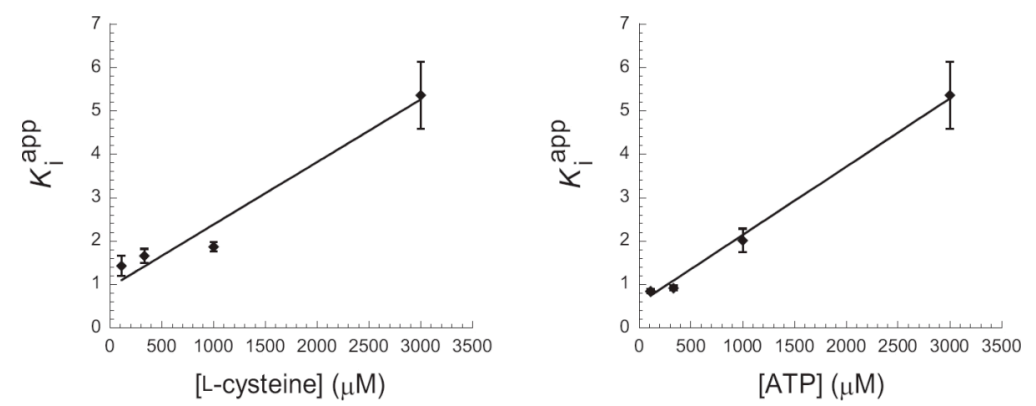

Figure S9. $K_{\mathrm{i}}$ determination for macrocycle $2 \mathrm{~b}$ with varied cysteine (left) and ATP (right) concentrations. $K_{\mathrm{i}}^{\text {Cys }}=0.92 \pm 0.06 \mu \mathrm{M} ; K_{\mathrm{i}}^{\text {ATP }}=0.57 \pm 0.01 \mu \mathrm{M}$.

\section{IN VITRO TRANSLATION ASSAY}

In vitro translation of luciferase was carried out using a $\mathrm{T} 7$ coupled rabbit reticulocyte system (Promega Cat. No. L4610). Rabbit reticulocyte lysate extracts were prepared according to the manufacturer's instructions, which included DNA addition. Translation reactions were carried out at $37^{\circ} \mathrm{C}$ for $1.5 \mathrm{~h}$ in low volume 384-well plates (Corning Inc.) by adding $9 \mu \mathrm{L}$ extract and $4 \mu \mathrm{L}$ of the inhibitor (1a, $\mathbf{1 b}, \mathbf{2 a}$, or $\mathbf{2 b}$; final concentration $250-0.01 \mu \mathrm{M})$ ) in nuclease-free water. Luminescence was measured in opaque 96-well plates (Corning Inc.) by adding the luciferin reagent to a $2 \mu \mathrm{L}$ aliquot and recording luminescence with a luminometer. 
A)

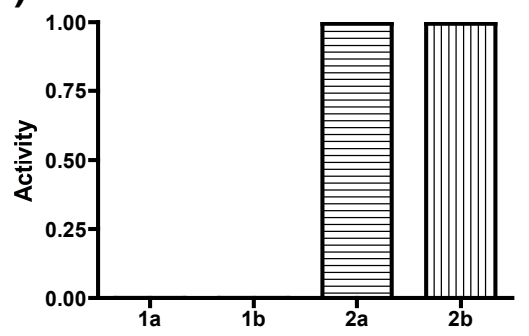

D)

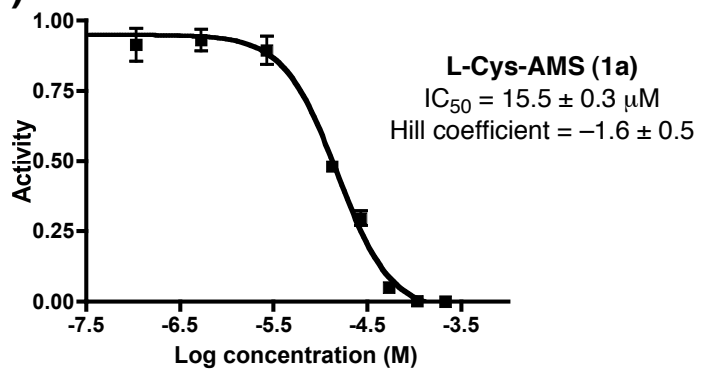

B)

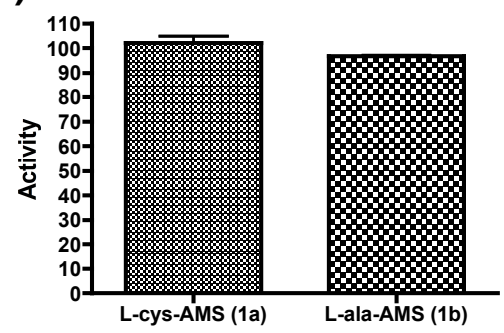

E)

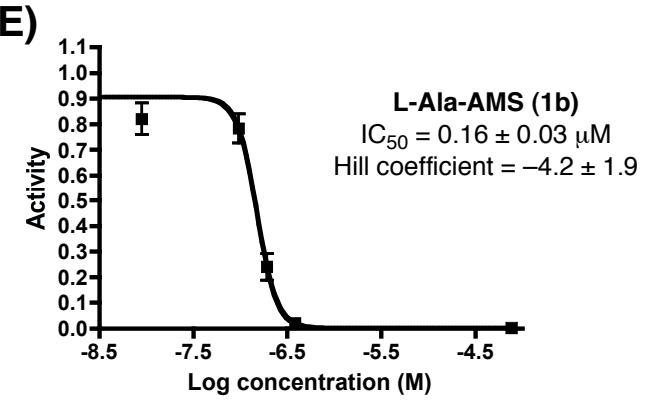

Figure S10. Inhibition of luciferase in vitro translation. (A, C) L-Cysteyl-AMS (1a) and L-alanyl-AMS (1b) strongly inhibit in vitro translation when tested at $250 \mu \mathrm{M}$ concentration, but macrocycles $\mathbf{2 a}$ and $\mathbf{2 b}$ do not. (B, C) Although luciferase is structurally and mechanistically related to NRP amino acid adenylation enzymes, neither cysteyl-AMS nor alanyl-AMS inhibits luciferase activity directly as evidenced by their lack of activity when added post-translationally at $250 \mu \mathrm{M}$ concentration. (D) Dose response curve for inhibition of in vitro translation by L-cysteyl-AMS (1a) to determine $I_{50}$ value and Hill coefficient. (E) Dose response curve for inhibition of in vitro translation by L-alanyl-AMS (1a) to determine $\mathrm{IC}_{50}$ value and Hill coefficient.

\section{J. NMR SPECTRA FOR MACROCYCLES 2a-e}

1. Macrocycle 2a $-{ }^{1} \mathrm{H}-\mathrm{NMR} \quad \mathrm{S} 35$

2. Macrocycle $\mathbf{2 a}-{ }^{13} \mathrm{C}-\mathrm{NMR} \quad \mathrm{S} 36$

3. Macrocycle $\mathbf{2 b}-{ }^{1} \mathrm{H}-\mathrm{NMR} \quad \mathrm{S} 37$

4. Macrocycle $\mathbf{2 b}-{ }^{13} \mathrm{C}-\mathrm{NMR} \quad \mathrm{S} 38$

5. Macrocycle $\mathbf{2 c}-{ }^{1} \mathrm{H}-\mathrm{NMR} \quad \mathrm{S} 39$

6. Macrocycle $2 \mathrm{c}-{ }^{13} \mathrm{C}-\mathrm{NMR} \quad \mathrm{S} 40$

7. Macrocycle 2d $-{ }^{1} \mathrm{H}-\mathrm{NMR} \quad \mathrm{S} 41$

8. Macrocycle 2d $-{ }^{13} \mathrm{C}-\mathrm{NMR} \quad \mathrm{S} 42$

9. Macrocycle 2e $-{ }^{1} \mathrm{H}-\mathrm{NMR} \quad \mathrm{S} 43$

10. Macrocycle $2 \mathbf{e}-{ }^{13} \mathrm{C}-\mathrm{NMR} \quad \mathrm{S} 44$ 


\section{${ }^{1}$ H-NMR SPECTRUM FOR MACROCYCLE 2a}

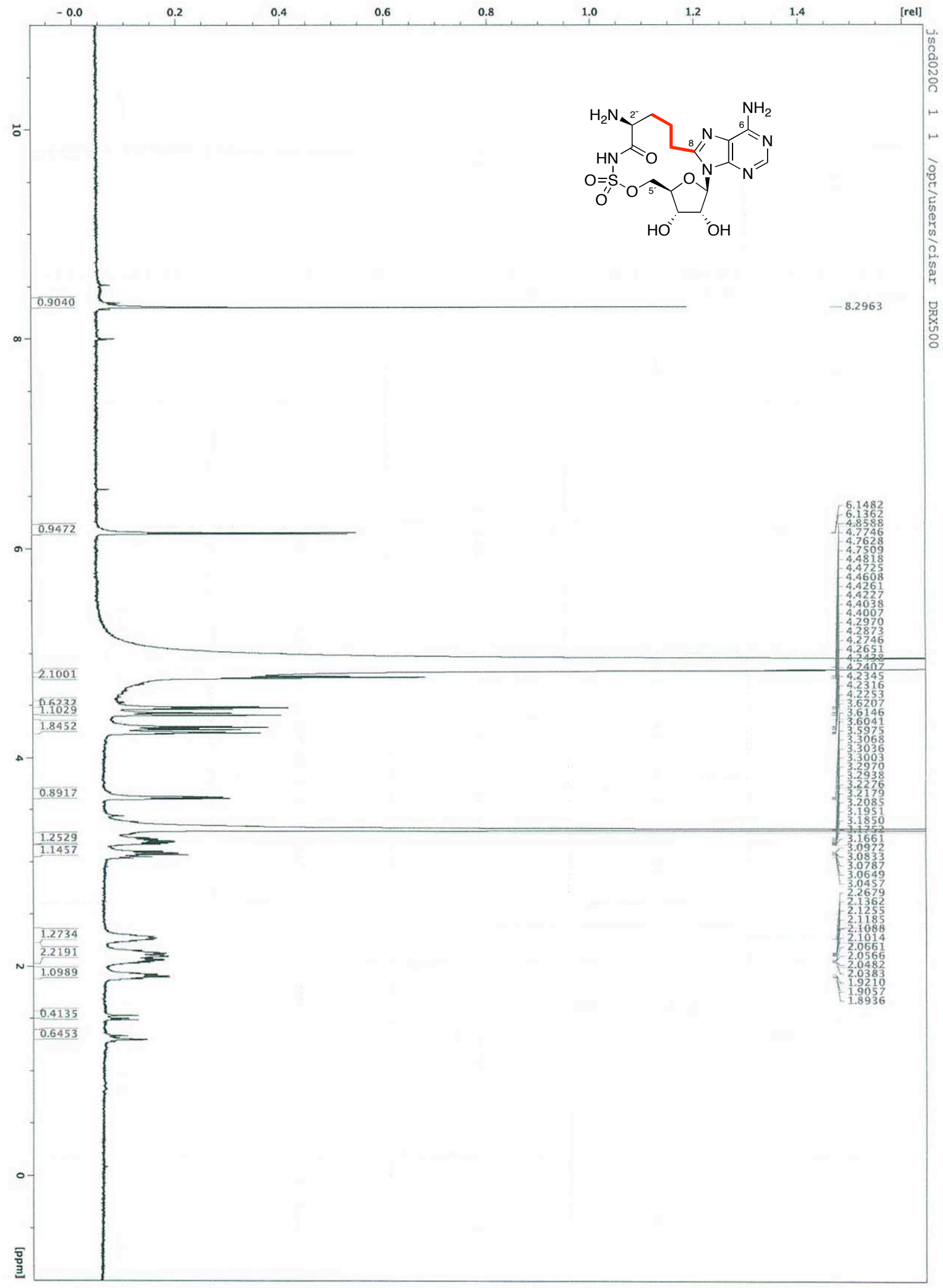




\section{${ }^{13}$ C-NMR SPECTRUM FOR MACROCYCLE $2 \mathrm{a}$}

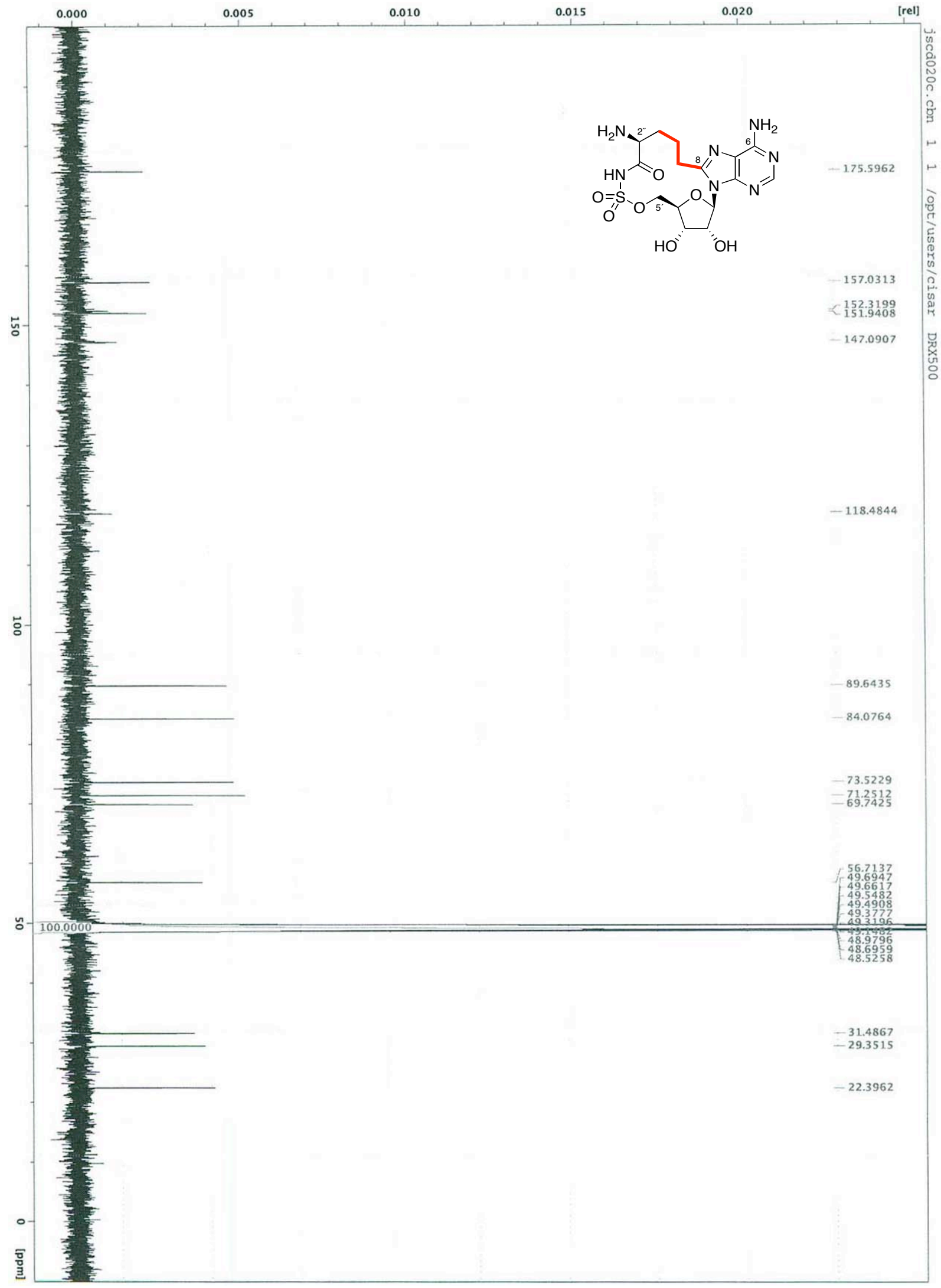




\section{${ }^{1}$ H-NMR SPECTRUM FOR MACROCYCLE 2b}

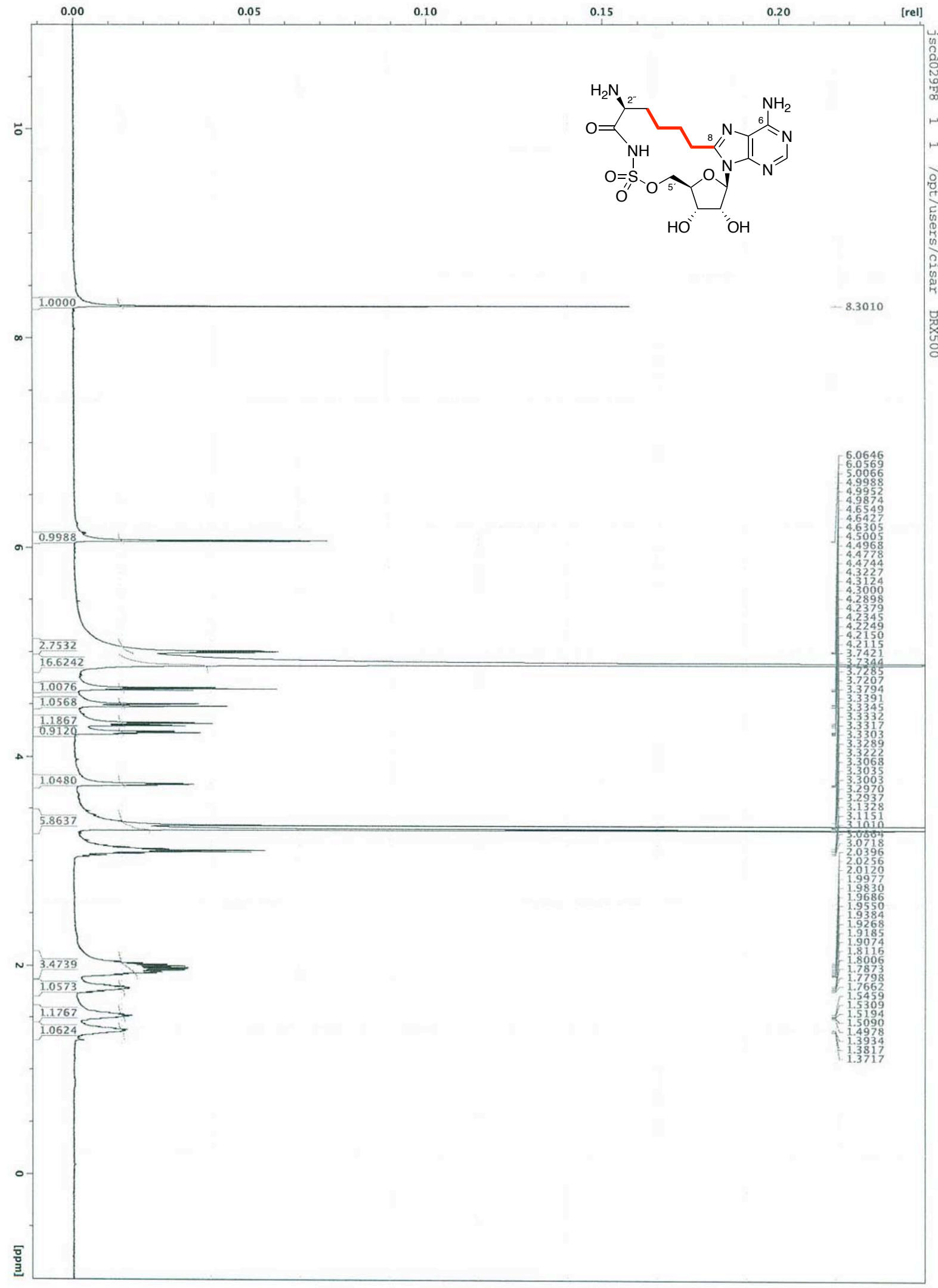




\section{${ }^{13}$ C-NMR SPECTRUM FOR MACROCYCLE $2 \mathrm{~b}$}

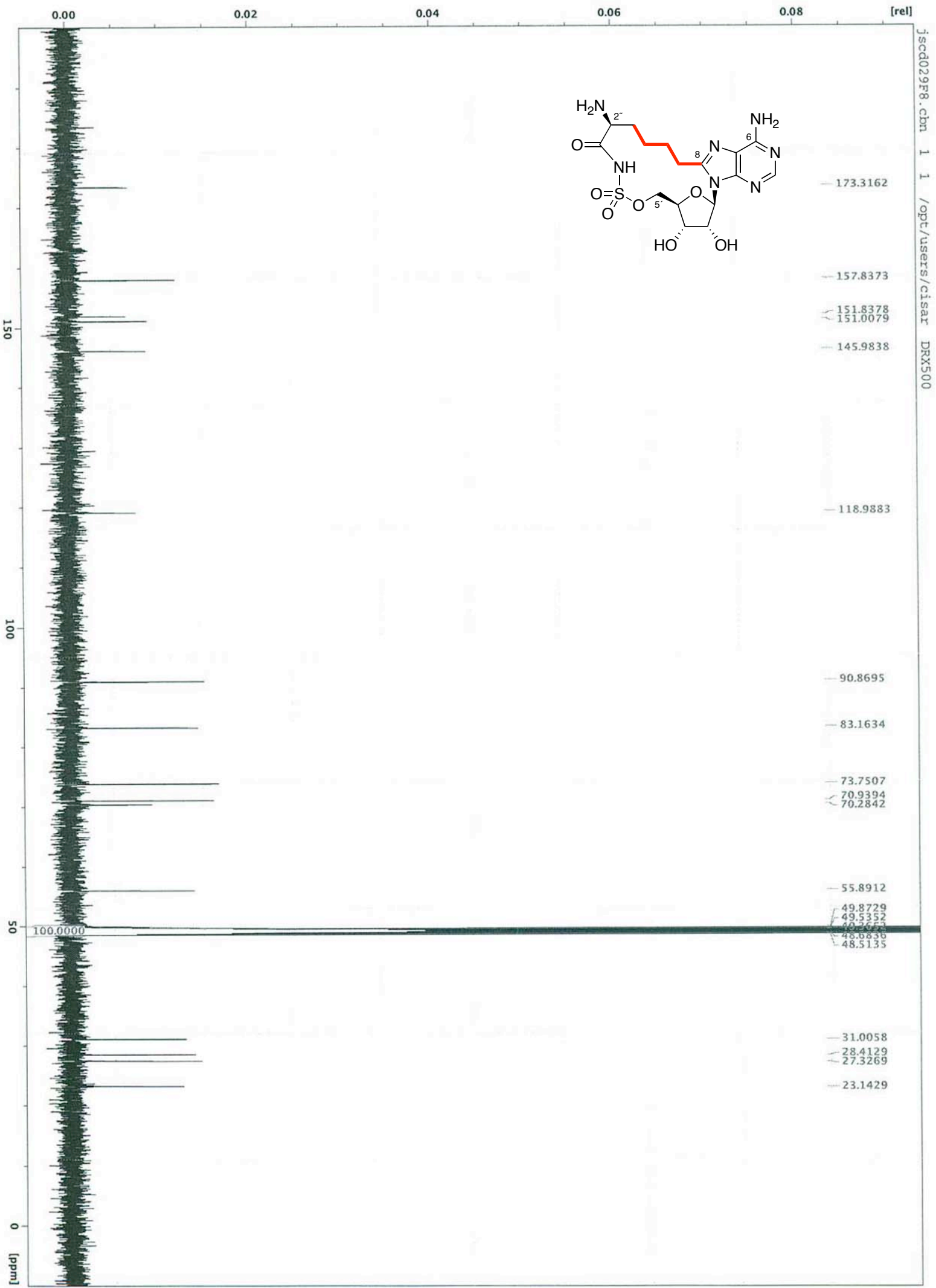




\section{${ }^{1}$ H-NMR SPECTRUM FOR MACROCYCLE 2C}

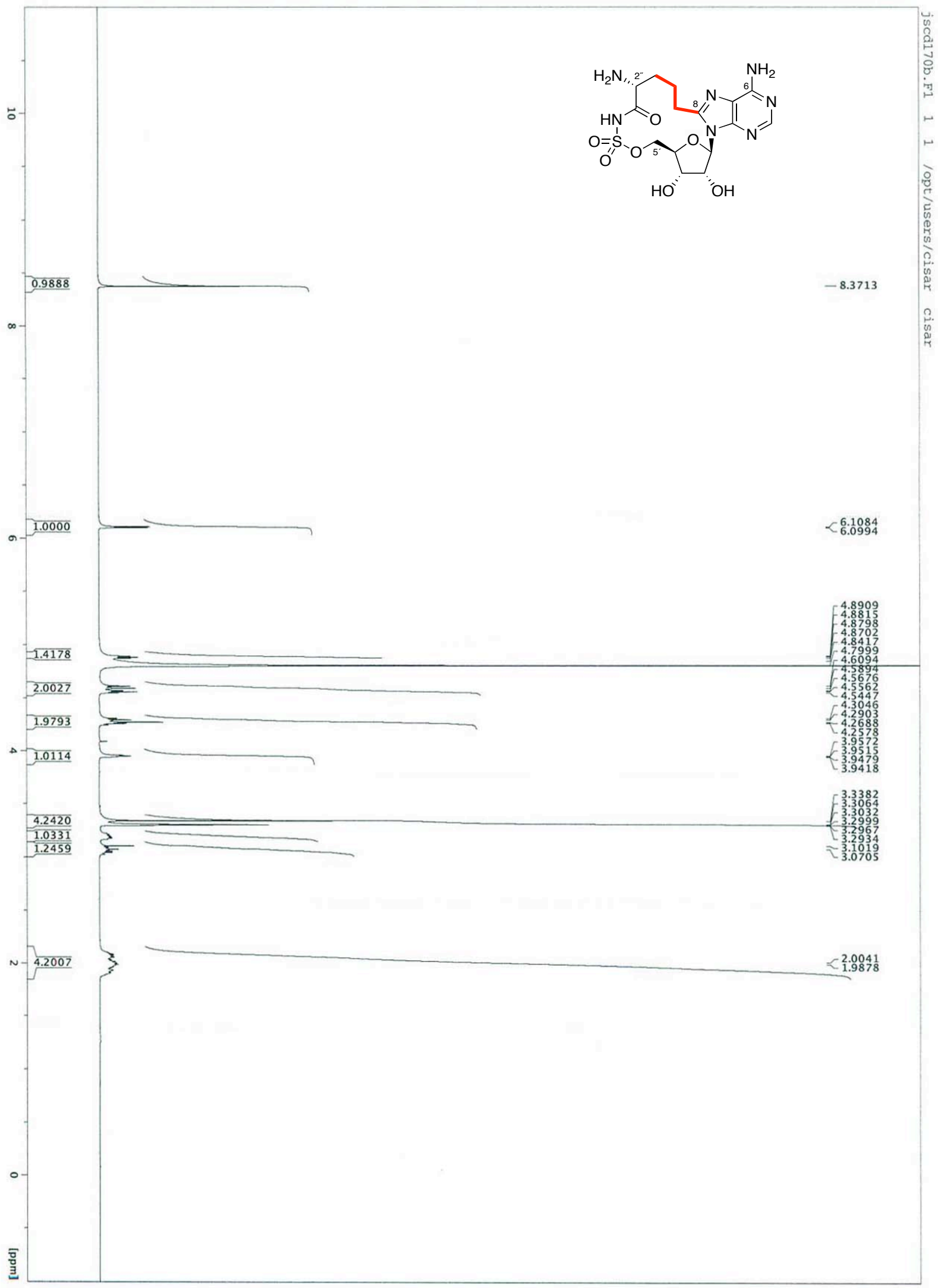




\section{${ }^{13}$ C-NMR SPECTRUM FOR MACROCYCLE 2c}

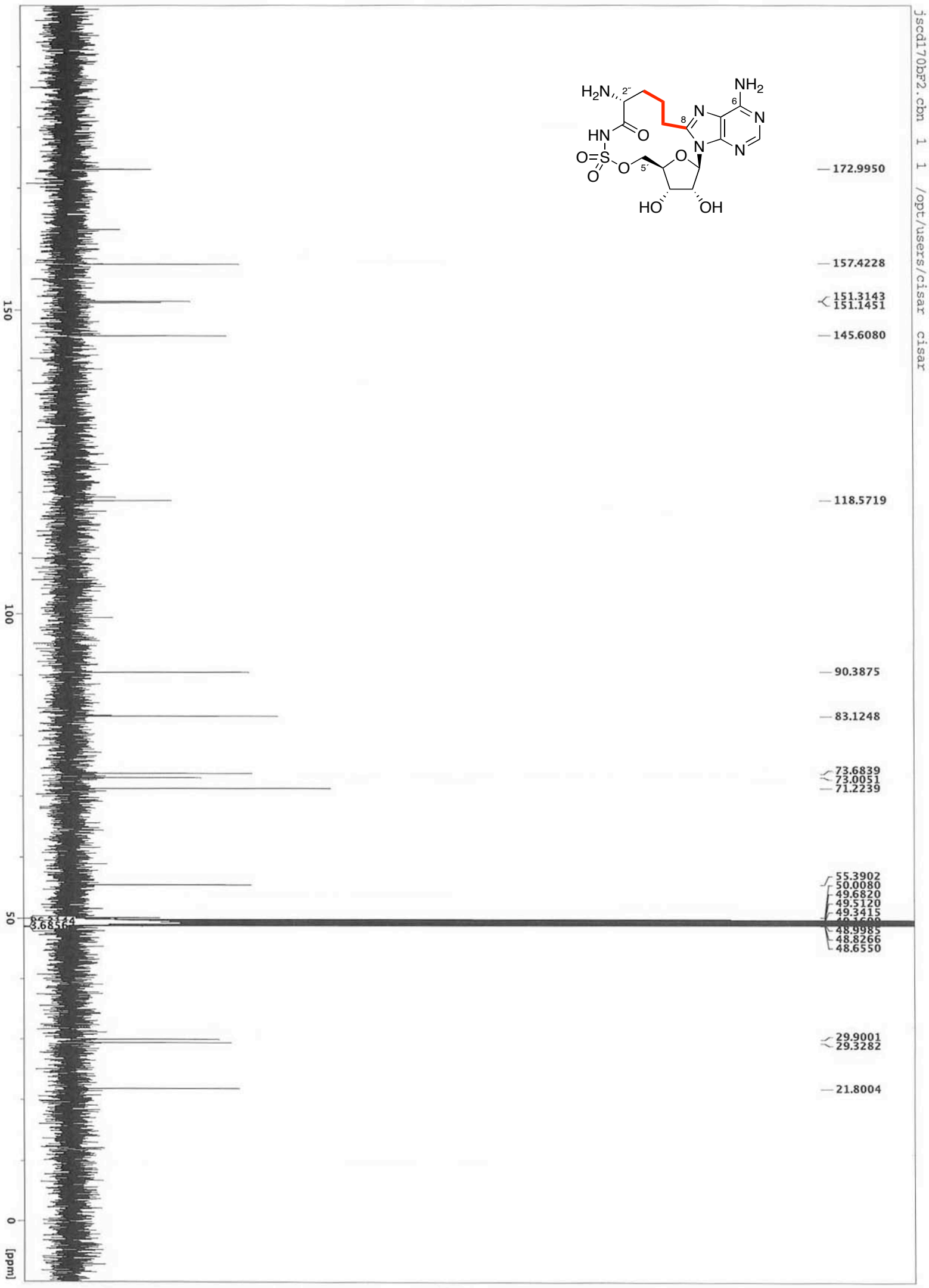




\section{${ }^{1}$ H-NMR SPECTRUM FOR MACROCYCLE 2d}

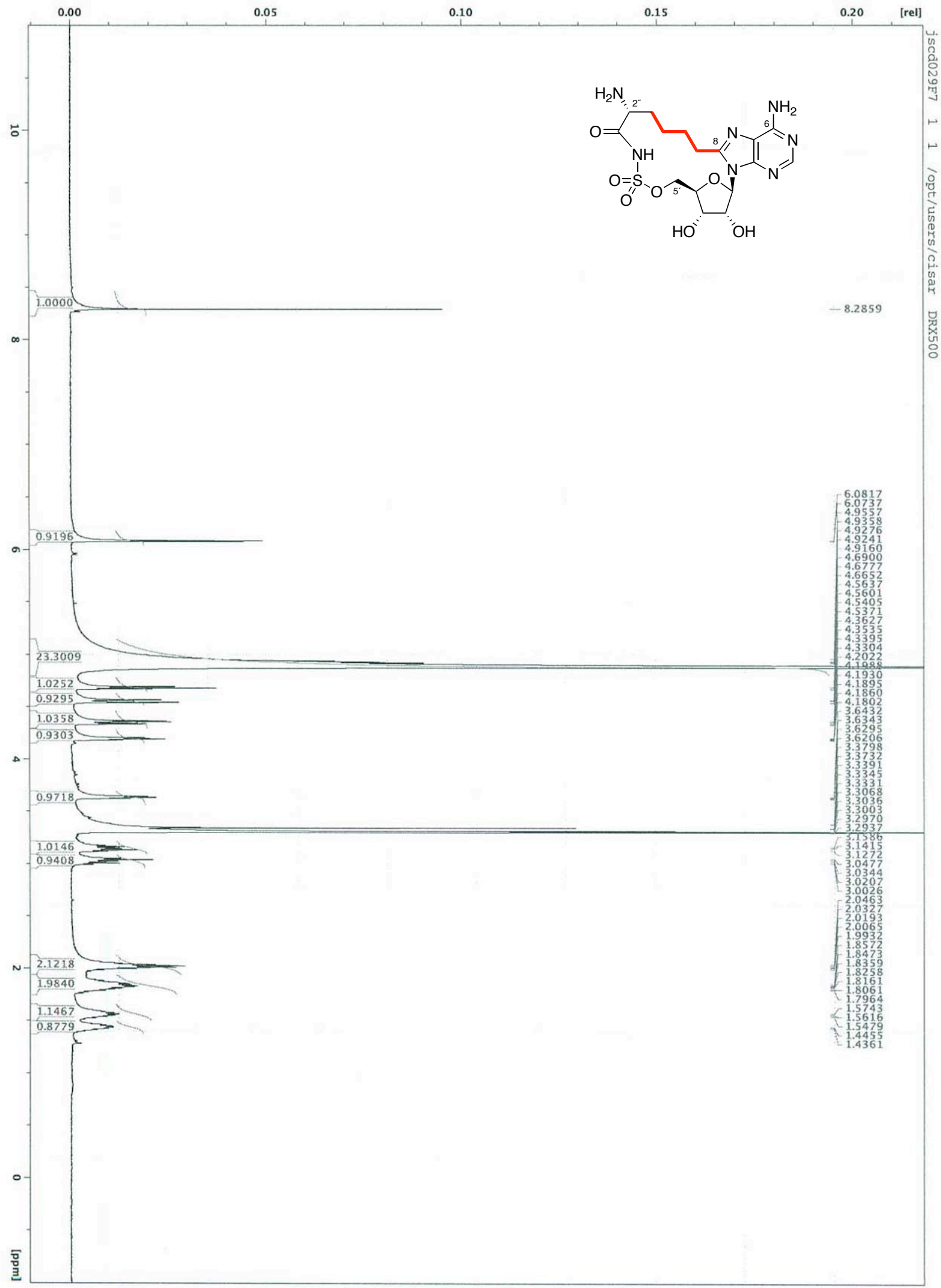




\section{${ }^{13}$ C-NMR SPECTRUM FOR MACROCYCLE 2d}

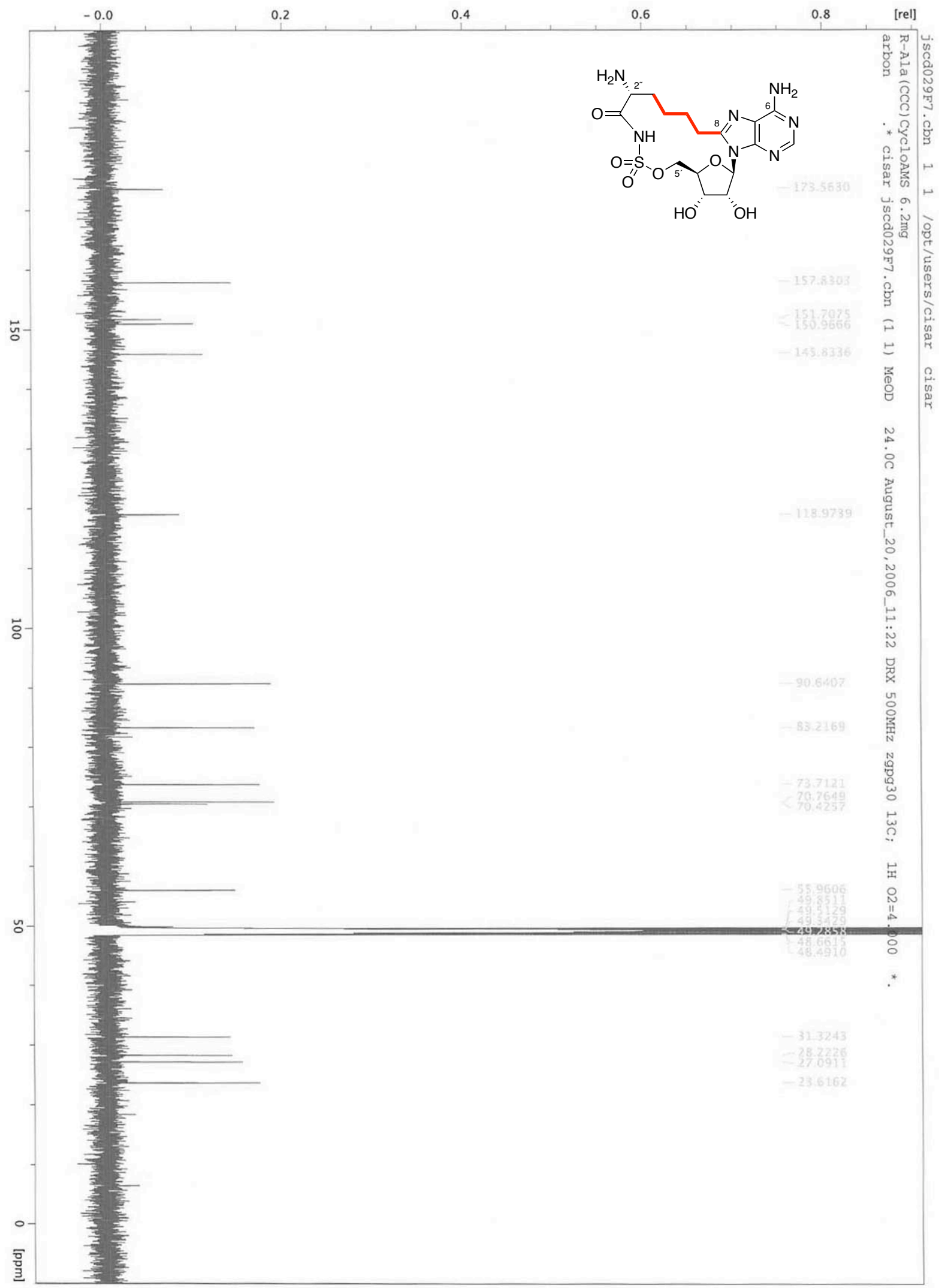




\section{${ }^{1}$ H-NMR SPECTRUM FOR MACROCYCLE $2 \mathrm{e}$}

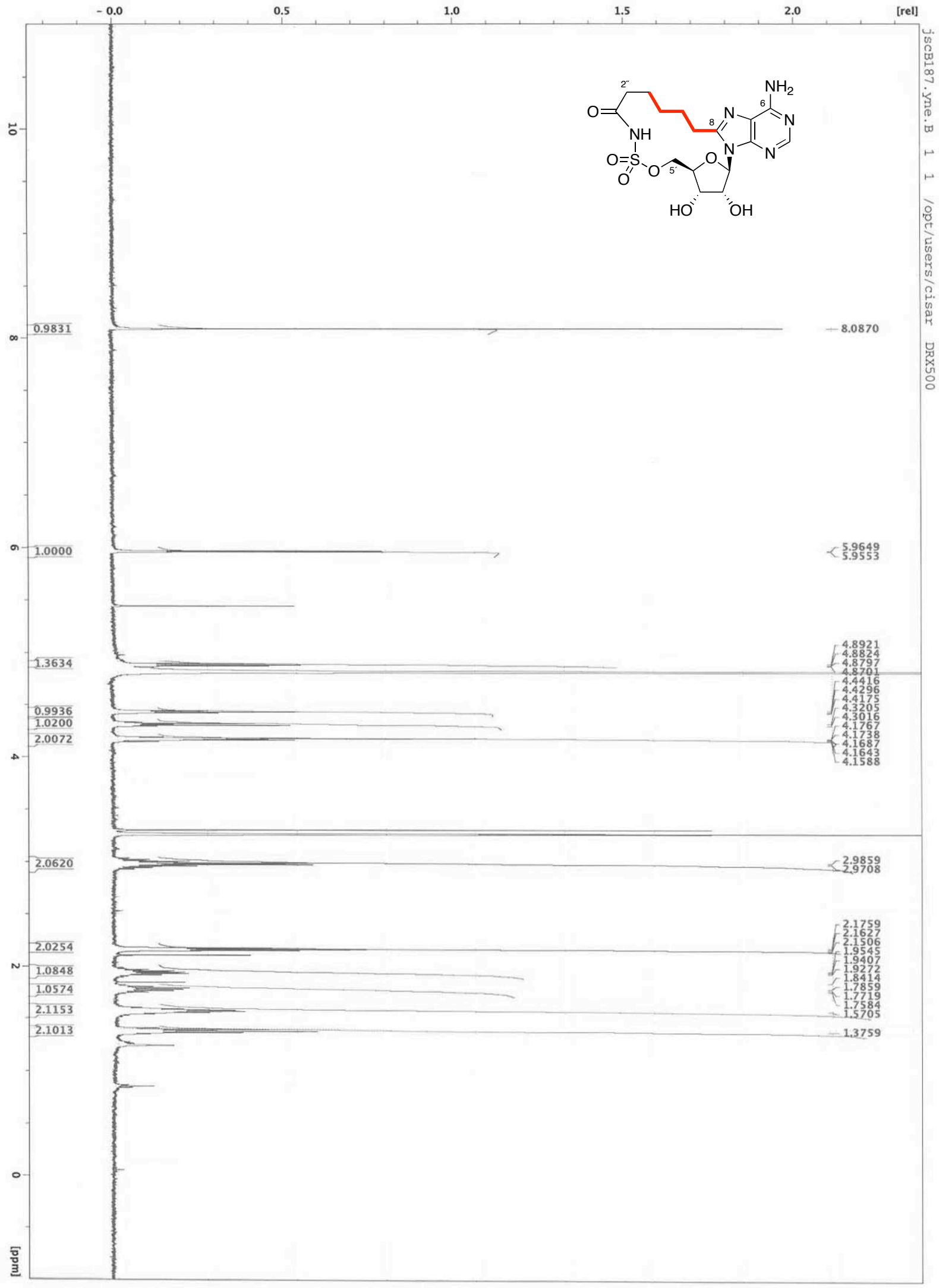




\section{${ }^{13}$ C-NMR SPECTRUM FOR MACROCYCLE 2e}

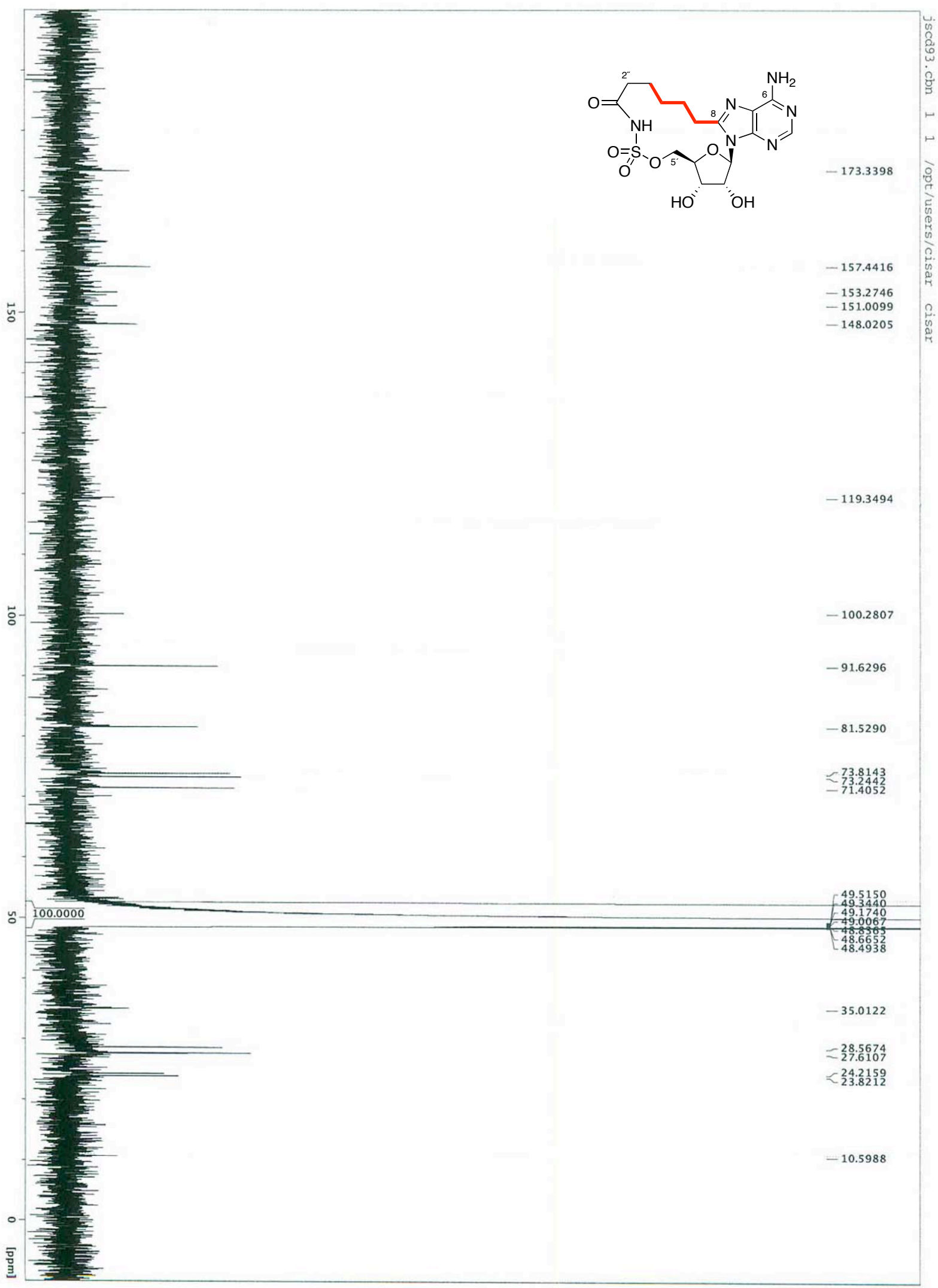

